TRANSACTIONS OF THE

AMERICAN MATHEMATICAL SOCIETY

Volume 361, Number 1, January 2009, Pages 407-452

S 0002-9947(08)04581-9

Article electronically published on July 24, 2008

\title{
REPARAMETRIZATION INVARIANT NORMS
}

\author{
P. FROSINI AND C. LANDI
}

\begin{abstract}
This paper explores the concept of reparametrization invariant norm (RPI-norm) for $C^{1}$-functions that vanish at $-\infty$ and whose derivative has compact support, such as $C_{c}^{1}$-functions. An RPI-norm is any norm invariant under composition with orientation-preserving diffeomorphisms. The $L_{\infty}$-norm and the total variation norm are well-known instances of RPI-norms. We prove the existence of an infinite family of RPI-norms, called standard RPInorms, for which we exhibit both an integral and a discrete characterization. Our main result states that for every piecewise monotone function $\varphi$ in $C_{c}^{1}(\mathbb{R})$ the standard RPI-norms of $\varphi$ allow us to compute the value of any other RPInorm of $\varphi$. This is proved using the standard RPI-norms to reconstruct the function $\varphi$ up to reparametrization, sign and an arbitrarily small error with respect to the total variation norm.
\end{abstract}

\section{INTRODUCTION}

In recent papers the natural pseudo-distance $\sigma$ between manifolds endowed with regular real functions has been studied as a tool for comparing the shape of manifolds (cf. [4, 5, 6]). Each shape is represented by pairs $(\mathcal{M}, \varphi)$, where $\mathcal{M}$ is a connected manifold and $\varphi$ is a real function defined on it (both $\mathcal{M}$ and $\varphi$ are supposed to be sufficiently regular). In this approach, the main idea is to compare two diffeomorphic manifolds by measuring the global change of the real functions they are endowed with when the manifolds are deformed into each other: $\sigma((\mathcal{M}, \varphi),(\mathcal{N}, \psi))=\inf _{h} \sup _{p \in \mathcal{M}}|\varphi(p)-\psi \circ h(p)|$, where $h$ varies among all the diffeomorphisms between $\mathcal{M}$ and $\mathcal{N}$. We observe that $\sigma$ is a Fréchet-like distance (cf., e.g., 7]). Moreover, this line of research is strongly related to the extensive study currently being carried out on parametrization-independent shape comparison in Pattern Recognition (cf, e.g., [10]).

The definition of natural pseudo-distance between the pairs $\left(\mathcal{M}, \varphi_{1}\right),\left(\mathcal{M}, \varphi_{2}\right)$ can be reformulated as the value $\inf _{h \in D} F\left(\varphi_{1}-\varphi_{2} \circ h\right)$, where $D$ denotes the set of all diffeomorphisms from $\mathcal{M}$ to $\mathcal{M}$ and $F$ is the norm that takes each (sufficiently regular) function $\bar{\varphi}: \mathcal{M} \rightarrow \mathbb{R}$ to the number $\|\bar{\varphi}\|_{\infty}=\max _{P \in \mathcal{M}}|\bar{\varphi}(P)|$. In order that $\inf _{h \in D} F\left(\varphi_{1}-\varphi_{2} \circ h\right)$ is a pseudo-distance, the key property of the functional $F$ is that $F$ is a norm and $F(\bar{\varphi} \circ h)=F(\bar{\varphi})$ for every $\bar{\varphi}: \mathcal{M} \rightarrow \mathbb{R}$ and every $h \in D$. In other words, the point is that $F$ is a reparametrization invariant norm. Choosing a different reparametrization invariant norm would allow us to obtain a different pseudo-distance.

Received by the editors March 21, 2007.

2000 Mathematics Subject Classification. Primary 46E10, 46B20.

Key words and phrases. Reparametrization invariant norm, standard reparametrization invariant norm.

(C)2008 American Mathematical Society Reverts to public domain 28 years from publication 
From this simple observation some natural questions arise. Are there other reparametrization invariant norms on the vector space $V$ of all (sufficiently regular) real functions defined on $\mathcal{M}$ ? What are their properties? Are they induced by inner products? What kind of information do they contain? Is it possible to reconstruct a function $\varphi$ using the values taken at $\varphi$ by the norms in the set $R P I_{V}$ of all reparametrization invariant norms on $V$, or by the norms in a suitable subset of $R P I_{V}$ ?

It is clear that progress in this line of research requires answers to these questions. This paper is a first step in this direction, studying what happens in the simplest case, i.e. $\mathcal{M}=\mathbb{R}$, when the considered reparametrizations are orientationpreserving.

We conclude this introduction by recalling that invariance under reparametrization appears to be relevant to several fields of research. Among these, two examples are Statistics (cf., e.g., the Kolmogorov-Smirnov Test) and the Theory of Interpolation Spaces (e.g., with reference to the K-Method). Nevertheless, to the best of our knowledge, little is known about these norms, with the exceptions of translation invariant norms (see, e.g., 9]) and dilation invariant norms (see, e.g., 11]).

1.1. The main ideas in this paper. This paper studies the reparametrization invariant norms that can be defined on a suitable set of regular functions $\varphi$ from $\mathbb{R}$ to $\mathbb{R}$. The norms $\max |\varphi|, \max \varphi-\min \varphi$, the total variation $V_{\varphi}$ of $\varphi$, and the function $\sqrt{\max |\varphi|^{2}+V_{\varphi}^{2}}$ are simple examples of reparametrization invariant norms, assuming that the derivative of $\varphi$ has compact support, and that $\varphi$ vanishes at $-\infty$. Although many norms exist that are not reparametrization invariant, such as the $L_{p}$-norms on $C_{c}^{1}(\mathbb{R})$ for $p<+\infty$, there exists an infinite number of RPInorms, since each linear combination with positive coefficients of reparametrization invariant norms is obviously a reparametrization invariant norm. In the set of all the RPI-norms, we have succeeded in detecting a particular subset of norms, which we call standard reparametrization invariant ( $R P I-)$ norms, such that

(1) if the $C^{1}$-function $\varphi$ has compact support and is piecewise monotone, then knowledge of all the standard RPI-norms of $\varphi$ allows us to reconstruct $\varphi$ up to reparametrization, with an arbitrarily small error $\varepsilon$ with respect to the total variation norm;

(2) as a consequence of the previous property, any other RPI-norm of such a function $\varphi$ is completely determined by the values taken on $\varphi$ by the standard RPI-norms.

Therefore, we have focused our research on these norms.

The main idea of this paper originates from the following classical definition of the total variation $V_{\varphi}$ for a regular function $\varphi: \mathbb{R} \rightarrow \mathbb{R}$ (see, e.g., [1]):

$$
V_{\varphi}=\sup _{\psi \in \Psi}\left|\int_{-\infty}^{+\infty} \varphi(t) \cdot \frac{d \psi}{d t}(t) d t\right|=\sup _{\psi \in \Psi}\left|\int_{-\infty}^{+\infty} \varphi(-t) \cdot \frac{d \psi}{d t}(t) d t\right|,
$$

where $\Psi$ is the set of all (sufficiently regular) functions $\psi$ from $\mathbb{R}$ to $\mathbb{R}$ with $|\psi| \leq 1$. We observe that if we substitute $\Psi$ with any subset $\hat{\Psi}$ of $\Psi$ that is closed with respect to reparametrization, then other reparametrization invariant norms can be obtained (though, in this case, the two suprema in the previous formula may be different). The closure with respect to reparametrization means that if $\hat{\psi} \in \hat{\Psi}$, then $\hat{\psi} \circ h \in \hat{\Psi}$ for every orientation-preserving diffeomorphism $h: \mathbb{R} \rightarrow \mathbb{R}$. 
In order to apply our idea, we first choose a functional space. Many different choices are possible. As a trade-off between generality and simplicity we have chosen the space $A S^{1}(\mathbb{R})$ of all almost sigmoidal $C^{1}$-functions. Roughly speaking, this space could be defined as the space of all $C^{1}$-functions $\psi: \mathbb{R} \rightarrow \mathbb{R}$ that "behave as a sigmoid outside a sufficiently large compact" (see Section 2, Definition 2.1). This choice is not very restrictive, since $A S^{1}(\mathbb{R})$ contains all the $C^{1}$-functions with compact support.

By defining $[\psi]$ as the set containing $\psi \in A S^{1}(\mathbb{R})$ and all its reparametrizations $\psi \circ h$, and by setting

$$
\|\varphi\|_{[\psi]}=\sup _{\hat{\psi} \in[\psi]}\left|\int_{-\infty}^{+\infty} \varphi(-t) \cdot \frac{d \hat{\psi}}{d t}(t) d t\right|
$$

we obtain a reparametrization invariant norm on $A S^{1}(\mathbb{R})$. The norms obtained in this way are precisely the standard reparametrization invariant norms, verifying the properties described in the previous statements (1) and (2) (Theorem 5.8). The reason for using $\varphi(-t)$ instead of $\varphi(t)$ inside the integral is that this choice allows us to obtain the equality $\|\varphi\|_{[\psi]}=\|\psi\|_{[\varphi]}$, thanks to the fact that our functions belong to $A S^{1}(\mathbb{R})$. Incidentally, this also motivated the choice of $A S^{1}(\mathbb{R})$ as the functional space to use.

We could proceed analogously by taking, in place of $[\psi]$, a general set $\hat{\Psi}$ closed with respect to reparametrization, and obtain a reparametrization invariant norm $\|\varphi\|_{\hat{\Psi}}$, but the case $\hat{\Psi}=[\psi]$ is the most interesting one, since $\|\varphi\|_{\hat{\Psi}}$ can easily be expressed as a supremum of standard reparametrization invariant norms.

In order to get the main results of this paper some technicalities will be necessary. In particular, a key role will be played by the Bounding Lemma 2.9. asserting that, after normalization, every reparametrization invariant norm $\|\varphi\|$ is upper bounded by the total variation $V_{\varphi}$ and lower bounded by the value $\lim _{t \rightarrow+\infty}|\varphi(t)|$. A stronger Bounding Lemma will be proved for $C_{c}^{1}(\mathbb{R})$. It asserts that, after normalization, every reparametrization invariant norm $\|\varphi\|$ of a function $\varphi$, having compact support, is upper bounded by half the total variation $V_{\varphi}$ and lower bounded by the value $\max |\varphi(t)|$. The proof of these key results will require some computations and a preliminary study of the general properties of reparametrization invariant norms, that will be carried out in Section2. In particular, we shall examine the role played by two particular functions, called $S$ and $\Lambda$. Moreover, in the same section we shall prove the stability of RPI-norms with respect to small perturbations in $C^{1}$, and the interesting fact that no inner product can induce an RPI-norm.

The definition of standard reparametrization invariant norm will be introduced in Section 3, together with some examples and basic properties. However, in order to proceed further, we shall have to represent standard reparametrization norms in a simpler way. We know that an alternative definition exists for the total variation, saying that $V_{\varphi}$ equals the value $\sup _{n} \sup _{\tau_{0} \leq \ldots \leq \tau_{i} \leq \ldots \leq \tau_{n}} \sum_{i=0}^{n-1}\left|\varphi\left(\tau_{i+1}\right)-\varphi\left(\tau_{i}\right)\right|$, for $\varphi \in A S^{1}(\mathbb{R})$. In Section 4 some computations will be necessary to make available a similar representation also for standard reparametrization invariant norms (Theorem 4.16). This new kind of representation will be used to prove the fundamental results in this paper, i.e. the possibility of reconstructing piecewise monotone functions with compact support up to reparametrization and sign, by means of standard 
reparametrization invariant norms, and the dependence of reparametrization invariant norms on standard reparametrization invariant norms (Section 5). Section 6 will conclude this paper by illustrating some open problems.

Addendum: During the proof-reading of this paper, it has come to our knowledge that, in the case of real functions defined on a compact $n$-manifold with $n \geq 2$, reparametrization invariant norms have been studied in [12. In particular, the author proves that if $N$ is a $\operatorname{Diff}_{0}^{k}$-invariant semi-norm on $C^{k}(X)(k \geq 1)$, where $X$ is a compact, connected and without boundary manifold of dimension at least 2 , one verifies that $N(f)=\nu(\inf f, \sup f)$, where $\nu$ is a semi-norm on $\mathbb{R}^{2}$ invariant by the symmetry $(a, b) \rightarrow(b, a)$ and $\nu(a, b)$ is an increasing function of the interval $[a, b]($ for $a \leq b)$.

\section{REPARAMETRIZATION INVARIANT NORMS: DEFINITION AND GENERAL PROPERTIES}

2.1. Some notation and basic definitions. In this paper the symbols $C^{1}(\mathbb{R})$ and $C_{c}^{1}(\mathbb{R})$ will represent the set of all one-time continuously differentiable functions from $\mathbb{R}$ to $\mathbb{R}$, and the set of all functions in $C^{1}(\mathbb{R})$ that have compact support, respectively. The symbol $D_{+}^{1}(\mathbb{R})$ will represent the set of all orientation-preserving $C^{1}$-diffeomorphisms from $\mathbb{R}$ to $\mathbb{R}$.

We shall say that a function $f$ is increasing (strictly increasing) if $t<t^{\prime}$ implies $f(t) \leq f\left(t^{\prime}\right)\left(f(t)<f\left(t^{\prime}\right)\right)$, and decreasing (strictly decreasing) if $t<t^{\prime}$ implies $f(t) \geq f\left(t^{\prime}\right)\left(f(t)>f\left(t^{\prime}\right)\right)$. A function will be called monotone if it is either decreasing or increasing, and strictly monotone if it is either strictly increasing or strictly decreasing.

A number will be said to be positive when it is strictly greater than zero. The set of positive natural numbers will be denoted by $\mathbb{N}^{+}$.

First of all, let us introduce the functional space we shall work in.

Definition 2.1. Let us consider the set of all $C^{1}$-functions $\varphi: \mathbb{R} \rightarrow \mathbb{R}$ for which two real values $a, b$ exist such that:

- $\varphi(t)=0$ for every $t \in(-\infty, a]$;

- $\varphi(t)$ is constant in $[b,+\infty)$.

We shall denote this set by the symbol $A S^{1}(\mathbb{R})$ and call each function in $A S^{1}(\mathbb{R})$ an almost sigmoidal function of class $C^{1}$.

Examples of almost sigmoidal functions are shown in Figure 1.

Obviously, $C_{c}^{1}(\mathbb{R}) \subset A S^{1}(\mathbb{R})$ and every function in $A S^{1}(\mathbb{R})$ has bounded variation. We shall use the symbol $\mathbf{0}$ to denote the almost sigmoidal function that vanishes everywhere.

The ideas described in this paper can be extended to more general spaces, but we choose this setting in order to simplify our proofs from the technical point of view.

For every $\psi \in A S^{1}(\mathbb{R})$ we shall denote by $V^{+}\langle\psi\rangle(t)$ (resp. $\left.V^{-}\langle\psi\rangle(t)\right)$ the positive (resp. negative) variation of $\psi$, and by $V\langle\psi\rangle(t)$ the variation of $\psi$ :

$$
\begin{gathered}
V^{+}\langle\psi\rangle(t)=\int_{-\infty}^{t} \max \left\{\frac{d \psi}{d s}(s), 0\right\} d s, V^{-}\langle\psi\rangle(t)=\int_{-\infty}^{t} \max \left\{-\frac{d \psi}{d s}(s), 0\right\} d s \\
V\langle\psi\rangle(t)=V^{+}\langle\psi\rangle(t)+V^{-}\langle\psi\rangle(t)=\int_{-\infty}^{t}\left|\frac{d \psi}{d s}(s)\right| d s
\end{gathered}
$$




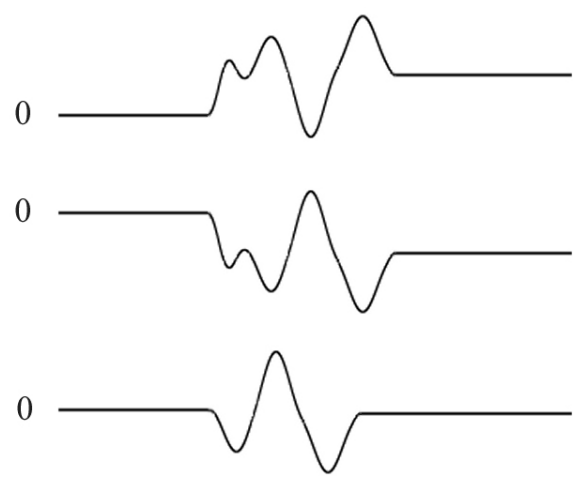

Figure 1. Three examples of almost sigmoidal functions.

Since $\psi \in C^{1}(\mathbb{R})$, the functions $V^{+}\langle\psi\rangle, V^{-}\langle\psi\rangle$, and $V\langle\psi\rangle$ are $C^{1}$. Moreover we shall denote by $V_{\psi}^{+}, V_{\psi}^{-}, V_{\psi}$ the total positive variation, the total negative variation and the total variation of $\psi$, respectively:

$$
\begin{gathered}
V_{\psi}^{+}=\int_{-\infty}^{+\infty} \max \left\{\frac{d \psi}{d s}(s), 0\right\} d s, V_{\psi}^{-}=\int_{-\infty}^{+\infty} \max \left\{-\frac{d \psi}{d s}(s), 0\right\} d s, \\
V_{\psi}=V_{\psi}^{+}+V_{\psi}^{-}=\int_{-\infty}^{+\infty}\left|\frac{d \psi}{d s}(s)\right| d s .
\end{gathered}
$$

We recall that $V^{+}\langle\psi\rangle(t)$ and $V^{-}\langle\psi\rangle(t)$ are non-negative increasing functions whose difference is exactly $\psi$. Observe that $V_{\psi}^{+}-V_{\psi}^{-}=\lim _{t \rightarrow+\infty} \psi(t)$. Obviously, if $\psi \in C_{c}^{1}(\mathbb{R})$, then $V_{\psi}^{+}=V_{\psi}^{-}=\frac{1}{2} V_{\psi}$.

Definition 2.2. For any two functions $\varphi_{1}, \varphi_{2}: \mathbb{R} \rightarrow \mathbb{R}$, we say that $\varphi_{2}$ is obtained from $\varphi_{1}$ by a reparametrization (of class $C^{1}$ ) if an orientation-preserving diffeomorphism $h \in D_{+}^{1}(\mathbb{R})$ exists such that $\varphi_{2}=\varphi_{1} \circ h$. The diffeomorphism $h$ will be called a reparametrization. We denote by $\sim$ the equivalence relation defined by setting $\varphi_{2} \sim \varphi_{1}$ if and only if $\varphi_{2}$ is obtained from $\varphi_{1}$ by a reparametrization. The equivalence class of $\varphi_{1}$ in $A S^{1}(\mathbb{R})$ will be denoted by $\left[\varphi_{1}\right]$.

In this paper we study the norms that take equivalent functions to the same value (see Figure 2).

2.2. Reparametrization invariant norms. Now we give the main definition in this paper.

Definition 2.3. Let us consider the real vector space $A S^{1}(\mathbb{R})$. We say that a norm $\|\cdot\|: A S^{1}(\mathbb{R}) \rightarrow \mathbb{R}$ is invariant under reparametrization (or a reparametrization invariant norm) if it is constant over each equivalence class of $A S^{1}(\mathbb{R}) / \sim$.

In the following the reparametrization invariant norms will often be called RPInorms.

The norms $\max |\varphi|, \max \varphi-\min \varphi$ and the total variation $V_{\varphi}$ of $\varphi$ are simple examples of RPI-norms.

It is quite easy to see that an infinite number of RPI-norms exists. Indeed, it is trivial to prove that each linear combination with positive coefficients of a finite number of RPI-norms is still an RPI-norm. 


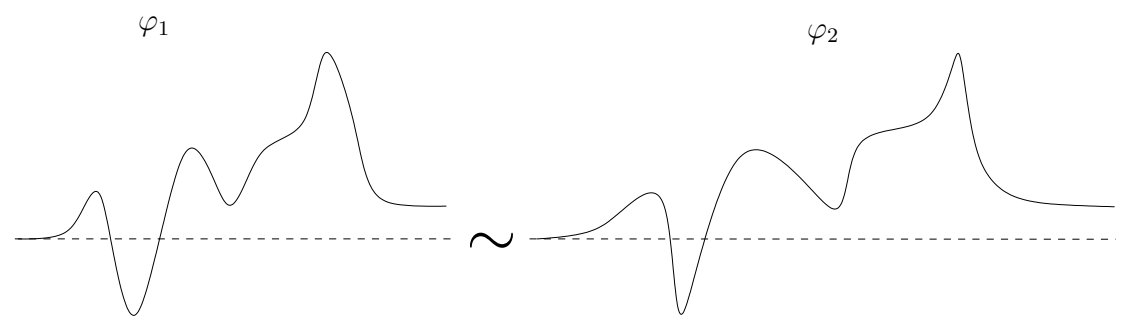

FiguRE 2. We are interested in studying the norms that take both the functions $\varphi_{1}$ and $\varphi_{2}$ to the same value, since $\varphi_{2}$ is obtained by composing $\varphi_{1}$ with an orientation-preserving $C^{1}$-diffeomorphism of $\mathbb{R}$.

Another simple method of obtaining an RPI-norm is to consider the sup of a set of RPI-norms, under the assumption that such a sup is finite at each point.

A third procedure consists in taking a norm $\|\cdot\|_{*}$ on $\mathbb{R}^{k}$ such that if $0 \leq x_{i} \leq y_{i}$ for $1 \leq i \leq k$, then $\left\|\left(x_{1}, \ldots, x_{k}\right)\right\|_{*} \leq\left\|\left(y_{1}, \ldots, y_{k}\right)\right\|_{*}$. This happens, e.g., when $\|\cdot\|_{*}$ is monotonic (cf. 2]). In this case it is easy to verify that, if we have $k$ RPI-norms $\|\cdot\|_{1}, \ldots,\|\cdot\|_{k}$, then the function $\|\varphi\|=\left\|\left(\|\varphi\|_{1}, \ldots,\|\varphi\|_{k}\right)\right\|_{*}$ is an RPI-norm, too. E.g., the function $\sqrt{\max |\varphi|^{2}+V_{\varphi}^{2}}$ is an RPI-norm. The reason for the hypothesis on $\|\cdot\|_{*}$ can be seen by taking the norm $\left\|\left(x_{1}, x_{2}\right)\right\|_{*}=\sqrt{x_{1}^{2}+\left(x_{1}-x_{2}\right)^{2}}$ on $\mathbb{R}^{2}$, and setting $\|\varphi\|_{1}=\max |\varphi|,\|\varphi\|_{2}=V_{\varphi}$. Indeed, in this case, $\|\cdot\|_{*}$ does not verify our hypothesis, and $F(\varphi)=\sqrt{\max |\varphi|^{2}+\left(\max |\varphi|-V_{\varphi}\right)^{2}}$ is not a norm on $A S^{1}(\mathbb{R})$, since the triangle inequality does not hold (e.g., $F\left(\varphi_{1}+\varphi_{2}\right)>F\left(\varphi_{1}\right)+F\left(\varphi_{2}\right)$ if $\varphi_{1}(t)=\Lambda(t)$ and $\varphi_{2}=\Lambda(t-4)$, where $\Lambda$ is the function defined in the next Definition 2.5).

Finally, another way to obtain an RPI-norm comes from the K-method in the Theory of Interpolation Spaces (cf. [3, 8]). Let us consider two RPI-norms $\|\cdot\|_{1}$, $\|\cdot\|_{2}$ and the function $K_{p}(t, \varphi)=\inf \left(\left\|\varphi_{1}\right\|_{1}^{p}+t^{p} \cdot\left\|\varphi_{2}\right\|_{2}^{p}\right)^{\frac{1}{p}}$, where $t, p>0$ and the infimum is computed for all possible decompositions $\varphi=\varphi_{1}+\varphi_{2}$ in $A S^{1}(\mathbb{R})$. Then $K_{p}(t, \cdot)$ is an RPI-norm.

Remark 2.4. Let $\|\cdot\|$ be an RPI-norm on $A S^{1}(\mathbb{R})$. If $\varphi$ has compact support, then also the composition $\varphi \circ h$ of $\varphi$ with an orientation-reversing $C^{1}$-diffeomorphism $h$ belongs to $A S^{1}(\mathbb{R})$. Hence it makes sense to ask if $\|\varphi\|$ equals $\|\varphi \circ h\|$ or not. In other words, the question is whether RPI-norms, that are invariant under orientation-preserving reparametrizations by definition, are invariant also under orientation-reversing reparametrizations, when restricted to the space $C_{c}^{1}(\mathbb{R})$. In general the answer is negative. As a counterexample, consider the RPI-norm $\|\varphi\|=\max _{t_{1} \leq t_{2}}\left|2 \varphi\left(t_{1}\right)-\varphi\left(t_{2}\right)\right|$.

In order to proceed, we need to introduce two useful almost sigmoidal functions, represented in Figure 3 , 

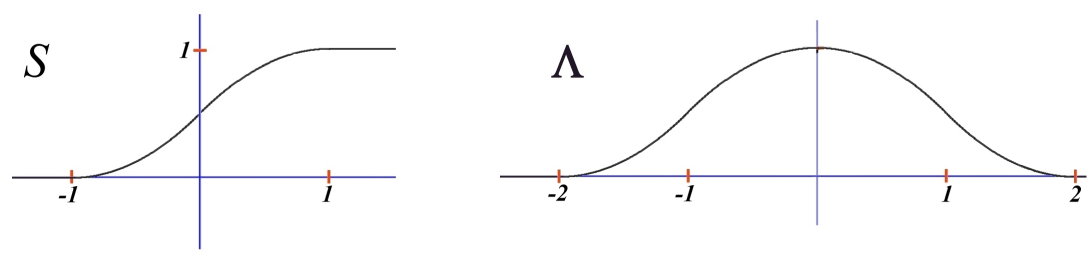

Figure 3. The graphs of the functions $S$ and $\Lambda$.

Definition 2.5. We shall denote by $S$ the almost sigmoidal $C^{1}$-function from $\mathbb{R}$ to $\mathbb{R}$ defined by setting

$$
S(t)= \begin{cases}0 & \text { if } t<-1 \\ \frac{(t+1)^{2}}{2} & \text { if }-1 \leq t \leq 0, \\ 1-\frac{(t-1)^{2}}{2} & \text { if } 0<t \leq 1 \\ 1 & \text { if } t>1\end{cases}
$$

We define $\Lambda: \mathbb{R} \rightarrow \mathbb{R}$ by setting $\Lambda(t)=S(t+1)-S(t-1)$.

In the following subsection we shall show that, in some sense, each RPI-norm is controlled by the norm of the function $S$.

2.2.1. The Bounding Lemma. The Bounding Lemma states that, after normalization, every RPI-norm of $\varphi$ is bounded from above by the total variation of $\varphi$, and from below by $\lim _{t \rightarrow+\infty}|\varphi(t)|$.

This result will be proved as a consequence of the fact that the increasing functions of $A S^{1}(\mathbb{R})$ can be approximated arbitrarily well by functions equivalent to multiples of the function $S$ (Prop. 2.6). From this, it follows that the RPI-norms of monotone functions are multiples of the norm of $S$ (Prop. 2.7).

Proposition 2.6. Assume that an RPI-norm $\|\cdot\|$ is given. For any increasing function $\varphi \in A S^{1}(\mathbb{R})$ and any $\varepsilon>0$, an increasing function $\varphi_{\varepsilon} \in A S^{1}(\mathbb{R})$ exists such that $\left\|\varphi_{\varepsilon}-\varphi\right\|=\varepsilon, \max \varphi_{\varepsilon}=\max \varphi+\frac{\varepsilon}{\|S\|}$ and $\varphi_{\varepsilon} \sim \max \varphi_{\varepsilon} \cdot S$.

Proof. Let $a$ and $b$ be two real numbers such that $a<b, \varphi$ equals 0 in the interval $(-\infty, a]$ and it is constant in the interval $[b,+\infty)$. Let us define $\widetilde{S}(t)=$ $S\left(2 \frac{t-a+\varepsilon}{b-a+2 \varepsilon}-1\right)$ and $\varphi_{\varepsilon}=\varphi+\frac{\varepsilon}{\|S\|} \cdot \widetilde{S}$. We point out that $\|\widetilde{S}\|=\|S\|$, since $\widetilde{S}$ is obtained by reparametrizing $S$. Hence $\left\|\varphi_{\varepsilon}-\varphi\right\|=\frac{\varepsilon}{\|S\|} \cdot\|\widetilde{S}\|=\varepsilon$. Moreover $\varphi_{\varepsilon}$ is clearly an increasing function belonging to $A S^{1}(\mathbb{R})$ and $\max \varphi_{\varepsilon}=\max \varphi+\frac{\varepsilon}{\|S\|}$.

Therefore, we only have to prove that a reparametrization $h \in D_{+}^{1}(\mathbb{R})$ exists, such that $\varphi_{\varepsilon}(t)=\max \varphi_{\varepsilon} \cdot S(h(t))$ for every $t \in \mathbb{R}$. In order to show this, we set

$$
c=\frac{2}{b-a+2 \varepsilon} \cdot \sqrt{\frac{\varepsilon}{\max \varphi_{\varepsilon} \cdot\|S\|}}
$$


and define

$$
h(t)= \begin{cases}c \cdot(t-a+\varepsilon)-1 & \text { if } t \leq a-\varepsilon, \\ f_{\varepsilon}\left(\varphi_{\varepsilon}(t)\right) & \text { if } a-\varepsilon<t<b+\varepsilon, \\ c \cdot(t-b-\varepsilon)+1 & \text { if } b+\varepsilon \leq t\end{cases}
$$

where $f_{\varepsilon}:\left[0, \max \varphi_{\varepsilon}\right] \rightarrow[-1,1]$ denotes the inverse function of the restriction of $\max \varphi_{\varepsilon} \cdot S$ to the interval $[-1,1]$. Note that $\max \varphi_{\varepsilon}=\max \varphi+\frac{\varepsilon}{\|S\|}$ is always positive. The definition of $f_{\varepsilon}$ implies that each $t \in(a-\varepsilon, b+\varepsilon)$ is taken by $h$ to the unique point $h(t)$ for which

$$
\varphi_{\varepsilon}(t)=\max \varphi_{\varepsilon} \cdot S(h(t)) .
$$

On the one hand, we observe that if $t \leq a$, then the equality $\varphi_{\varepsilon}(t)=\frac{\varepsilon}{\|S\|} \cdot \widetilde{S}(t)$ holds, and hence for $a-\varepsilon<t \leq a$ the equality (11) becomes

$$
\frac{\varepsilon}{\|S\|} \cdot \widetilde{S}(t)=\max \varphi_{\varepsilon} \cdot S(h(t))
$$

If $t$ is also close enough to $a-\varepsilon$ we have from (2) that $-1 \leq h(t) \leq 0$ and in this case $\widetilde{S}(t)=2\left(\frac{t-a+\varepsilon}{b-a+2 \varepsilon}\right)^{2}$ and $S(h(t))=\frac{(h(t)+1)^{2}}{2}$, because of the definitions of $\widetilde{S}$ and $S$. Then, by a direct computation, we obtain from (2) that if $a-\varepsilon<t \leq a$ and $t$ is close enough to $a-\varepsilon$, then the equality $h(t)=c \cdot(t-a+\varepsilon)-1$ holds.

On the other hand, if $t \geq b$, the equality $\varphi_{\varepsilon}(t)=\max \varphi+\frac{\varepsilon}{\|S\|} \cdot \widetilde{S}(t)$ holds, and hence for $b \leq t<b+\varepsilon$ the equality (11) becomes

$$
\max \varphi+\frac{\varepsilon}{\|S\|} \cdot \widetilde{S}(t)=\max \varphi_{\varepsilon} \cdot S(h(t)) .
$$

If $t$ is also close enough to $b+\varepsilon$, we have from (3) that $0 \leq h(t) \leq 1$ and, in this case, $\widetilde{S}(t)=1-2\left(\frac{t-b-\varepsilon}{b-a+2 \varepsilon}\right)^{2}$ and $S(h(t))=1-\frac{(h(t)-1)^{2}}{2}$, once more because of the definitions of $\widetilde{S}$ and $S$. Then, by a direct computation (recalling that $\max \varphi_{\varepsilon}=$ $\max \varphi+\frac{\varepsilon}{\|S\|}$ ) we obtain from (3) that if $b \leq t<b+\varepsilon$ and $t$ is close enough to $b+\varepsilon$, then the equality $h(t)=c \cdot(t-b-\varepsilon)+1$ holds.

It follows that $h$ is differentiable at both the points $a-\varepsilon$ and $b+\varepsilon$, and that at both of them the derivative of $h$ takes the positive value $c$.

Furthermore, we observe that the restriction of $h$ to the open interval $(a-\varepsilon, b+\varepsilon)$ has a positive derivative, since both the derivative of $\varphi_{\varepsilon}$ is positive in this interval (due to the addend $\frac{\varepsilon}{\|S\|} \cdot \widetilde{S}$ ) and the derivative of $f_{\varepsilon}$ is positive in the open interval $\left(0, \max \varphi_{\varepsilon}\right)$. Also, $h$ obviously has derivative equal to the positive value $c$ outside the interval $[a-\varepsilon, b+\varepsilon]$, because of its definition, and at points $a$ and $b$, since it is $C^{1}$. In conclusion, we have shown that $h$ is an orientation-preserving $C^{1}$-diffeomorphism.

As a final step, it is easy to verify that $\varphi_{\varepsilon}(t)=\max \varphi_{\varepsilon} \cdot S(h(t))$ for every $t \in \mathbb{R}$. Indeed, we already know that $\varphi_{\varepsilon}(t)=\max \varphi_{\varepsilon} \cdot S(h(t))$ for $a-\varepsilon<t<b+\varepsilon$. For $t \leq a-\varepsilon$ we have $h(t) \leq-1$, and hence $\varphi_{\varepsilon}(t)=0=\max \varphi_{\varepsilon} \cdot S(h(t))$, while for $t \geq b+\varepsilon$ we have $h(t) \geq 1$, and hence $\varphi_{\varepsilon}(t)=\max \varphi_{\varepsilon}=\max \varphi_{\varepsilon} \cdot S(h(t))$.

Therefore $\varphi_{\varepsilon}$ is equivalent to the function $\max \varphi_{\varepsilon} \cdot S$ and our statement is proved.

Now we can prove the following simple but crucial result, underlining the importance of the function $S$. 
Proposition 2.7. Assume that an RPI-norm $\|\cdot\|$ is given. For any monotone function $\psi \in A S^{1}(\mathbb{R})$ we have that $\|\psi\|=\max |\psi| \cdot\|S\|$.

Proof. Set $\varphi=|\psi|$. By applying the previous Proposition 2.6 and the triangle inequality, we obtain that $\left|\max \varphi_{\varepsilon} \cdot\|S\|-\|\varphi\|\right|=\left|\left\|\varphi_{\varepsilon}\right\|-\|\varphi\|\right| \leq\left\|\varphi_{\varepsilon}-\varphi\right\|=\varepsilon$. By passing to the limit for $\varepsilon$ tending to 0 , we get the equality $\max \varphi \cdot\|S\|-\|\varphi\|=0$ and our statement is proved.

Remark 2.8. We have to justify our line of proof of Proposition 2.7 since the passage through Proposition 2.6 could appear a little cumbersome. The point is that the function $\frac{\varphi}{\max \varphi \cdot S}$ may not tend towards a positive finite constant for $t \rightarrow a^{+}$ or for $t \rightarrow b^{-}$. Furthermore, it may happen that the derivative of $\varphi$ vanishes in the open interval $(a, b)$. In these cases we cannot change directly $\varphi$ into $\max \varphi \cdot S$ by a reparametrization, i.e. by composing $\varphi$ with an (orientation-preserving) $C^{1}$ diffeomorphism. Hence we have to change $\varphi$ into an approximation $\varphi_{\varepsilon}$ that does not present the previous problems.

Now we are ready to prove the Bounding Lemma. It gives a lower bound and an upper bound for each RPI-norm, involving the norm of $S$.

Lemma 2.9 (Bounding Lemma). Let $\|\cdot\|: A S^{1}(\mathbb{R}) \rightarrow \mathbb{R}$ be a reparametrization invariant norm. Then, for every $\varphi \in A S^{1}(\mathbb{R})$, the following inequalities hold:

$$
\lim _{t \rightarrow+\infty}|\varphi(t)| \cdot\|S\| \leq\|\varphi\| \leq V_{\varphi} \cdot\|S\| .
$$

Proof. We can write $\varphi=V^{+}\langle\varphi\rangle-V^{-}\langle\varphi\rangle$, with $V^{+}\langle\varphi\rangle, V^{-}\langle\varphi\rangle \in A S^{1}(\mathbb{R})$. Hence

$$
\left|\left\|V^{+}\langle\varphi\rangle\right\|-\left\|V^{-}\langle\varphi\rangle\right\|\right| \leq\|\varphi\| \leq\left\|V^{+}\langle\varphi\rangle\right\|+\left\|V^{-}\langle\varphi\rangle\right\| .
$$

Since $V^{+}\langle\varphi\rangle, V^{-}\langle\varphi\rangle$ are increasing, Proposition 2.7 implies that $\left\|V^{+}\langle\varphi\rangle\right\|=$ $V_{\varphi}^{+} \cdot\|S\|$ and $\left\|V^{-}\langle\varphi\rangle\right\|=V_{\varphi}^{-} \cdot\|S\|$, and hence our statement is proved by recalling that $V_{\varphi}^{+}-V_{\varphi}^{-}=\lim _{t \rightarrow+\infty} \varphi(t)$.

Remark 2.10. The inequalities in the Bounding Lemma are sharp, as we can easily see by setting $\varphi=S$.

Corollary 2.11. Let $\|\cdot\|: A S^{1}(\mathbb{R}) \rightarrow \mathbb{R}$ be a reparametrization invariant norm. Then $\|\Lambda\| \leq 2 \cdot\|S\|$.

Proof. Set $\varphi=\Lambda$ in the previous Lemma 2.9

Remark 2.12. We observe that the inequality proved in Corollary 2.11 is sharp, since $\|\Lambda\|$ can equal $2 \cdot\|S\|$. For instance, this happens when we consider the total variation. Moreover, it is interesting to note that no positive constant $c$ exists such that the inequality $c \cdot\|S\| \leq\|\Lambda\|$ holds for every RPI-norm $\|\cdot\|$. To see this, it is sufficient to consider the RPI-norm $\|\varphi\|_{k}=\max |\varphi|+k \cdot \lim _{t \rightarrow+\infty}|\varphi(t)|$, for $k \geq 0$. Since $c \cdot\|S\|_{k}=c \cdot(1+k)$ and $\|\Lambda\|_{k}=1$, if $k$ is large enough, the inequality $c \cdot\|S\|_{k} \leq\|\Lambda\|_{k}$ does not hold. Also in this sense, the lower bound in the Bounding Lemma cannot be improved. Incidentally, we observe that the function $\lim _{t \rightarrow+\infty}|\varphi(t)|$ defines a seminorm on $A S^{1}(\mathbb{R})$ that is reparametrization invariant. 
2.2.2. Derivatives and RPI-norms. The main consequence of the Bounding Lemma is that the closeness of two almost sigmoidal functions with respect to the total variation norm implies their closeness with respect to any other RPI-norm. From this we obtain the next proposition, showing that if the derivatives of two functions $\varphi, \psi \in A S^{1}(\mathbb{R})$ are close to each other everywhere, then $\varphi$ and $\psi$ are close to each other with respect to any other reparametrization invariant norm.

Proposition 2.13. Let $\|\cdot\|$ be a reparametrization invariant norm on $A S^{1}(\mathbb{R})$. Assume that $\varphi, \psi \in A S^{1}(\mathbb{R})$ and that the compact support of their derivative is contained in the interval $[a, b]$ with $a \neq b$. If $\max \left|\frac{d \varphi}{d t}-\frac{d \psi}{d t}\right| \leq \frac{\varepsilon}{(b-a) \cdot\|S\|}$, then $\|\varphi-\psi\| \leq \varepsilon$.

Proof. If $\max \left|\frac{d \varphi}{d t}-\frac{d \psi}{d t}\right| \leq \frac{\varepsilon}{(b-a) \cdot\|S\|}$, we have that

$$
V_{\varphi-\psi}=\int_{-\infty}^{+\infty}\left|\frac{d(\varphi-\psi)}{d t}(t)\right| d t \leq \frac{\varepsilon}{(b-a) \cdot\|S\|} \cdot(b-a)=\frac{\varepsilon}{\|S\|} .
$$

By applying the right inequality in the Bounding Lemma 2.9 we obtain

$$
\|\varphi-\psi\| \leq V_{\varphi-\psi} \cdot\|S\| \leq \frac{\varepsilon}{\|S\|} \cdot\|S\|=\varepsilon .
$$

2.2.3. A stronger Bounding Lemma for functions in $C_{c}^{1}(\mathbb{R})$. The inequalities in the Bounding Lemma can be improved if $\varphi$ belongs to $C_{c}^{1}(\mathbb{R}) \subseteq A S^{1}(\mathbb{R})$. In this case $\|\varphi\|$ stays somewhere between the norms $\max |\varphi|$ and $\frac{1}{2} V_{\varphi}$, up to the multiplicative constant $\|\Lambda\|$. This section aims to prove these stronger bounds.

In order to prove these results we need the following technical lemma, where $V_{\chi}$ and $V_{\bar{\chi}-\chi}$ denote the total variation of $\chi$ and $\bar{\chi}-\chi$, respectively, on $[\alpha, \beta]$.

Lemma 2.14. Let $\chi:[\alpha, \beta] \rightarrow \mathbb{R}$ be a monotone function of class $C^{1}$ with $\chi(\alpha) \neq$ $\chi(\beta)$ and vanishing derivatives at $\alpha$ and $\beta$. Then, for any $\varepsilon>0, a C^{1}$-function $\bar{\chi}:[\alpha, \beta] \rightarrow \mathbb{R}$ and an orientation-preserving $C^{1}$-diffeomorphism $\bar{h}:[\alpha, \beta] \rightarrow[\alpha, \beta]$ exist such that

(1) if $\chi$ is increasing, then $\frac{d \bar{\chi}}{d t}>0$ in the open interval $(\alpha, \beta)$; if $\chi$ is decreasing, then $\frac{d \bar{\chi}}{d t}<0$ in $(\alpha, \beta)$;

(2) $V_{\bar{\chi}-\chi} \leq \varepsilon$;

(3) $\bar{\chi}(\bar{h}(t))=\chi(\alpha)+(\chi(\beta)-\chi(\alpha)) \cdot S\left(2 \cdot \frac{t-\alpha}{\beta-\alpha}-1\right)$ for every $t \in[\alpha, \beta]$ and there exists $\eta>0$ such that $\bar{h}$ is the identity on the intervals $[\alpha, \alpha+\eta]$ and $[\beta-\eta, \beta]$.

Proof. Let us define $\widehat{S}(t)=S\left(2 \cdot \frac{t-\alpha}{\beta-\alpha}-1\right)$. Let us choose two small values $\eta_{2}>$ $\eta_{1}>0$ and an $\hat{\varepsilon}>0$, and set

$$
\bar{\chi}(t)= \begin{cases}\chi(\alpha)+2 \cdot(t-\alpha)^{2} \cdot \frac{\chi(\beta)-\chi(\alpha)}{(\beta-\alpha)^{2}} & \text { if } t \in\left[\alpha, \alpha+\eta_{1}\right], \\ (\chi+\operatorname{sign}(\chi(\beta)-\chi(\alpha)) \cdot \hat{\varepsilon} \cdot \widehat{S}) \cdot \frac{V_{\chi}}{V_{\chi}+\hat{\varepsilon}} & \text { if } t \in\left[\alpha+\eta_{2}, \beta-\eta_{2}\right], \\ \chi(\beta)-2 \cdot(\beta-t)^{2} \cdot \frac{\chi(\beta)-\chi(\alpha)}{(\beta-\alpha)^{2}} & \text { if } t \in\left[\beta-\eta_{1}, \beta\right] .\end{cases}
$$

Let us notice that if $\chi$ is increasing, then $\bar{\chi}$ is also increasing in the intervals where it is defined, and, vice versa, if $\chi$ is decreasing, then $\bar{\chi}$ is also decreasing. Moreover, in the intervals where it is defined, the derivative of $\bar{\chi}$ does not vanish, except at $\alpha$ and $\beta$ (where $\chi$ and $\bar{\chi}$ coincide). 


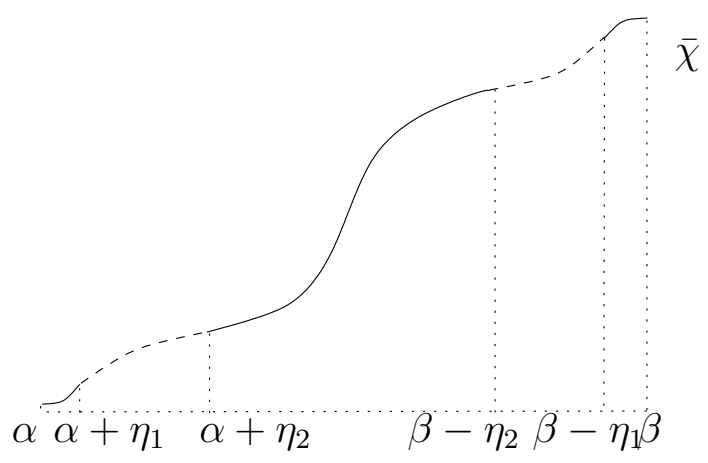

Figure 4. The function $\bar{\chi}$ used in Lemma 2.14 (case $\chi(\alpha)<$ $\chi(\beta))$ : it is quadratic near $\alpha$ and $\beta$, and without critical points in $(\alpha, \beta)$.

Now, if $\eta_{1}$ is small enough in comparison to $\eta_{2}$, we have that $\bar{\chi}\left(\alpha+\eta_{2}\right)-$ $\bar{\chi}\left(\alpha+\eta_{1}\right)$ is positive or negative according to whether $\chi$, and hence $\bar{\chi}$, is increasing or decreasing. Analogously, $\bar{\chi}\left(\beta-\eta_{1}\right)-\bar{\chi}\left(\beta-\eta_{2}\right)$ is positive or negative according to whether $\chi$, and hence $\bar{\chi}$, is increasing or decreasing. Therefore, we can extend the definition of $\bar{\chi}$ to the open intervals $\left(\alpha+\eta_{1}, \alpha+\eta_{2}\right),\left(\beta-\eta_{2}, \beta-\eta_{1}\right)$ in such a way that $\bar{\chi}$ is a $C^{1}$ - function with non-vanishing derivative in the open interval $(\alpha, \beta)$. Moreover, either $\bar{\chi}$ and $\chi$ are both increasing or they are both decreasing. So, $\bar{\chi}$ satisfies property (1).

Furthermore, if $\eta_{1}$ and $\eta_{2}$ have been chosen small enough, $\bar{\chi}$ also satisfies property (2). Indeed,

$$
\begin{aligned}
& V_{\bar{\chi}-\chi}=\int_{\alpha}^{\alpha+\eta_{2}}\left|\frac{d(\bar{\chi}-\chi)}{d t}\right| d t+\int_{\alpha+\eta_{2}}^{\beta-\eta_{2}}\left|\frac{d(\bar{\chi}-\chi)}{d t}\right| d t+\int_{\beta-\eta_{2}}^{\beta}\left|\frac{d(\bar{\chi}-\chi)}{d t}\right| d t \\
& =\int_{\alpha}^{\alpha+\eta_{2}}\left|\frac{d(\bar{\chi}-\chi)}{d t}\right| d t+\frac{\hat{\varepsilon}}{V_{\chi}+\hat{\varepsilon}} \\
& \quad \cdot \int_{\alpha+\eta_{2}}^{\beta-\eta_{2}}\left|\operatorname{sign}(\chi(\beta)-\chi(\alpha)) \cdot V_{\chi} \cdot \frac{d \widehat{S}}{d t}-\frac{d \chi}{d t}\right| d t+\int_{\beta-\eta_{2}}^{\beta}\left|\frac{d(\bar{\chi}-\chi)}{d t}\right| d t \\
& \leq \int_{\alpha}^{\alpha+\eta_{2}}\left|\frac{d \bar{\chi}}{d t}\right| d t \\
& \quad+\int_{\alpha}^{\alpha+\eta_{2}}\left|\frac{d \chi}{d t}\right| d t+\frac{\hat{\varepsilon}}{V_{\chi}+\hat{\varepsilon}} \cdot\left(\int_{\alpha+\eta_{2}}^{\beta-\eta_{2}}\left|V_{\chi} \cdot \frac{d \widehat{S}}{d t}\right| d t+\int_{\alpha+\eta_{2}}^{\beta-\eta_{2}}\left|\frac{d \chi}{d t}\right| d t\right) \\
& \quad+\int_{\beta-\eta_{2}}^{\beta}\left|\frac{d \bar{\chi}}{d t}\right| d t+\int_{\beta-\eta_{2}}^{\beta}\left|\frac{d \chi}{d t}\right| d t \\
& \leq\left|\bar{\chi}\left(\alpha+\eta_{2}\right)-\bar{\chi}(\alpha)\right|+\left|\chi\left(\alpha+\eta_{2}\right)-\chi(\alpha)\right|+\frac{\hat{\varepsilon}}{V_{\chi}+\hat{\varepsilon}} \cdot 2 \cdot V_{\chi} \\
& \quad+\left|\bar{\chi}(\beta)-\bar{\chi}\left(\beta-\eta_{2}\right)\right|+\left|\chi(\beta)-\chi\left(\beta-\eta_{2}\right)\right| .
\end{aligned}
$$


Therefore, taking $\eta_{2}$ (and hence $\eta_{1}$ ) small enough, by continuity we obtain that

$$
V_{\bar{\chi}-\chi} \leq 2 \cdot \frac{\hat{\varepsilon}}{V_{\chi}+\hat{\varepsilon}} \cdot V_{\chi}+4 \hat{\varepsilon} .
$$

It follows that if we choose $\hat{\varepsilon}, \eta_{1}$ and $\eta_{2}$ small enough, then the inequality $V_{\bar{\chi}-\chi} \leq \varepsilon$ holds.

As for statement (3), because of (1), in the open interval $(\alpha, \beta), \bar{\chi}$ admits the $C^{1}$ inverse function $\bar{\chi}^{-1}$. We define $\hat{h}:(\alpha, \beta) \rightarrow(\alpha, \beta)$ by setting

$$
\hat{h}=\bar{\chi}^{-1} \circ(\chi(\alpha)+(\chi(\beta)-\chi(\alpha)) \cdot \hat{S}) .
$$

Now, $\hat{h}$ is an orientation-preserving $C^{1}$-diffeomorphism because $\bar{\chi}$ and $(\chi(\alpha)+(\chi(\beta)-\chi(\alpha)) \cdot \hat{S})$ are both increasing or both decreasing $C^{1}$-functions with non-vanishing derivatives.

Now, by taking $\eta<\eta_{1}$, we obtain that $\hat{h}$ is the identity on $(\alpha, \alpha+\eta] \cup[\beta-\eta, \beta)$. This fact can be verified by direct computation. Here the key point is that in the intervals $\left[\alpha, \alpha+\eta_{1}\right]$ and $\left[\beta-\eta_{1}, \beta\right]$ the function $\bar{\chi}$ has been defined to be quadratic like $\hat{S}$. Therefore, if we extend $\hat{h}$ to the closed interval $[\alpha, \beta]$ by taking $\bar{h}:[\alpha, \beta] \rightarrow[\alpha, \beta]$ with $\bar{h}(\alpha)=\alpha, \bar{h}(\beta)=\beta$, and $\bar{h}(t)=\hat{h}(t)$ for $t \in(\alpha, \beta)$, then $\bar{h}$ is an orientation-preserving $C^{1}$-diffeomorphism defined in $[\alpha, \beta]$, satisfying condition (3).

Now we can prove the following result for $\Lambda$, analogous to Proposition 2.7proved for $S$.

Proposition 2.15. Assume that an RPI-norm $\|\cdot\|$ is given. Assume also that $\varphi \in C_{c}^{1}(\mathbb{R})$, and that a value $\bar{t} \in \mathbb{R}$ exists such that $\varphi$ is monotone both in $(-\infty, \bar{t}]$ and in $[\bar{t},+\infty)$. Then $\|\varphi\|=\max |\varphi| \cdot\|\Lambda\|$.

Proof. Let us assume that $\varphi \neq \mathbf{0}$; otherwise the claim is trivial. Consider the smallest interval $[a, b]$ containing the compact support of $\frac{d \varphi}{d t}$. Possibly by taking $-\varphi$ instead of $\varphi$, we can also assume that $\varphi$ is increasing in $[a, \bar{t}]$ and decreasing in $[\bar{t}, b]$, so that $\varphi \geq 0$. Furthermore, up to a reparametrization, we can assume that $a=-2, \bar{t}=0$ and $b=2$.

Let $\chi_{1}$ denote the restriction of $\varphi$ to the interval $[-2,0]$ and $\chi_{2}$ denote the restriction of $\varphi$ to the interval $[0,2]$. Let us apply Lemma 2.14 for some $\varepsilon>0$ in order to obtain two functions $\bar{\chi}_{1}$ and $\bar{\chi}_{2}$ and the diffeomorphisms $\bar{h}_{1}$ and $\bar{h}_{2}$ such that $V_{\bar{\chi}_{1}-\chi_{1}} \leq \frac{\varepsilon}{2}, V_{\bar{\chi}_{2}-\chi_{2}} \leq \frac{\varepsilon}{2}, \bar{\chi}_{1}\left(\bar{h}_{1}(t)\right)=\varphi(0) \cdot S\left(2 \cdot \frac{t+2}{2}-1\right)=\max |\varphi| \cdot S(t+1)$ and $\bar{\chi}_{2}\left(\bar{h}_{2}(t)\right)=\varphi(0)-\varphi(0) \cdot S\left(2 \cdot \frac{t}{2}-1\right)=\max |\varphi|-\max |\varphi| \cdot S(t-1)$. Recall also that $\bar{h}_{1}$ is the identity in a neighbourhood of -2 and 0 , and $\bar{h}_{2}$ is the identity in a neighbourhood of 0 and 2 .

Consider the function $\varphi_{\varepsilon}: \mathbb{R} \rightarrow \mathbb{R}$ in $A S^{1}(\mathbb{R})$ defined by

$$
\varphi_{\varepsilon}(t)= \begin{cases}0 & \text { if } t \leq-2, \\ \bar{\chi}_{1}(t) & \text { if }-2<t \leq 0, \\ \bar{\chi}_{2}(t) & \text { if } 0<t \leq 2, \\ 0 & \text { if } t>2\end{cases}
$$

We have that $\varphi_{\varepsilon}$ is a function in $C_{c}^{1}(\mathbb{R})$, with $V_{\varphi_{\varepsilon}-\varphi} \leq \varepsilon$. So, by applying the Bounding Lemma 2.9, we deduce that $\left\|\varphi_{\varepsilon}-\varphi\right\| \leq \varepsilon \cdot\|S\|$.

Let us consider the orientation-preserving $C^{1}$-diffeomorphism $h: \mathbb{R} \rightarrow \mathbb{R}$, defined by setting $h(t)=\bar{h}_{1}(t)$ for $t \in[-2,0], h(t)=\bar{h}_{2}(t)$ for $t \in[0,2], h(t)=t$ otherwise. 
It holds that $\varphi_{\varepsilon}(h(t))=\max |\varphi| \cdot S(t+1)-\max |\varphi| \cdot S(t-1)=\max |\varphi| \cdot \Lambda$. Therefore, $|\max \varphi \cdot\|\Lambda\|-\|\varphi\||=\left|\left\|\varphi_{\varepsilon}\right\|-\|\varphi\|\right| \leq\left\|\varphi_{\varepsilon}-\varphi\right\| \leq \varepsilon \cdot\|S\|$. By passing to the limit for $\varepsilon$ tending to 0 , we get the equality $\max \varphi \cdot\|\Lambda\|-\|\varphi\|=0$ and our statement is proved.

The following result will be useful in the proof of the Reconstruction Theorem 5.8. We omit its proof, being quite similar to the ones used for Lemma 2.14 and Proposition 2.15.

Proposition 2.16. Let $\varphi \in A S^{1}(\mathbb{R})$ admit n points $t_{0}<t_{1}<\ldots<t_{n-1}$ such that $\varphi$ is monotone in each of the intervals $\left(-\infty, t_{0}\right],\left[t_{0}, t_{1}\right], \ldots,\left[t_{n-2}, t_{n-1}\right],\left[t_{n-1},+\infty\right)$. Then, for any $\varepsilon>0$, an $A S^{1}$-function $\varphi_{\varepsilon}: \mathbb{R} \rightarrow \mathbb{R}$ exists such that

(1) $V_{\varphi_{\varepsilon}-\varphi} \leq \varepsilon$;

(2) $\varphi_{\varepsilon} \sim \varphi\left(t_{0}\right) \cdot S(t)+\sum_{i=1}^{n-1}\left(\varphi\left(t_{i}\right)-\varphi\left(t_{i-1}\right)\right) \cdot S(t-2 i)$.

In particular, $\|\varphi\|=\left\|\varphi\left(t_{0}\right) \cdot S(t)+\sum_{i=1}^{n-1}\left(\varphi\left(t_{i}\right)-\varphi\left(t_{i-1}\right)\right) \cdot S(t-2 i)\right\|$ for any RPI-norm $\|\cdot\|$.

Now we are ready to prove the stronger version of the Bounding Lemma for functions with compact support. It gives a lower bound and an upper bound for each RPI-norm, involving the norm of $\Lambda$.

Lemma 2.17 (Bounding Lemma for $C_{c}^{1}(\mathbb{R})$ ). Let $\|\cdot\|: A S^{1}(\mathbb{R}) \rightarrow \mathbb{R}$ be a reparametrization invariant norm. Then, for every $\varphi \in C_{c}^{1}(\mathbb{R})$, the following inequalities hold:

$$
\max |\varphi| \cdot\|\Lambda\| \leq\|\varphi\| \leq \frac{1}{2} V_{\varphi} \cdot\|\Lambda\| .
$$

Proof. First of all we prove the left inequality. We take a point $t_{\max }$ where $|\varphi|$ takes its maximum value and consider the function

$$
\psi(\tau)= \begin{cases}\int_{-\infty}^{\tau}\left|\frac{d \varphi}{d t}(t)\right|+\left|\frac{d \varphi}{d t}\left(2 t_{\max }-t\right)\right| d t & \text { if } \tau \leq t_{\max } \\ \int_{\tau}^{+\infty}\left|\frac{d \varphi}{d t}(t)\right|+\left|\frac{d \varphi}{d t}\left(2 t_{\max }-t\right)\right| d t & \text { if } \tau>t_{\max }\end{cases}
$$

and set $\hat{\varphi}=\varphi+\psi$.

We can easily verify that $\psi$ is continuous also at $t_{\max }$, because of the two addends appearing in its definition. Then we observe that both $\hat{\varphi}$ and $\psi$ belong to $C_{c}^{1}(\mathbb{R})$. In particular, the regularity of $\psi$ follows from the fact that $t_{\max }$ is a critical point for $\varphi$. Moreover, by computing their derivative, we see that $\hat{\varphi}$ and $\psi$ are increasing in $\left(-\infty, t_{\max }\right]$ and decreasing in $\left[t_{\max },+\infty\right)$. Furthermore, $\max \hat{\varphi}=\hat{\varphi}\left(t_{\max }\right)=$ $\operatorname{sign}\left(\varphi\left(t_{\max }\right)\right) \cdot \max |\varphi|+\max \psi$.

Since $\varphi=\hat{\varphi}-\psi$, by applying Proposition 2.15 with $\bar{t}=t_{\max }$ we get

As for the proof of the other inequality, we begin by considering an interval $[a, b]$ with $a \neq b$, such that the compact support of $\frac{d \varphi}{d t}$ is contained in $[a, b]$. Let us define the function

$$
F(\tau)=\int_{-\infty}^{\tau} \max \left\{\frac{d \varphi}{d t}(t), 0\right\} d t-\int_{\tau}^{+\infty} \max \left\{-\frac{d \varphi}{d t}(t), 0\right\} d t .
$$


Since $F(a)=-V_{\varphi}^{-} \leq 0, F(b)=V_{\varphi}^{+} \geq 0$ and $F$ is continuous, a value $\bar{t} \in[a, b]$ exists such that $F(\bar{t})=0$, i.e.,

$$
\int_{-\infty}^{\bar{t}} \max \left\{\frac{d \varphi}{d t}(t), 0\right\} d t=\int_{\bar{t}}^{+\infty} \max \left\{-\frac{d \varphi}{d t}(t), 0\right\} d t .
$$

Let us now assume that $\frac{d \varphi}{d t}(\bar{t})=0$. In this case we set

$$
\varphi_{1}(\tau)= \begin{cases}\int_{-\infty}^{\tau} \max \left\{\frac{d \varphi}{d t}(t), 0\right\} d t & \text { if } \tau \leq \bar{t} \\ \int_{\tau}^{+\infty} \max \left\{-\frac{d \varphi}{d t}(t), 0\right\} d t & \text { if } \tau>\bar{t}\end{cases}
$$

and

$$
\varphi_{2}(\tau)= \begin{cases}\int_{-\infty}^{\tau} \max \left\{-\frac{d \varphi}{d t}(t), 0\right\} d t & \text { if } \tau \leq \bar{t} \\ \int_{\tau}^{+\infty} \max \left\{\frac{d \varphi}{d t}(t), 0\right\} d t & \text { if } \tau>\bar{t}\end{cases}
$$

Since $\int_{-\infty}^{+\infty} \frac{d \varphi}{d t}(t) d t=0$, it is immediate to verify that $\varphi_{1}(\tau)-\varphi_{2}(\tau)=$ $\int_{-\infty}^{\tau} \frac{d \varphi}{d t}(t) d t=\varphi(\tau)$ for every $\tau \in \mathbb{R}$. Because of the choice of $\bar{t}, \varphi_{1}$ and $\varphi_{2}$ are continuous also at $\bar{t}$. Moreover, we observe that both $\varphi_{1}$ and $\varphi_{2}$ are $C_{c}^{1}(\mathbb{R})$ functions (here we are using the hypothesis $\frac{d \varphi}{d t}(\bar{t})=0$ ). Furthermore, they are increasing in $(-\infty, \bar{t}]$ and decreasing in $[\bar{t},+\infty)$.

By applying Proposition 2.15 we get

$$
\begin{aligned}
\|\varphi\| & =\left\|\varphi_{1}-\varphi_{2}\right\| \leq\left\|\varphi_{1}\right\|+\left\|\varphi_{2}\right\| \\
& =\max \varphi_{1} \cdot\|\Lambda\|+\max \varphi_{2} \cdot\|\Lambda\| \\
& =\left(\max \varphi_{1}+\max \varphi_{2}\right) \cdot\|\Lambda\| \\
& =\left(\int_{-\infty}^{\bar{t}} \max \left\{\frac{d \varphi}{d t}(t), 0\right\} d t+\int_{\bar{t}}^{+\infty} \max \left\{\frac{d \varphi}{d t}(t), 0\right\} d t\right) \cdot\|\Lambda\| \\
& =\left(\int_{-\infty}^{+\infty} \max \left\{\frac{d \varphi}{d t}(t), 0\right\} d t\right) \cdot\|\Lambda\| \\
& =V_{\varphi}^{+} \cdot\|\Lambda\|=\frac{1}{2} V_{\varphi} \cdot\|\Lambda\| .
\end{aligned}
$$

Therefore the inequality $\|\varphi\| \leq \frac{1}{2} V_{\varphi} \cdot\|\Lambda\|$ is proved, in the case when $\frac{d \varphi}{d t}(\bar{t})=0$. Otherwise, if $\frac{d \varphi}{d t}(\bar{t}) \neq 0$, we observe that for every $\varepsilon>0, \varphi$ can be locally modified near $\bar{t}$ into a function $\varphi_{\varepsilon} \in C_{c}^{1}(\mathbb{R})$ such that

- $V_{\varphi-\varphi_{\varepsilon}} \leq \varepsilon$

- $\frac{d \varphi_{\varepsilon}}{d t}(\bar{t})=0$,

- $\int_{-\infty}^{\bar{t}} \max \left\{\frac{d \varphi_{\varepsilon}}{d t}(t), 0\right\} d t=\int_{\bar{t}}^{+\infty} \max \left\{-\frac{d \varphi_{\varepsilon}}{d t}(t), 0\right\} d t$.

The change we are using is represented in Figure 5.

Because of what we have just proved in the case $\frac{d \varphi}{d t}(\bar{t})=0$, it follows that $\left\|\varphi_{\varepsilon}\right\| \leq \frac{1}{2} V_{\varphi_{\varepsilon}} \cdot\|\Lambda\|$ and hence $\left\|\varphi_{\varepsilon}\right\| \leq \frac{1}{2}\left(V_{\varphi}+\varepsilon\right) \cdot\|\Lambda\|\left(\right.$ since $\left.\left|V_{\varphi}-V_{\varphi_{\varepsilon}}\right| \leq V_{\varphi-\varphi_{\varepsilon}} \leq \varepsilon\right)$.

Now the Bounding Lemma 2.9 ensures that

$$
\left|\|\varphi\|-\left\|\varphi_{\varepsilon}\right\|\right| \leq\left\|\varphi-\varphi_{\varepsilon}\right\| \leq V_{\varphi-\varphi_{\varepsilon}} \cdot\|S\| \leq \varepsilon \cdot\|S\|
$$

and hence

$$
\|\varphi\|-\varepsilon \cdot\|S\| \leq \frac{1}{2}\left(V_{\varphi}+\varepsilon\right) \cdot\|\Lambda\|
$$




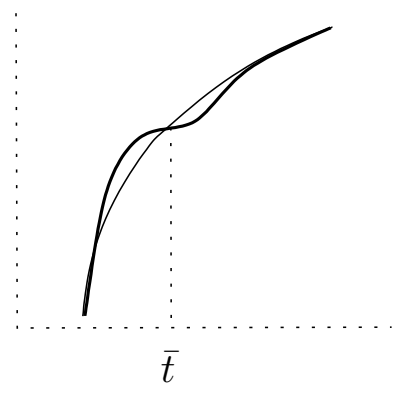

Figure 5. The change from $\varphi$ (thin) to $\varphi_{\varepsilon}$ (thick) in order to get $\frac{d \varphi_{\varepsilon}}{d t}(\bar{t})=0$ in the proof of the Bounding Lemma for $C_{c}^{1}(\mathbb{R})$.

Then the right inequality is proved for any $\varphi$, passing to the limit for $\varepsilon$ tending to 0 .

Remark 2.18. The double inequality that we have just proved shows that, if we confine ourselves to considering functions in $C_{c}^{1}(\mathbb{R})$, half the total variation and $\max |\varphi|$ are the two extreme cases of RPI-norms. All other RPI-norms are somewhere between them, after normalization with respect to $\Lambda$. We also observe that the two new inequalities are sharp, as we can immediately verify by setting $\|\varphi\|=\max |\varphi|$ and $\|\varphi\|=V_{\varphi}$.

Remark 2.19. While the lower bound in the Bounding Lemma 2.9 vanishes for all functions in $C_{c}^{1}(\mathbb{R})$, the lower bound in Lemma 2.17 never vanishes for non-zero functions in $C_{c}^{1}(\mathbb{R})$. This difference makes the study of functions with compact support easier than the study of general almost sigmoidal functions.

Remark 2.20. The Bounding Lemma for functions with compact support allows us to obtain new bounds for the RPI-norms on $A S^{1}(\mathbb{R})$. More precisely, if $\varphi \in A S^{1}(\mathbb{R})$ and $l=\lim _{t \rightarrow+\infty} \varphi(t)$, we have that for any RPI-norm $\|\cdot\|$ on $A S^{1}(\mathbb{R})$ it holds that

$$
\max |\varphi|-|l| \cdot\left(1+\frac{\|S\|}{\|\Lambda\|}\right) \leq \frac{\|\varphi\|}{\|\Lambda\|} \leq \frac{1}{2} V_{\varphi}+|l| \cdot\left(\frac{1}{2}+\frac{\|S\|}{\|\Lambda\|}\right) .
$$

This can be proved by estimating the norm of the function with compact support $\hat{\varphi}=\varphi-l \cdot S$.

2.3. Can a reparametrization invariant norm be induced by an inner product? We consider the question of whether a reparametrization invariant norm can be associated with some inner product. The next result shows that the answer to this question is negative.

Proposition 2.21. No inner product on $A S^{1}(\mathbb{R})$ can induce a reparametrization invariant norm.

Proof. Assume that an inner product $\langle\cdot, \cdot\rangle$ exists on $A S^{1}(\mathbb{R})$, inducing a reparametrization invariant norm. The associated norm $\|\cdot\|$ satisfies the parallelogram identity:

$$
\left\|\varphi_{1}+\varphi_{2}\right\|^{2}+\left\|\varphi_{1}-\varphi_{2}\right\|^{2}=2\left(\left\|\varphi_{1}\right\|^{2}+\left\|\varphi_{2}\right\|^{2}\right) .
$$

Let us take an almost sigmoidal function $\varphi$ with compact support, and set $\varphi_{1}=$ $V^{+}\langle\varphi\rangle$ and $\varphi_{2}=V^{-}\langle\varphi\rangle$. Then $\varphi_{1}+\varphi_{2}=V\langle\varphi\rangle, \varphi_{1}-\varphi_{2}=\varphi$. By applying (5) and 
Proposition 2.7 pertaining to the norm of monotone almost sigmoidal functions we get

$$
\begin{aligned}
\|\varphi\|^{2} & =2\left(\left\|V^{+}\langle\varphi\rangle\right\|^{2}+\left\|V^{-}\langle\varphi\rangle\right\|^{2}\right)-\|V\langle\varphi\rangle\|^{2} \\
& =2\left(V_{\varphi}^{+}\right)^{2} \cdot\|S\|^{2}+2\left(V_{\varphi}^{-}\right)^{2} \cdot\|S\|^{2}-\left(V_{\varphi}^{+}+V_{\varphi}^{-}\right)^{2} \cdot\|S\|^{2} \\
& =\left(\left(V_{\varphi}^{+}\right)^{2}+\left(V_{\varphi}^{-}\right)^{2}-2\left(V_{\varphi}^{+}\right)\left(V_{\varphi}^{-}\right)\right) \cdot\|S\|^{2} \\
& =\left(V_{\varphi}^{+}-V_{\varphi}^{-}\right)^{2} \cdot\|S\|^{2} .
\end{aligned}
$$

Since for every $\varphi$ with compact support we have that $V_{\varphi}^{+}-V_{\varphi}^{-}=0$, every function of this type should have a vanishing norm. This contradicts the definition of norm.

However, we remark that there exist degenerate symmetric bilinear maps $\Phi$ inducing reparametrization invariant semi-norms on $A S^{1}(\mathbb{R})$. An example is given by

$$
\Phi(\varphi, \psi)=\lim _{t \rightarrow+\infty} \varphi(t) \cdot \lim _{t \rightarrow+\infty} \psi(t)
$$

\section{STANDARD REPARAMETRIZATION INVARIANT NORMS}

In this section we introduce a class of reparametrization invariant norms on $A S^{1}(\mathbb{R})$. One well-known norm belonging to this class is the $L_{\infty}$-norm. For the sake of conciseness and clearness in our explanation, for every $\varphi \in A S^{1}(\mathbb{R})$ we shall often use the symbol $\varphi^{*}$ to denote the function $\varphi^{*}(t)=\varphi(-t)$. Obviously, in general, $\varphi^{*}$ is not an almost sigmoidal function, since it is obtained by composing $\varphi$ with an orientation-reversing diffeomorphism of $\mathbb{R}$.

\subsection{The integral definition.}

Lemma 3.1. Let $\varphi, \psi \in A S^{1}(\mathbb{R})$. The following statements hold:

i):

$$
\int_{-\infty}^{+\infty} \varphi^{*}(t) \cdot \frac{d \psi}{d t}(t) d t=-\int_{-\infty}^{+\infty} \frac{d \varphi^{*}}{d t}(t) \cdot \psi(t) d t
$$

ii):

$$
\left|\int_{-\infty}^{+\infty} \varphi^{*}(t) \cdot \frac{d \psi}{d t}(t) d t\right| \leq \max |\varphi| \cdot V_{\psi}
$$

Proof. i): Integrate by parts and observe that $\left.\varphi^{*}(t) \cdot \psi(t)\right|_{-\infty} ^{+\infty}=0$.

ii):

$$
\left|\int_{-\infty}^{+\infty} \varphi^{*}(t) \cdot \frac{d \psi}{d t}(t) d t\right| \leq \max |\varphi| \cdot \int_{-\infty}^{+\infty}\left|\frac{d \psi}{d t}(t)\right| d t=\max |\varphi| \cdot V_{\psi} .
$$

Theorem 3.2. For every $\varphi, \psi \in A S^{1}(\mathbb{R})$, let us set

$$
\|\varphi\|_{[\psi]}=\sup _{\hat{\psi} \in[\psi]}\left|\int_{-\infty}^{+\infty} \varphi(-t) \cdot \frac{d \hat{\psi}}{d t}(t) d t\right| .
$$



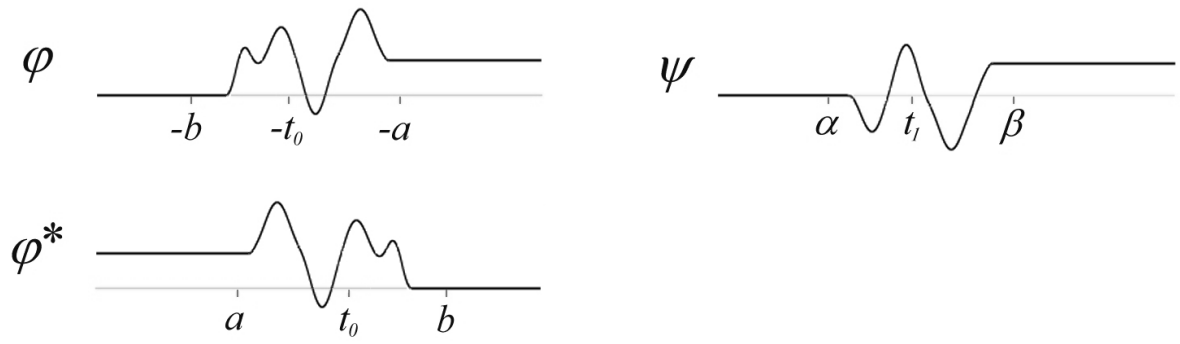

Figure 6. The functions $\varphi, \varphi^{*}$ and $\psi$ (proof of Theorem 3.2).

\section{It holds that}

(1) $\|\varphi\|_{[\psi]}=\|\varphi\|_{[-\psi]}$;

(2) $\|\varphi\|_{[\psi]}=\|\psi\|_{[\varphi]}$;

(3) $\|\varphi\|_{[\psi]} \leq \min \left\{\max |\varphi| \cdot V_{\psi}, \max |\psi| \cdot V_{\varphi}\right\}$.

Moreover, if $\psi \neq \mathbf{0}$, then $\|\cdot\|_{[\psi]}$ is a norm on the vector space $A S^{1}(\mathbb{R})$ that is invariant under reparametrization.

Note. In the rest of the paper the equality $\|\varphi\|_{[\psi]}=\|\psi\|_{[\varphi]}$ will be called the exchange property.

Proof. (See Figure [6.) First of all, let us prove that if $\psi \neq \mathbf{0}$, then $\|\cdot\|_{[\psi]}$ is a norm. Clearly $\|\varphi\|_{[\psi]}$ is a non-negative real number. Indeed, the finiteness of the sup follows from Lemma 3.1ii) and the invariance of the total variation under reparametrizations. Moreover, it holds that $\|\lambda \varphi\|_{[\psi]}=|\lambda| \cdot\|\varphi\|_{[\psi]}$ for any $\lambda \in \mathbb{R}$, and the triangle inequality is easily verified. Also, if $\varphi \equiv 0$, then obviously $\|\varphi\|_{[\psi]}=0$. Therefore, the only thing we have to prove is that $\|\varphi\|_{[\psi]}=0$ implies $\varphi \equiv 0$. We prove this statement by contradiction, assuming that $\|\varphi\|_{[\psi]}=0$ and $\varphi^{*}\left(t_{0}\right) \neq 0$ for some $t_{0}$. Let $[a, b]$ be a bounded closed interval $(a \neq b)$ containing the compact support of $\frac{d \varphi^{*}}{d t}$ so that $\varphi^{*}(t)=0$ for $t \geq b$. Analogously, define $[\alpha, \beta]$ to be a bounded closed interval $(\alpha \neq \beta)$ containing the compact support of $\frac{d \psi}{d t}$, so that $\psi(t)=0$ for $t \leq \alpha$. Since $\psi$ is not constant, a point $t_{1}$ exists with $\psi\left(t_{1}\right) \neq 0$. It is easy to see that for every $\varepsilon>0$ an orientation-preserving $C^{1}$-diffeomorphism $h_{\varepsilon}: \mathbb{R} \rightarrow \mathbb{R}$ exists such that

i): $h_{\varepsilon}\left(\left[t_{0}-\varepsilon, t_{0}+\varepsilon\right]\right)=\left[\alpha, t_{1}\right]$;

ii): $\frac{d h_{\varepsilon}}{d t}(t)=\varepsilon$ for $t \geq t_{0}+\varepsilon$.

We define $\hat{\psi}=\psi \circ h_{\varepsilon}$. Obviously, $\hat{\psi} \in A S^{1}(\mathbb{R})$ and $\hat{\psi} \sim \psi$. Note that $\left|\frac{d \hat{\psi}}{d t}(t)\right| \leq$ $\varepsilon \cdot \max \left|\frac{d \psi}{d t}\right|$ for $t \geq t_{0}+\varepsilon$, and that $\frac{d \hat{\psi}}{d t}(t)=0$ for $t \leq t_{0}-\varepsilon$ (since $\frac{d \psi}{d t}(t)=0$ for $t \leq \alpha)$. Taking $\varepsilon$ small enough, and remembering that $\varphi^{*}(t)=0$ for $t \geq b$, we easily get

$$
\begin{aligned}
& \left|\int_{-\infty}^{+\infty} \varphi^{*}(t) \cdot \frac{d \hat{\psi}}{d t}(t) d t\right|=\left|\int_{t_{0}-\varepsilon}^{b} \varphi^{*}(t) \cdot \frac{d \hat{\psi}}{d t}(t) d t\right| \\
& \geq\left|\int_{t_{0}-\varepsilon}^{t_{0}+\varepsilon} \varphi^{*}(t) \cdot \frac{d \hat{\psi}}{d t}(t) d t\right|-\max |\varphi| \cdot \varepsilon \cdot \max \left|\frac{d \psi}{d t}\right| \cdot\left(b-\left(t_{0}+\varepsilon\right)\right) .
\end{aligned}
$$


Now we observe that

$\left|\int_{t_{0}-\varepsilon}^{t_{0}+\varepsilon} \varphi^{*}(t) \cdot \frac{d \hat{\psi}}{d t}(t) d t-\int_{t_{0}-\varepsilon}^{t_{0}+\varepsilon} \varphi^{*}\left(t_{0}\right) \cdot \frac{d \hat{\psi}}{d t}(t) d t\right| \leq \max _{\left[t_{0}-\varepsilon, t_{0}+\varepsilon\right]}\left|\varphi^{*}(t)-\varphi^{*}\left(t_{0}\right)\right| \cdot V_{\psi}$.

Since we know that $\int_{t_{0}-\varepsilon}^{t_{0}+\varepsilon} \varphi^{*}\left(t_{0}\right) \cdot \frac{d \hat{\psi}}{d t}(t) d t=\varphi^{*}\left(t_{0}\right) \cdot \hat{\psi}\left(t_{0}+\varepsilon\right)=\varphi^{*}\left(t_{0}\right) \cdot \psi\left(t_{1}\right) \neq 0$, it follows that $\lim _{\varepsilon \rightarrow 0^{+}} \int_{t_{0}-\varepsilon}^{t_{0}+\varepsilon} \varphi^{*}(t) \cdot \frac{d \hat{\psi}}{d t}(t) d t=\varphi^{*}\left(t_{0}\right) \cdot \psi\left(t_{1}\right) \neq 0$. Therefore the value $\left|\int_{-\infty}^{+\infty} \varphi^{*}(t) \cdot \frac{d \hat{\psi}}{d t}(t) d t\right|$ is positive if we have chosen a small enough $\varepsilon$ in the construction of $h_{\varepsilon}$. Hence $\|\varphi\|_{[\psi]}>0$, against our assumption. So we have proved that $\|\cdot\|_{[\psi]}$ is a norm.

Now let us consider a diffeomorphism $h \in D_{+}^{1}(\mathbb{R})$ and prove that $\|\varphi \circ h\|_{[\psi]}=$ $\|\varphi\|_{[\psi]}$; i.e., $\|\cdot\|_{[\psi]}$ is invariant under reparametrization. Setting $\tau=-h(-t)$, and $\hat{h}(\tau)=-h^{-1}(-\tau)=t$, since $\hat{h} \in D_{+}^{1}(\mathbb{R})$ we obtain that

$$
\begin{aligned}
\|\varphi \circ h\|_{[\psi]} & =\sup _{\hat{\psi} \in[\psi]}\left|\int_{-\infty}^{+\infty}(\varphi \circ h)(-t) \cdot \frac{d \hat{\psi}}{d t}(t) d t\right| \\
& =\sup _{\hat{\psi} \in[\psi]}\left|\int_{-\infty}^{+\infty} \varphi^{*}(\tau) \cdot \frac{d \hat{\psi}}{d \tau}(\hat{h}(\tau)) \cdot \frac{d \hat{h}}{d \tau}(\tau) d \tau\right| \\
& =\sup _{\hat{\psi} \in[\psi]}\left|\int_{-\infty}^{+\infty} \varphi^{*}(\tau) \cdot \frac{d(\hat{\psi} \circ \hat{h})}{d \tau}(\tau) d \tau\right| \\
& =\sup _{\hat{\psi} \in[\psi]}\left|\int_{-\infty}^{+\infty} \varphi^{*}(\tau) \cdot \frac{d \hat{\psi}}{d \tau}(\tau) d \tau\right|=\|\varphi\|_{[\psi]} .
\end{aligned}
$$

The equality $\|\varphi\|_{[\psi]}=\|\varphi\|_{[-\psi]}$ is trivial.

The equality $\|\varphi\|_{[\psi]}=\|\psi\|_{[\varphi]}$ follows from Lemma 3.1i), by observing that

$$
\begin{gathered}
\|\varphi\|_{[\psi]}=\sup _{h \in D_{+}^{1}(\mathbb{R})}\left|\int_{-\infty}^{+\infty} \varphi^{*}(t) \cdot \frac{d(\psi \circ h)}{d t}(t) d t\right| \\
=\sup _{h \in D_{+}^{1}(\mathbb{R})}\left|\int_{-\infty}^{+\infty} \frac{d \varphi^{*}}{d t}(t) \cdot \psi(h(t)) d t\right|=\sup _{h \in D_{+}^{1}(\mathbb{R})}\left|\int_{-\infty}^{+\infty} \frac{d \varphi}{d t}(t) \cdot \psi(h(-t)) d t\right| \\
=\sup _{\hat{h} \in D_{+}^{1}(\mathbb{R})}\left|\int_{-\infty}^{+\infty} \frac{d \varphi}{d \tau}(\hat{h}(\tau)) \cdot \psi^{*}(\tau) \cdot \frac{d \hat{h}}{d \tau}(\tau) d \tau\right| \\
=\sup _{\hat{h} \in D_{+}^{1}(\mathbb{R})}\left|\int_{-\infty}^{+\infty} \frac{d(\varphi \circ \hat{h})}{d t}(\tau) \cdot \psi^{*}(\tau) d \tau\right|=\|\psi\|_{[\varphi]},
\end{gathered}
$$

where, once again, $\tau=-h(-t)$ and $t=\hat{h}(\tau)=-h^{-1}(-\tau)$. 
The inequality $\|\varphi\|_{[\psi]} \leq \min \left\{\max |\varphi| \cdot V_{\psi}, \max |\psi| \cdot V_{\varphi}\right\}$ follows from Lemma 3.1ii) and the equality $\|\varphi\|_{[\psi]}=\|\psi\|_{[\varphi]}$.

In what follows, the norms $\|\cdot\|_{[\psi]}(\psi \neq \mathbf{0})$ will be called standard reparametrization invariant norms (or standard RPI-norms).

3.2. Two examples of standard RPI-norms. A simple instance of a standard RPI-norm is given by the $L_{\infty}$-norm, as the following proposition states.

Proposition 3.3. $\|\varphi\|_{[S]}=\max |\varphi|$.

Proof. When $\varphi=\mathbf{0}$, the claim is trivial, so let us assume $\max |\varphi| \neq 0$. By Theorem 3.2. $\|\varphi\|_{[S]} \leq \max |\varphi|$. Let $t_{\max }$ now be a value for which $\left|\varphi^{*}\left(t_{\max }\right)\right|=$ $\max |\varphi|$ and consider, for $\varepsilon>0$, the function $S_{\varepsilon}(t)=S\left(\frac{t-t_{\max }}{\varepsilon}\right)$. Obviously, $S_{\varepsilon} \sim S$. We observe that $\frac{d S_{\varepsilon}}{d t}$ vanishes outside the interval $\left[t_{\max }-\varepsilon, t_{\max }+\varepsilon\right]$ and $\int_{t_{\max }-\varepsilon}^{t_{\max }+\varepsilon} \frac{d S_{\varepsilon}}{d t}(t) d t=S_{\varepsilon}\left(t_{\max }+\varepsilon\right)-S_{\varepsilon}\left(t_{\max }-\varepsilon\right)=1$. For any sufficiently small $\varepsilon$, we have that $\varphi^{*}(t)$ has constant non-zero sign in $\left[t_{\max }-\varepsilon, t_{\max }+\varepsilon\right]$. So, it follows that

$$
\begin{aligned}
\max & |\varphi|-\left|\int_{-\infty}^{+\infty} \varphi^{*}(t) \cdot \frac{d S_{\varepsilon}}{d t}(t) d t\right| \\
& =\max |\varphi|-\left|\int_{t_{\max }-\varepsilon}^{t_{\max }+\varepsilon} \varphi^{*}(t) \cdot \frac{d S_{\varepsilon}}{d t}(t) d t\right| \\
& =\int_{t_{\max }-\varepsilon}^{t_{\max }+\varepsilon} \max |\varphi| \cdot \frac{d S_{\varepsilon}}{d t}(t) d t-\int_{t_{\max }-\varepsilon}^{t_{\max }+\varepsilon}\left|\varphi^{*}(t)\right| \cdot \frac{d S_{\varepsilon}}{d t}(t) d t \\
& =\int_{t_{\max }-\varepsilon}^{t_{\max }+\varepsilon}\left(\max |\varphi|-\left|\varphi^{*}(t)\right|\right) \cdot \frac{d S_{\varepsilon}}{d t}(t) d t \\
& \leq \int_{t_{\max }-\varepsilon}^{t_{\max }+\varepsilon}\left(\max |\varphi|-\min _{\left|t-t_{\max }\right| \leq \varepsilon}\left|\varphi^{*}(t)\right|\right) \cdot \frac{d S_{\varepsilon}}{d t}(t) d t \\
& =\left(\max |\varphi|-\min _{\left|t-t_{\max }\right| \leq \varepsilon}\left|\varphi^{*}(t)\right|\right) \cdot \int_{t_{\max }-\varepsilon}^{t_{\max }+\varepsilon} \frac{d S_{\varepsilon}}{d t}(t) d t \\
& =\max |\varphi|-\min _{\left|t-t_{\max }\right| \leq \varepsilon}\left|\varphi^{*}(t)\right| .
\end{aligned}
$$

The continuity of $\varphi$ implies that $\lim _{\varepsilon \rightarrow 0^{+}} \min _{\left|t-t_{\max }\right| \leq \varepsilon}\left|\varphi^{*}(t)\right|=\varphi^{*}\left(t_{\max }\right)=$ $\max |\varphi|$. Hence the equality $\lim _{\varepsilon \rightarrow 0^{+}}\left|\int_{-\infty}^{+\infty} \varphi^{*}(t) \cdot \frac{d S_{\varepsilon}}{d t}(t) d t\right|=\max |\varphi|$ holds. Since we have already seen that $\|\varphi\|_{[S]} \leq \max |\varphi|$, this proves that $\|\varphi\|_{[S]}=\max |\varphi|$.

Another simple standard RPI-norm on $A S^{1}(\mathbb{R})$ is given by $\max \varphi-\min \varphi$, as the following proposition states.

Proposition 3.4. $\|\varphi\|_{[\Lambda]}=\max \varphi-\min \varphi$.

Proof. Let us take a $C^{1}$-diffeomorphism $h \in D_{+}^{1}(\mathbb{R})$. Possibly by substituting $\varphi$ with $-\varphi$ we can assume that $\int_{-\infty}^{+\infty} \varphi^{*}(t) \cdot \frac{d(\Lambda \circ h)}{d t}(t) d t \geq 0$. Hence, by recalling that 

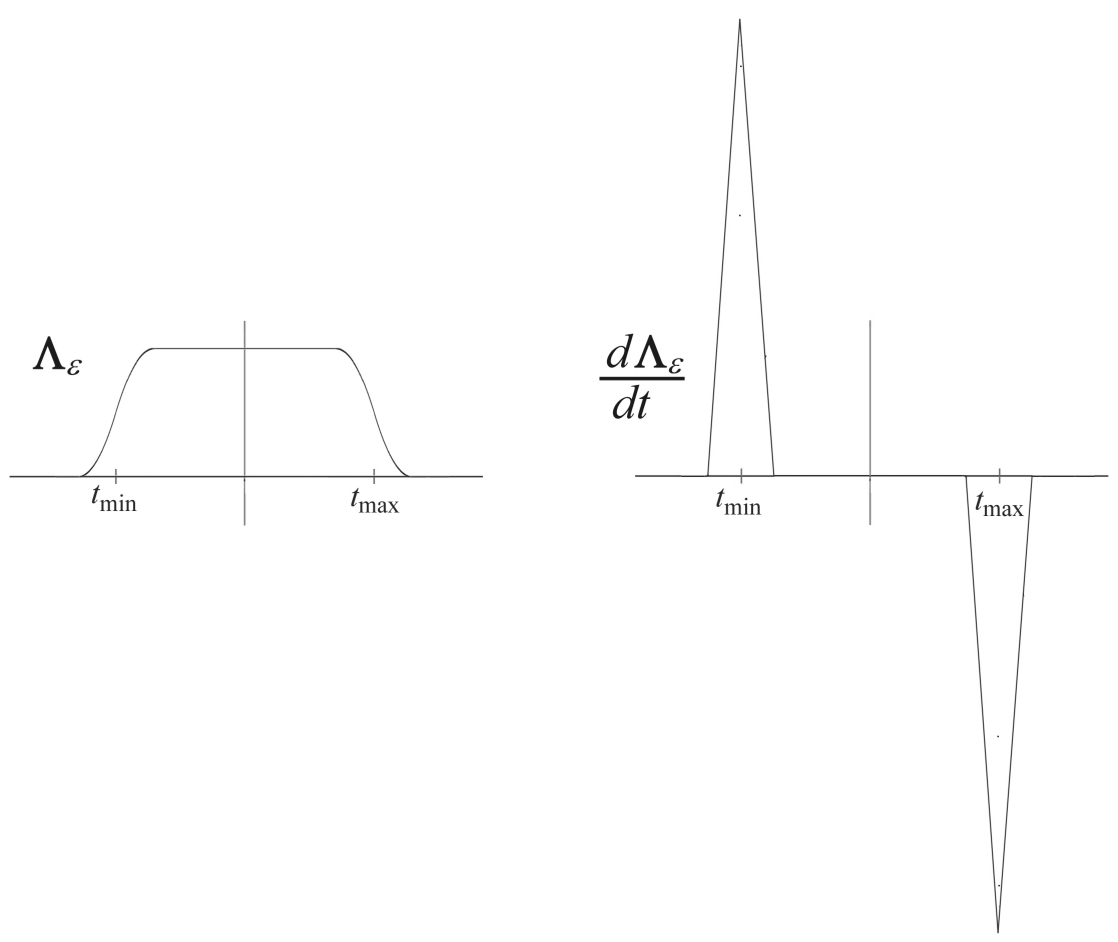

Figure 7. The function $\Lambda_{\varepsilon}$ used in the proof of Proposition 3.4 and its derivative (case $t_{\min }<t_{\max }$ ).

$\Lambda$ is increasing in $(-\infty, 0]$ and decreasing in $[0,-\infty)$, we obtain that

$$
\begin{aligned}
& \left|\int_{-\infty}^{+\infty} \varphi^{*}(t) \cdot \frac{d(\Lambda \circ h)}{d t}(t) d t\right| \\
& \quad=\int_{-\infty}^{+\infty} \varphi^{*}(t) \cdot \frac{d(\Lambda \circ h)}{d t}(t) d t \\
& \quad=\int_{-\infty}^{h^{-1}(0)} \varphi^{*}(t) \cdot \frac{d(\Lambda \circ h)}{d t}(t) d t+\int_{h^{-1}(0)}^{+\infty} \varphi^{*}(t) \cdot \frac{d(\Lambda \circ h)}{d t}(t) d t \\
& \quad \leq \int_{-\infty}^{h^{-1}(0)} \max \varphi \cdot \frac{d(\Lambda \circ h)}{d t}(t) d t+\int_{h^{-1}(0)}^{+\infty} \min \varphi \cdot \frac{d(\Lambda \circ h)}{d t}(t) d t \\
& \quad=\max \varphi \cdot\left(\Lambda(0)-\lim _{t \rightarrow-\infty} \Lambda(t)\right)+\min \varphi \cdot\left(\lim _{t \rightarrow+\infty} \Lambda(t)-\Lambda(0)\right) \\
& \quad=\max \varphi-\min \varphi .
\end{aligned}
$$

Since $\|\varphi\|_{[\Lambda]}=\|-\varphi\|_{[\Lambda]}$, it follows that $\|\varphi\|_{[\Lambda]} \leq \max \varphi-\min \varphi$.

Let $t_{\min }$ and $t_{\max }$ be a minimum point and a maximum point for $\varphi$, respectively. If $t_{\min }=t_{\max }$, then $\varphi \equiv 0$ and our statement is trivial. So, let us assume that $t_{\min } \neq t_{\max }$. Let us define $t_{0}=\min \left\{t_{\min }, t_{\max }\right\}$ and $t_{1}=\max \left\{t_{\min }, t_{\max }\right\}$. We consider the function $\Lambda_{\varepsilon}=S\left(\frac{t-t_{0}}{\varepsilon}\right)-S\left(\frac{t-t_{1}}{\varepsilon}\right)$ (see Figure 7). Even if $\Lambda_{\varepsilon}$ 
is not equivalent to $\Lambda$, we have that $\|\varphi\|_{[\Lambda]}=\|\varphi\|_{\left[\Lambda_{\varepsilon}\right]}$ for any small enough $\varepsilon$. Indeed, from Theorem 3.2 (exchange property) and Proposition 2.15, it follows that $\|\varphi\|_{[\Lambda]}=\|\Lambda\|_{[\varphi]}=\left\|\Lambda_{\varepsilon}\right\|_{[\varphi]}=\|\varphi\|_{\left[\Lambda_{\varepsilon}\right]}$. Since it is easy to verify that the equality $\lim _{\varepsilon \rightarrow 0^{+}}\left|\int_{-\infty}^{+\infty} \varphi^{*}(t) \frac{d \Lambda_{\varepsilon}}{d t}(t) d t\right|=\max \varphi-\min \varphi$ holds, this implies that $\|\varphi\|_{[\Lambda]} \geq \max \varphi-\min \varphi$. Therefore our statement follows.

3.2.1. The key idea in using standard RPI-norms. The two examples seen in the previous section show that, in some sense, computing standard RPI-norms is equivalent to computing the absolute value of a linear combination of Dirac deltas, applied to the function $\varphi^{*}(t)$. Indeed, it is easy to verify that in order to get $\|\varphi\|_{[S]}$ and $\|\varphi\|_{[\Lambda]}$ we have to compute the values $\sup _{t}\left|\delta_{t}\left(\varphi^{*}\right)\right|$ and $\sup _{t_{0} \leq t_{1}}\left|\delta_{t_{0}}\left(\varphi^{*}\right)-\delta_{t_{1}}\left(\varphi^{*}\right)\right|$, where $\delta_{t}$ is the usual Dirac delta at point $t$. The "weights" of the Dirac deltas are determined by the integral $\int_{-1}^{1} \frac{d S}{d t}(t) d t=1$ in the first case, and by the integrals $\int_{-2}^{0} \frac{d \Lambda}{d t}(t) d t=1, \int_{0}^{2} \frac{d \Lambda}{d t}(t) d t=-1$ in the latter, i.e. the integrals of $\frac{d \psi}{d t}$ on the maximal intervals where the derivative of the function $\psi$ defining the norm $\|\cdot\|_{[\psi]}$ does not vanish. In order to compute $\|\varphi\|_{[S]}$ we place $\delta_{t}$ at a point where $|\varphi|$ takes its maximum value, while, when we compute $\|\varphi\|_{[\Lambda]}$, we place $\delta_{t_{0}}$ and $\delta_{t_{1}}$ at the points where $\varphi$ takes its maximum value and its minimum value (not necessarily in this order). We shall carefully analyze and generalize this approach in Section 4

3.3. Not every RPI-norm is a standard RPI-norm. RPI-norms are not necessarily standard RPI-norms. In order to show this, now we give a useful property of standard RPI-norms.

Proposition 3.5. Let $\|\cdot\|$ be an RPI-norm. If it can be obtained as a finite linear combination of standard RPI-norms with positive coefficients, then $\|S\| \leq\|\Lambda\|$.

Proof. Let us assume that some functions $\psi_{1}, \ldots, \psi_{k} \in A S^{1}(\mathbb{R})$ and a $k$-tuple $\left(a_{1}, \ldots, a_{k}\right)$ of positive numbers exist such that, for every $\varphi \in A S^{1}(\mathbb{R})$, it holds that $\|\varphi\|=\sum_{i=1}^{k} a_{i} \cdot\|\varphi\|_{\left[\psi_{i}\right]}$. Let us consider the functions $\tilde{\psi}_{1}, \ldots, \tilde{\psi}_{k}$, such that, for $i=1, \ldots, k$, if $\max \left|\psi_{i}\right|=\max \psi_{i}$, then $\tilde{\psi}_{i}=\psi_{i}$; otherwise $\tilde{\psi}_{i}=-\psi_{i}$. By Theorem 3.2, it holds that $\|\varphi\|=\sum_{i=1}^{k} a_{i} \cdot\|\varphi\|_{\left[\tilde{\psi}_{i}\right]}$. Therefore, by the exchange property and Proposition 3.3 , we have that

$$
\|S\|=\sum_{i=1}^{k} a_{i} \cdot\|S\|_{\left[\tilde{\psi}_{i}\right]}=\sum_{i=1}^{k} a_{i} \cdot\left\|\tilde{\psi}_{i}\right\|_{[S]}=\sum_{i=1}^{k} a_{i} \cdot \max \left|\tilde{\psi}_{i}\right|=\sum_{i=1}^{k} a_{i} \cdot \max \tilde{\psi}_{i} .
$$

Analogously, by the exchange property and Proposition 3.4, we obtain that $\|\Lambda\|=$ $\sum_{i=1}^{k} a_{i} \cdot\left(\max \tilde{\psi}_{i}-\min \tilde{\psi}_{i}\right)$. The claim immediately follows since $\sum_{i=1}^{k} a_{i} \cdot \min \tilde{\psi}_{i} \leq 0$ (recall that $\min \psi \leq 0$ for every $\psi \in A S^{1}(\mathbb{R})$ ).

As a consequence of this property, we can furnish an example of an RPI-norm that cannot be represented as a linear combination with positive coefficients of standard RPI-norms.

Corollary 3.6. The RPI-norm $\|\varphi\|=\max |\varphi|+\lim _{t \rightarrow+\infty}|\varphi(t)|$ cannot be represented as a finite linear combination with positive coefficients of standard RPInorms. In particular, it is not a standard RPI-norm.

Proof. It is sufficient to observe that $\|S\|=\max |S|+\lim _{t \rightarrow+\infty}|S(t)|=2,\|\Lambda\|=$ $\max |\Lambda|+\lim _{t \rightarrow+\infty}|\Lambda(t)|=1$, and apply Proposition 3.5. 
Remark 3.7. It could be interesting to know whether the norm $\max |\varphi|+$ $\lim _{t \rightarrow+\infty}|\varphi(t)|$ can be represented either as a sup or as an inf of a suitable set of standard RPI-norms.

Another example of an RPI-norm that cannot be expressed as a finite linear combination with positive coefficients of standard RPI-norms is the total variation.

Proposition 3.8. The total variation cannot be represented as a finite linear combination with positive coefficients of standard RPI-norms. In particular, it is not a standard RPI-norm.

Proof. If the total variation could be represented as a linear combination with positive coefficients of standard RPI-norms, the equality $V_{\varphi}=\sum_{i=1}^{k} a_{i} \cdot\|\varphi\|_{\left[\psi_{i}\right]}$ would hold for every $\varphi \in A S^{1}(\mathbb{R})$ when a suitable set $\left\{a_{1}, \ldots, a_{k}\right\}$ of positive coefficients is chosen.

By Theorem 3.2 we would have that $V_{\varphi} \leq \sum_{i=1}^{k} a_{i} V_{\psi_{i}} \cdot \max |\varphi|$. This inequality contradicts the fact that we can easily find a function $\bar{\varphi} \in A S^{1}(\mathbb{R})$ such that $\max \bar{\varphi} \neq 0$ and the ratio $\frac{V_{\bar{\varphi}}}{\max \bar{\varphi}}$ is arbitrarily large.

Nevertheless, the total variation can be seen as the sup of a suitable set of standard RPI-norms, as shown in the following Section 3.3.1

3.3.1. The total variation is a sup of standard RPI-norms. We have seen in Proposition 3.8 that the total variation is not a standard RPI-norm. We now show that it is the sup of a family of standard RPI-norms.

Proposition 3.9. For every $n \geq 1$, let us set $L_{n}(t)=\sum_{i=0}^{n-1}(-1)^{i} \Lambda(t-4 i)$. Then, for every $\varphi \in A S^{1}(\mathbb{R})$, we have that $V_{\varphi}=\sup _{n \in \mathbb{N}^{+}}\|\varphi\|_{\left[L_{n}\right]}=\lim _{n \rightarrow \infty}\|\varphi\|_{\left[L_{n}\right]}$.

Proof. Let us prove the first equality. By applying Theorem 3.2, we obtain that $\|\varphi\|_{\left[L_{n}\right]} \leq \max \left|L_{n}\right| \cdot V_{\varphi}=V_{\varphi}$. We only have to show that for every $\varepsilon>0$ an $n$ exists such that $V_{\varphi}-\|\varphi\|_{\left[L_{n}\right]} \leq \varepsilon$. This is trivially true if $\varphi=0$, so let us assume $\varphi \neq \mathbf{0}$. Let $[a, b]$ be a closed interval containing the support of $\frac{d \varphi^{*}}{d t}$. Let us recall that $V_{\varphi}=V_{\varphi^{*}}=\sup _{k} \sup _{a=t_{0}<t_{1}<\ldots<t_{k}=b} \sum_{i=0}^{k-1}\left|\varphi^{*}\left(t_{i+1}\right)-\varphi^{*}\left(t_{i}\right)\right|$. Hence, there exist $n \geq 1$ and a partition $a=\bar{\tau}_{0}<\bar{\tau}_{1}<\ldots<\bar{\tau}_{n}=b$ of $[a, b]$, such that

$$
0 \leq V_{\varphi}-\sum_{i=0}^{n-1}\left|\varphi^{*}\left(\bar{\tau}_{i+1}\right)-\varphi^{*}\left(\bar{\tau}_{i}\right)\right| \leq \frac{\varepsilon}{2} .
$$

Possibly by substituting our partition with a simpler one, we can assume that $\varphi^{*}\left(\bar{\tau}_{i+1}\right)-\varphi^{*}\left(\bar{\tau}_{i}\right) \neq 0$ for $i=0, \ldots, n-1$ and, for $n \geq 2, \operatorname{sign}\left(\varphi^{*}\left(\bar{\tau}_{i+1}\right)-\varphi^{*}\left(\bar{\tau}_{i}\right)\right) \neq$ $\operatorname{sign}\left(\varphi^{*}\left(\bar{\tau}_{i+2}\right)-\varphi^{*}\left(\bar{\tau}_{i+1}\right)\right)$ for $i=0, \ldots, n-2$.

For every sufficiently small $\eta>0$, let us consider an orientation-preserving $C^{1}$ diffeomorphism $h_{\eta}$ that takes the interval $\left[\bar{\tau}_{i}-\eta, \bar{\tau}_{i}+\eta\right]$ onto the interval $[4(i-1)+$ $\eta, 4 i-\eta]$, for every integer $i$ with $0 \leq i \leq n$. Let us observe that the function $L_{n}$ is monotone on every interval $[4(i-1)+\eta, 4 i-\eta]$ (cf. Figure 8).

For any small enough $\eta$, by recalling that $\varphi^{*}\left(\bar{\tau}_{n}\right)=\varphi^{*}(b)=0$, it is easy to prove that

$$
|| \varphi^{*}\left(\bar{\tau}_{0}\right)+\sum_{i=1}^{n-1}(-1)^{i} \cdot 2 \cdot \varphi^{*}\left(\bar{\tau}_{i}\right)|-| \int_{-\infty}^{+\infty} \varphi^{*}(\tau) \cdot \frac{d\left(L_{n} \circ h_{\eta}\right)}{d \tau}(\tau) d \tau|| \leq \frac{\varepsilon}{2} .
$$




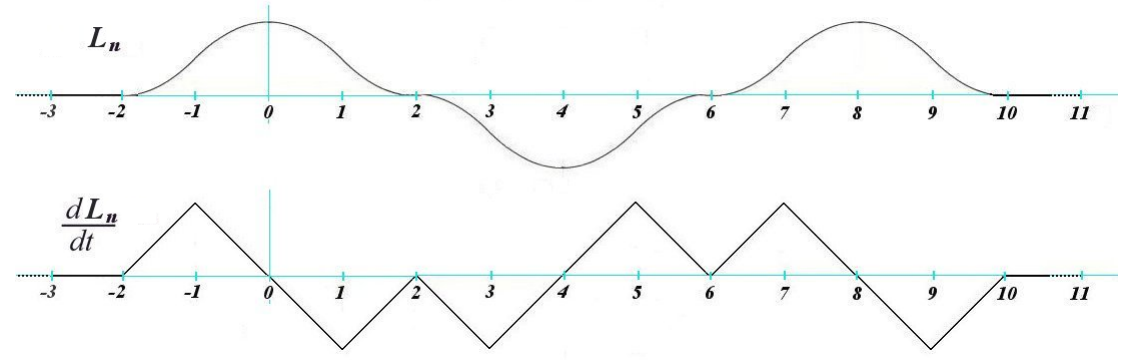

Figure 8. The function $L_{n}$ and its derivative (case $n=3$ ), used in Proposition 3.9.

The assumptions about the differences $\varphi^{*}\left(\bar{\tau}_{i+1}\right)-\varphi^{*}\left(\bar{\tau}_{i}\right)$ and, once again, the condition $\varphi^{*}\left(\bar{\tau}_{n}\right)=\varphi^{*}(b)=0$, imply that

$$
\sum_{i=0}^{n-1}\left|\varphi^{*}\left(\bar{\tau}_{i+1}\right)-\varphi^{*}\left(\bar{\tau}_{i}\right)\right|=\left|\varphi^{*}\left(\bar{\tau}_{0}\right)+\sum_{i=1}^{n-1}(-1)^{i} \cdot 2 \cdot \varphi^{*}\left(\bar{\tau}_{i}\right)\right| .
$$

It follows that

$$
\left|V_{\varphi}-\right| \int_{-\infty}^{+\infty} \varphi^{*}(\tau) \cdot \frac{d\left(L_{n} \circ h_{\eta}\right)}{d \tau}(\tau) d \tau|| \leq \varepsilon
$$

Hence $V_{\varphi}-\|\varphi\|_{\left[L_{n}\right]} \leq \varepsilon$, and the first equality in our statement is proved.

The second equality follows from the first by observing that the sequence $\left(\|\varphi\|_{\left[L_{n}\right]}\right)$ is increasing.

Remark 3.10. In plain words, our proof of Proposition 3.9 is based on recognizing that $\sum_{i=0}^{n-1}\left|\varphi^{*}\left(\bar{\tau}_{i+1}\right)-\varphi^{*}\left(\bar{\tau}_{i}\right)\right|$ can be seen as the value taken by the absolute value of the linear functional $\delta_{\bar{\tau}_{0}}+\sum_{i=1}^{n-1}(-1)^{i} 2 \cdot \delta_{\bar{\tau}_{i}}$ computed at $\varphi^{*}$, where $\delta_{t}$ is the usual Dirac delta at point $t$. The reparametrization $h_{\eta}$ allows us to approximate $\left|\delta_{\bar{\tau}_{0}}\left(\varphi^{*}\right)+\sum_{i=1}^{n-1}(-1)^{i} 2 \cdot \delta_{\bar{\tau}_{i}}\left(\varphi^{*}\right)\right|$ by $\left|\int_{-\infty}^{+\infty} \varphi^{*}(\tau) \cdot \frac{d\left(L_{n} \circ h_{\eta}\right)}{d \tau}(\tau) d \tau\right|$, by "concentrating" at $\bar{\tau}_{0}$ and at the other $\bar{\tau}_{i}$ 's a signed variation of $L_{n} \circ h_{\eta}$ approximately equal to 1 and \pm 2 , respectively. This last idea will be developed in Section 4 by using general weights (not just 1 and \pm 2 ) for the Dirac deltas, and its generalization will lead to the Representation Theorem 4.16 This result assures that every standard RPI-norm can be seen as the absolute value of a suitable linear combination of Dirac deltas, maximized with respect to the movements that preserve the deltas' position order. We could obtain Proposition 3.9 as a consequence of the Representation Theorem, but we have preferred to anticipate this result for the sake of clarity of exposition. Moreover, this choice allows us to illustrate the ideas that we are going to develop.

\section{Discrete REPRESEnTATION OF StANDARD RPI-NORMS}

In this section we show how to compute the standard RPI-norms in a simpler, discrete way.

As we have seen in Section 3.2 the standard RPI-norms $\max |\varphi|$ and $\max \varphi-$ $\min \varphi$ can be expressed $\operatorname{asup}_{t}\left|\delta_{t}\left(\varphi^{*}\right)\right|$ and $\sup _{t_{1} \leq t_{2}}\left|\delta_{t_{1}}\left(\varphi^{*}\right)-\delta_{t_{2}}\left(\varphi^{*}\right)\right|$ respectively, where $\delta_{t}$ is the usual Dirac delta at point $t$. 

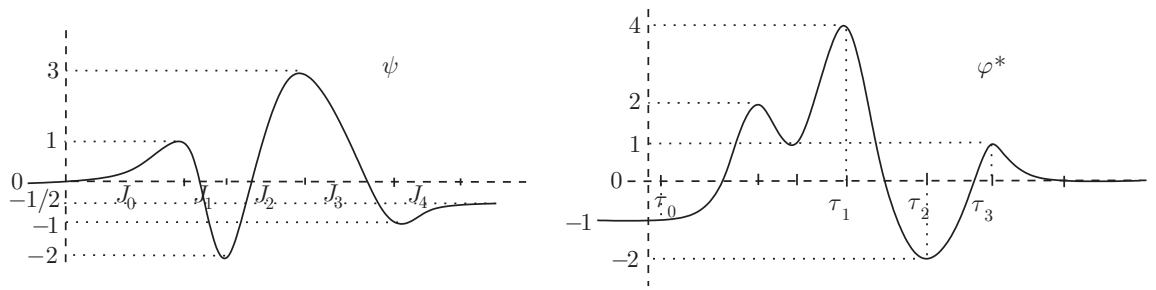

Figure 9. The functions used in the example described in Section 4

The fact that they can be seen as the sup of the absolute value of linear combinations of Dirac deltas is a general property of standard RPI-norms. The basic idea underlying this fact is that the sup of $\left|\int_{-\infty}^{+\infty} \varphi^{*}(t) \cdot \frac{d(\psi \circ h)}{d t}(t) d t\right|$ for $h \in D_{+}^{1}(\mathbb{R})$ is obtained by considering a sequence of reparametrizations increasingly concentrating the variation of $\psi$ at suitable points. Passing from the integral definition of the standard RPI-norm to linear combinations of Dirac deltas, the sup with respect to orientation-preserving reparametrizations is replaced by a sup with respect to movements that shift the Dirac delta centers without changing their order (Theorem 4.9). We shall see that the best choice is to place these Dirac delta centers at critical points of $\varphi$ (Representation Theorem 4.16).

By way of exemplification, let us consider the norm $\|\varphi\|_{[\psi]}$ where $\psi(t)$ and $\varphi^{*}(t)$ are the functions illustrated in Figure 9. Let us denote by $J_{i}=\left(a_{i}, b_{i}\right)$ the maximal open intervals where the derivative of $\psi$ has a constant non-zero sign. It holds that $\psi\left(b_{0}\right)-\psi\left(a_{0}\right)=1, \psi\left(b_{1}\right)-\psi\left(a_{1}\right)=-3, \psi\left(b_{2}\right)-\psi\left(a_{2}\right)=5, \psi\left(b_{3}\right)-\psi\left(a_{3}\right)=-4$, $\psi\left(b_{4}\right)-\psi\left(a_{4}\right)=1 / 2$. In order to increase the value $\left|\int_{-\infty}^{\infty} \varphi^{*}(t) \cdot \frac{d(\psi \circ h)}{d t}(t) d t\right|$, it is convenient to take reparametrizations $h_{\eta}$ that transform smaller and smaller neighbourhoods of suitable points $t_{0} \leq t_{1} \leq t_{2} \leq t_{3} \leq t_{4}$ ordinately to the intervals $\left(a_{0}+\eta, b_{0}-\eta\right),\left(a_{1}+\eta, b_{1}-\eta\right), \ldots,\left(a_{4}+\eta, b_{4}-\eta\right)$, with a smaller and smaller $\eta>0$ (or to $\left(a_{0}+\eta, b_{1}-\eta\right)$ if, say, $t_{0}=t_{1}$, and so on). By passing to the limit, we obtain that

$$
\begin{aligned}
\sup _{h} & \left|\int_{-\infty}^{\infty} \varphi^{*}(t) \cdot \frac{d(\psi \circ h)}{d t}(t) d t\right| \\
& =\sup _{t_{0} \leq t_{1} \leq t_{2} \leq t_{3} \leq t_{4}}\left|1 \cdot \varphi^{*}\left(t_{0}\right)-3 \cdot \varphi^{*}\left(t_{1}\right)+5 \cdot \varphi^{*}\left(t_{2}\right)-4 \cdot \varphi^{*}\left(t_{3}\right)+\frac{1}{2} \cdot \varphi^{*}\left(t_{4}\right)\right| .
\end{aligned}
$$

In other words,

$$
\begin{aligned}
\sup _{h} & \left|\int_{-\infty}^{\infty} \varphi^{*}(t) \cdot \frac{d(\psi \circ h)}{d t}(t) d t\right| \\
& =\sup _{t_{0} \leq t_{1} \leq t_{2} \leq t_{3} \leq t_{4}}\left|\left(1 \cdot \delta_{t_{0}}-3 \cdot \delta_{t_{1}}+5 \cdot \delta_{t_{2}}-4 \cdot \delta_{t_{3}}+\frac{1}{2} \cdot \delta_{t_{4}}\right)\left(\varphi^{*}\right)\right| .
\end{aligned}
$$

Now we easily see that, in order to get the greatest value, the $t_{i}$ 's must be critical points of $\varphi^{*}$. In particular, in this case the sup is attained when $t_{0}=t_{1}=\tau_{0}$, $t_{2}=\tau_{1}, t_{3}=\tau_{2}, t_{4}=\tau_{3}$, so that $\|\varphi\|_{[\psi]}=30.5$.

We point out that the linear combinations considered can involve infinitely many terms. This is the main difficulty to manage in this section and will require some computations. 
The key result obtained in this section (Representation Theorem 4.16) will be fundamental in the next section, where we shall use it to prove that all the standard RPI-norms of a piecewise monotone $C^{1}$-function $\varphi$ with compact support are sufficient to reconstruct $\varphi$, up to reparametrization, sign and an arbitrarily small error with respect to the total variation norm.

The first step to get these results is defining a bilinear function $F$ that will be useful in the sequel.

4.1. The functional $F$. In the remainder of Section 4 we shall assume that two functions $\varphi, \psi \in A S^{1}(\mathbb{R})$ are given, with $\psi \neq \mathbf{0}$. Let us consider the countable set $\mathcal{J}(\psi)$ of all maximal open intervals of $\mathbb{R}$ where $\frac{d \psi}{d t}$ does not vanish. We shall set $\mathcal{J}(\psi)=\left\{J_{i}\right\}_{i \in I}$, where $I$ is either the finite set $\{0, \ldots, n-1\}$ or the set $\mathbb{N}$. For each $J_{i}=\left(a_{i}, b_{i}\right) \in \mathcal{J}(\psi)$ we shall assume that a point $t_{i} \in\left[a_{i}, b_{i}\right]$ is chosen, such that

(1) $t_{i}$ is a point where the restriction of $\varphi^{*}$ to the closed interval $\left[a_{i}, b_{i}\right]$ takes its maximum value if $\left.\psi\right|_{a_{i}} ^{b_{i}}>0$;

(2) $t_{i}$ is a point where the restriction of $\varphi^{*}$ to the closed interval $\left[a_{i}, b_{i}\right]$ takes its minimum value if $\left.\psi\right|_{a_{i}} ^{b_{i}}<0$.

Here we set $\left.\psi\right|_{a_{i}} ^{b_{i}}=\psi\left(b_{i}\right)-\psi\left(a_{i}\right)$ (see example in Figure 10).

Definition 4.1. The set $\left\{t_{i}\right\}_{i \in I}$ is said to be a set of basepoints for the pair $(\varphi, \psi)$.

On the set $\mathcal{J}(\psi)$ we shall consider the order $\preceq$ induced by the $a_{i}$ 's. In other words, we shall set $J_{i} \preceq J_{j}$ if and only if $a_{i} \leq a_{j}$. This order will not need to coincide with the order induced by the index $i$. The symbol $\sigma_{i}$ will denote the sign of $\frac{d \psi}{d t}$ in the interval $J_{i}$, i.e. $\sigma_{i}=\left.\operatorname{sign} \psi\right|_{a_{i}} ^{b_{i}}$.

Definition 4.2. We define the bilinear functional $F: A S^{1}(\mathbb{R}) \times\left(A S^{1}(\mathbb{R})-\mathbf{0}\right) \rightarrow \mathbb{R}$ by setting

$$
F(\varphi, \psi)=\left.\sum_{i \in I} \psi\right|_{a_{i}} ^{b_{i}} \cdot \varphi^{*}\left(t_{i}\right)
$$

where $\left\{t_{i}\right\}_{i \in I}$ is a set of basepoints for $(\varphi, \psi)$.

In other words, $F(\varphi, \psi)=\left.\sum_{i \in I} \psi\right|_{a_{i}} ^{b_{i}} \cdot \delta_{t_{i}}\left(\varphi^{*}\right)$, where $\delta_{t_{i}}$ is the usual Dirac delta at point $t_{i}$. Note that the definition of $F(\varphi, \psi)$ does not depend on the particular choice of the set of basepoints for the pair $(\varphi, \psi)$. The idea underlying the definition of basepoint is to maximize each addend $\left.\psi\right|_{a_{i}} ^{b_{i}} \cdot \varphi^{*}(t)$ in the definition of $F$, when $t$ varies in $\left[a_{i}, b_{i}\right]$.

We observe that $|F(\varphi, \psi)| \leq \max |\varphi| \cdot V_{\psi}<+\infty$, since $\psi$ has bounded variation.

Remark 4.3. It is easy to verify that $\left\{t_{i}\right\}_{i \in I}$ is a set of basepoints for $(\varphi, \psi)$ if and only if for every $i \in I$,

$$
\varphi^{*}\left(t_{i}\right)=\sigma_{i} \cdot \max _{\left[a_{i}, b_{i}\right]}\left\{\sigma_{i} \cdot \varphi^{*}\right\} .
$$

Therefore, we get this equivalent definition for $F$ :

$$
F(\varphi, \psi)=\left.\sum_{i \in I} \sigma_{i} \cdot \psi\right|_{a_{i}} ^{b_{i}} \cdot \max _{\left[a_{i}, b_{i}\right]}\left\{\sigma_{i} \cdot \varphi^{*}\right\}
$$

Moreover, we observe that each set of basepoints for $(\varphi, \psi)$ is contained in the compact support of $\frac{d \psi}{d t}$. 


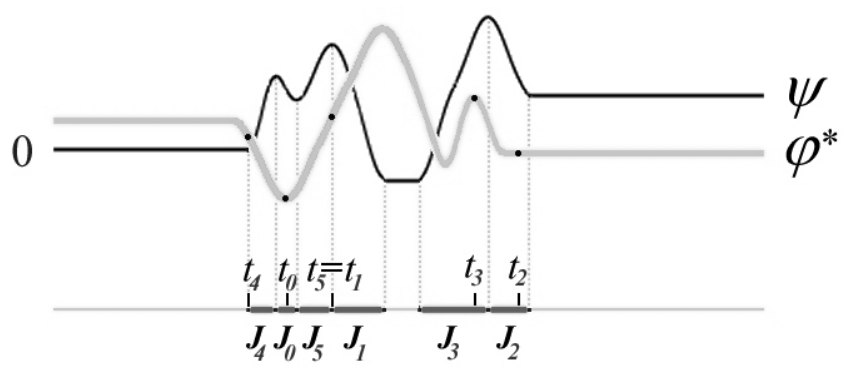

Figure 10. The set $\mathcal{J}(\psi)=\left\{J_{i}\right\}_{i \in I}$ for the displayed function $\psi$. A possible choice of the points $t_{i}$ is shown, with respect to $\varphi$ (the corresponding points on the graph of $\varphi^{*}$ are marked). Here we have $J_{4} \preceq J_{0} \preceq J_{5} \preceq J_{1} \preceq J_{3} \preceq J_{2}$. Observe that the order $\preceq$ in $\mathcal{J}(\psi)$ is not given by the index $i$, and that some $t_{i}$ 's belong to the boundary of the corresponding interval $J_{i}$.

4.2. Some useful properties of the functional $F$. Let us consider the set $H_{\psi}$ of all orientation-preserving $C^{1}$-diffeomorphisms of the real line that take each interval $J_{i} \in \mathcal{J}(\psi)$ to itself. The following lemma shows the key property of the functional $F$.

Lemma 4.4. $\sup _{h \in H_{\psi}}\left|\int_{-\infty}^{+\infty} \varphi^{*}(t) \cdot \frac{d(\psi \circ h)}{d t}(t) d t\right|=\max \{F(\varphi, \psi), F(\varphi,-\psi)\}$.

Proof. PART 1: $\sup _{h \in H_{\psi}}\left|\int_{-\infty}^{+\infty} \varphi^{*}(t) \cdot \frac{d(\psi \circ h)}{d t}(t) d t\right| \leq \max \{F(\varphi, \psi), F(\varphi,-\psi)\}$.

Let $\bar{\psi}=\psi \circ h$ with $h \in H_{\psi}$. Consider the countable set $\mathcal{J}(\bar{\psi})$ of all maximal open intervals of $\mathbb{R}$ where $\frac{d \bar{\psi}}{d t}$ does not vanish. Obviously, $\mathcal{J}(\bar{\psi})=\mathcal{J}(\psi)$ and $\operatorname{sign} \frac{d \bar{\psi}}{d t}=\operatorname{sign} \frac{d \psi}{d t}$. Furthermore, $\left.\bar{\psi}\right|_{a_{i}} ^{b_{i}}=\left.\psi\right|_{a_{i}} ^{b_{i}}$ for every index $i \in I$, and $\left\{t_{i}\right\}_{i \in I}$ is a set of basepoints for $(\varphi, \bar{\psi})$, i.e.

$$
\varphi^{*}\left(t_{i}\right)=\sigma_{i} \cdot \max _{\left[a_{i}, b_{i}\right]}\left\{\sigma_{i} \cdot \varphi^{*}\right\},
$$

where $\sigma_{i}$ denotes the sign taken by both $\frac{d \psi}{d t}$ and $\frac{d \bar{\psi}}{d t}$ on the open interval $J_{i}=\left(a_{i}, b_{i}\right)$. Therefore

$$
\begin{aligned}
\int_{a_{i}}^{b_{i}} & \varphi^{*}(t) \cdot \frac{d \bar{\psi}}{d t}(t) d t=\int_{a_{i}}^{b_{i}} \varphi^{*}(t) \cdot \sigma_{i} \cdot\left|\frac{d \bar{\psi}}{d t}(t)\right| d t \\
& \leq \int_{a_{i}}^{b_{i}} \max _{\left[a_{i}, b_{i}\right]}\left\{\varphi^{*} \cdot \sigma_{i}\right\} \cdot \frac{d \bar{\psi}}{d t}(t) \cdot \sigma_{i} d t \\
& =\int_{a_{i}}^{b_{i}} \varphi^{*}\left(t_{i}\right) \cdot \frac{d \bar{\psi}}{d t}(t) d t=\left.\varphi^{*}\left(t_{i}\right) \cdot \bar{\psi}\right|_{a_{i}} ^{b_{i}}=\left.\varphi^{*}\left(t_{i}\right) \cdot \psi\right|_{a_{i}} ^{b_{i}} .
\end{aligned}
$$

Hence,

$$
\int_{-\infty}^{+\infty} \varphi^{*}(t) \cdot \frac{d(\psi \circ h)}{d t}(t) d t \leq\left.\sum_{i \in I} \psi\right|_{a_{i}} ^{b_{i}} \cdot \varphi^{*}\left(t_{i}\right)=F(\varphi, \psi) .
$$


By substituting $\psi$ with $-\psi$ in the previous inequality (observe that $\mathcal{J}(-\psi)=\mathcal{J}(\psi)$ and $H_{-\psi}=H_{\psi}$ ), we get

$$
-\int_{-\infty}^{+\infty} \varphi^{*}(t) \cdot \frac{d(\psi \circ h)}{d t}(t) d t \leq \sum_{i \in I}-\left.\psi\right|_{a_{i}} ^{b_{i}} \cdot \varphi^{*}\left(\tilde{t}_{i}\right)=F(\varphi,-\psi)
$$

where $\left\{\tilde{t}_{i}\right\}_{i \in I}$ is a set of basepoints for $(\varphi,-\bar{\psi})$.

It follows that

$$
\left|\int_{-\infty}^{+\infty} \varphi^{*}(t) \cdot \frac{d(\psi \circ h)}{d t}(t) d t\right| \leq \max \{F(\varphi, \psi), F(\varphi,-\psi)\}
$$

for every $h \in H_{\psi}$. Therefore, the inequality

$$
\sup _{h \in H_{\psi}}\left|\int_{-\infty}^{+\infty} \varphi^{*}(t) \cdot \frac{d(\psi \circ h)}{d t}(t) d t\right| \leq \max \{F(\varphi, \psi), F(\varphi,-\psi)\}
$$

holds.

PART 2: $\sup _{h \in H_{\psi}}\left|\int_{-\infty}^{+\infty} \varphi^{*}(t) \cdot \frac{d(\psi \circ h)}{d t}(t) d t\right| \geq \max \{F(\varphi, \psi), F(\varphi,-\psi)\}$.

Assume $\bar{\psi}=\psi \circ h$ with $h \in H_{\psi}$. Once more, $\mathcal{J}(\bar{\psi})=\mathcal{J}(\psi), \operatorname{sign} \frac{d \bar{\psi}}{d s}=\operatorname{sign} \frac{d \psi}{d s}$ and $\left\{t_{i}\right\}_{i \in I}$ is a set of basepoints for $(\varphi, \bar{\psi})$, i.e.

$$
\varphi^{*}\left(t_{i}\right)=\sigma_{i} \cdot \max _{\left[a_{i}, b_{i}\right]}\left\{\sigma_{i} \cdot \varphi^{*}\right\}
$$

where $\sigma_{i}$ denotes the sign taken by $\frac{d \psi}{d s}$ and $\frac{d \bar{\psi}}{d s}$ on the interval $J_{i}$.

Let us choose an $\varepsilon>0$. In order to avoid the problem of some $t_{i}$ 's possibly belonging to the boundary of $J_{i}$, we define a new set $\left\{t_{i}^{\prime}\right\}_{i \in I}$ : for each interval $J_{i}=\left(a_{i}, b_{i}\right) \in \mathcal{J}(\bar{\psi})$, we choose a $t_{i}^{\prime} \in\left(a_{i}, b_{i}\right)$ such that $\left|\varphi^{*}\left(t_{i}\right)-\varphi^{*}\left(t_{i}^{\prime}\right)\right|<\frac{\varepsilon}{2^{i}}$.

For each positive integer $k \leq|I|$ let us choose a positive real number $\eta$ such that $\eta<\min \left\{\frac{t_{i}^{\prime}-a_{i}}{2}, \frac{b_{i}-t_{i}^{\prime}}{2}, \varepsilon\right\}$, for every $i \leq k-1$. Then we can consider an orientation-preserving diffeomorphism $h_{(\eta, k)} \in H_{\psi}$ such that

i): for every $i \leq k-1, h_{(\eta, k)}$ maps $\left(t_{i}^{\prime}-\frac{\eta}{2^{i}}, t_{i}^{\prime}+\frac{\eta}{2^{i}}\right)$ onto $\left(a_{i}+\frac{\eta}{2^{i}}, b_{i}-\frac{\eta}{2^{i}}\right)$;

ii): the restriction of $h_{(\eta, k)}$ to the set $\mathbb{R}-\bigcup_{i=0}^{k-1} J_{i}$ is the identity.

Recall also that, because $h_{(\eta, k)} \in H_{\psi}$, for every index $i$ the map $h_{(\eta, k)}$ takes the interval $J_{i}$ onto itself.

Since $\varphi$ is continuous, we can also assume to choose $\eta$ so small that the inequality $\left|\varphi^{*}\left(t_{i}^{\prime}\right)-\varphi^{*}\left(h_{(\eta, k)}^{-1}(t)\right)\right|<\frac{\varepsilon}{2^{i}}$ holds for any $t \in\left(a_{i}+\frac{\eta}{2^{i}}, b_{i}-\frac{\eta}{2^{i}}\right)$ and any index $i \leq k-1$.

Therefore, for any index $i \leq k-1$, by setting $t=h_{(\eta, k)}(s)$ and recalling that the sign of $\frac{d \psi}{d t}$ is constant in $\left(a_{i}, b_{i}\right)$ (we shall use this fact in several of the following 
passages),

$$
\begin{aligned}
& \left.|\psi|\right|_{a_{i}} ^{b_{i}} \cdot \varphi^{*}\left(t_{i}^{\prime}\right)-\int_{a_{i}}^{b_{i}} \varphi^{*}(s) \cdot \frac{d\left(\psi \circ h_{(\eta, k)}\right)}{d s}(s) d s \mid \\
& =\left.|\psi|\right|_{a_{i}} ^{b_{i}} \cdot \varphi^{*}\left(t_{i}^{\prime}\right)-\int_{a_{i}}^{b_{i}} \varphi^{*}(s) \cdot \frac{d \psi}{d s}\left(h_{(\eta, k)}(s)\right) \cdot \frac{d h_{(\eta, k)}}{d s}(s) d s \mid \\
& =\left.|\psi|\right|_{a_{i}} ^{b_{i}} \cdot \varphi^{*}\left(t_{i}^{\prime}\right)-\int_{a_{i}}^{b_{i}} \varphi^{*}\left(h_{(\eta, k)}^{-1}(t)\right) \cdot \frac{d \psi}{d t}(t) d t \mid \\
& \leq\left.|\psi|\right|_{a_{i}} ^{b_{i}} \cdot \varphi^{*}\left(t_{i}^{\prime}\right)-\int_{a_{i}+\frac{\eta}{2^{i}}}^{b_{i}-\frac{\eta}{2^{i}}} \varphi^{*}\left(h_{(\eta, k)}^{-1}(t)\right) \cdot \frac{d \psi}{d t}(t) d t \mid \\
& +\left|\int_{a_{i}}^{a_{i}+\frac{\eta}{2^{i}}} \varphi^{*}\left(h_{(\eta, k)}^{-1}(t)\right) \cdot \frac{d \psi}{d t}(t) d t\right|+\left|\int_{b_{i}-\frac{\eta}{2^{i}}}^{b_{i}} \varphi^{*}\left(h_{(\eta, k)}^{-1}(t)\right) \cdot \frac{d \psi}{d t}(t) d t\right| \\
& \leq\left.|\psi|\right|_{a_{i}} ^{b_{i}} \cdot \varphi^{*}\left(t_{i}^{\prime}\right)-\varphi^{*}\left(t_{i}^{\prime}\right) \cdot \int_{a_{i}+\frac{\eta}{2^{i}}}^{b_{i}-\frac{\eta}{2^{i}}} \frac{d \psi}{d t}(t) d t \mid \\
& +\left|\int_{a_{i}+\frac{\eta}{2^{i}}}^{b_{i}-\frac{\eta}{2^{i}}}\left(\varphi^{*}\left(t_{i}^{\prime}\right)-\varphi^{*}\left(h_{(\eta, k)}^{-1}(t)\right)\right) \cdot \frac{d \psi}{d t}(t) d t\right| \\
& +\quad \max |\varphi| \cdot \int_{a_{i}}^{a_{i}+\frac{\eta}{2^{2}}}\left|\frac{d \psi}{d t}(t)\right| d t+\max |\varphi| \cdot \int_{b_{i}-\frac{\eta}{2^{2}}}^{b_{i}}\left|\frac{d \psi}{d t}(t)\right| d t \\
& \leq\left|\left(\psi||_{a_{i}}^{b_{i}}-\left.\psi\right|_{a_{i}+\eta / 2^{i}} ^{b_{i}-\eta / 2^{i}}\right) \cdot \varphi^{*}\left(t_{i}^{\prime}\right)\right|+\int_{a_{i}+\frac{\eta}{2^{i}}}^{b_{i}-\frac{\eta}{2^{i}}}\left|\varphi^{*}\left(t_{i}^{\prime}\right)-\varphi^{*}\left(h_{(\eta, k)}^{-1}(t)\right)\right| \cdot\left|\frac{d \psi}{d t}(t)\right| d t \\
& +\left.|\psi|\right|_{a_{i}} ^{a_{i}+\eta / 2^{i}}+\left.\psi\right|_{b_{i}-\eta / 2^{i}} ^{b_{i}}|\cdot \max | \varphi \mid \\
& \leq\left.|\psi|\right|_{a_{i}} ^{a_{i}+\eta / 2^{i}}+\left.\psi\right|_{b_{i}-\eta / 2^{i}} ^{b_{i}}|\cdot \max | \varphi\left|+\frac{\varepsilon}{2^{i}} \cdot \int_{a_{i}+\frac{\eta}{2^{i}}}^{b_{i}-\frac{\eta}{2^{i}}}\right| \frac{d \psi}{d t}(t) \mid d t \\
& +\left.|\psi|\right|_{a_{i}} ^{a_{i}+\eta / 2^{i}}+\left.\psi\right|_{b_{i}-\eta / 2^{i}} ^{b_{i}}|\cdot \max | \varphi \mid \\
& =\left.|\psi|\right|_{a_{i}} ^{a_{i}+\eta / 2^{i}}+\left.\psi\right|_{b_{i}-\eta / 2^{i}} ^{b_{i}}|\cdot \max | \varphi\left|+\frac{\varepsilon}{2^{i}} \cdot\right| \psi||_{a_{i}+\eta / 2^{i}}^{b_{i}-\eta / 2^{i}} \mid \\
& +\left.|\psi|\right|_{a_{i}} ^{a_{i}+\eta / 2^{i}}+\left.\psi\right|_{b_{i}-\eta / 2^{i}} ^{b_{i}}|\cdot \max | \varphi \mid \\
& =2\left(\left.\left.|\psi|\right|_{a_{i}} ^{a_{i}+\eta / 2^{i}}|+| \psi\right|_{b_{i}-\eta / 2^{i}} ^{b_{i}} \mid\right) \cdot \max |\varphi|+\left.\frac{\varepsilon}{2^{i}} \cdot|\psi|\right|_{a_{i}+\eta / 2^{i}} ^{b_{i}-\eta / 2^{i}} \mid \\
& \leq 4 \cdot \frac{\eta}{2^{i}} \cdot \max \left|\frac{d \psi}{d t}\right| \cdot \max |\varphi|+\frac{\varepsilon}{2^{i}} \cdot(\max \psi-\min \psi) .
\end{aligned}
$$

It follows that, for every positive integer $k \leq|I|$ and every $\varepsilon>0$, a small enough positive $\eta=\eta(k, \varepsilon) \leq \varepsilon$ exists such that, denoting by $A_{k}$ the set $\bigcup_{i=0}^{k-1}\left(a_{i}, b_{i}\right)$, we 
have

$$
\begin{aligned}
& \left|\sum_{i=0}^{k-1}\left(\left.\psi\right|_{a_{i}} ^{b_{i}} \cdot \varphi^{*}\left(t_{i}\right)-\int_{A_{k}} \varphi^{*}(s) \cdot \frac{d\left(\psi \circ h_{(\eta, k)}\right)}{d s}(s) d s\right)\right| \\
\leq & \left|\sum_{i=0}^{k-1} \psi\right|_{a_{i}}^{b_{i}} \cdot\left(\varphi^{*}\left(t_{i}\right)-\varphi^{*}\left(t_{i}^{\prime}\right)\right) \mid \\
& +\left|\sum_{i=0}^{k-1}\left(\left.\psi\right|_{a_{i}} ^{b_{i}} \cdot \varphi^{*}\left(t_{i}^{\prime}\right)-\int_{A_{k}} \varphi^{*}(s) \cdot \frac{d\left(\psi \circ h_{(\eta, k)}\right)}{d s}(s) d s\right)\right| \\
\leq \quad & V_{\psi} \cdot \sum_{i=0}^{k-1} \frac{\varepsilon}{2^{i}}+\left.\sum_{i=0}^{k-1}|\psi|\right|_{a_{i}} ^{b_{i}} \cdot \varphi^{*}\left(t_{i}^{\prime}\right)-\int_{A_{k}} \varphi^{*}(s) \cdot \frac{d\left(\psi \circ h_{(\eta, k)}\right)}{d s}(s) d s \mid \\
\leq & 2 \varepsilon \cdot V_{\psi}+8 \eta \cdot \max \left|\frac{d \psi}{d t}\right| \cdot \max |\varphi|+2 \varepsilon \cdot(\max \psi-\min \psi) .
\end{aligned}
$$

Hence

$$
\begin{aligned}
& \left|\sum_{i=0}^{k-1} \psi\right|_{a_{i}}^{b_{i}} \cdot \varphi^{*}\left(t_{i}\right)-\int_{-\infty}^{+\infty} \varphi^{*}(s) \cdot \frac{d\left(\psi \circ h_{(\eta, k)}\right)}{d s}(s) d s \mid \\
& \leq 2 \varepsilon \cdot\left(V_{\psi}+\max \psi-\min \psi\right)+8 \eta \cdot \max \left|\frac{d \psi}{d t}\right| \cdot \max |\varphi| \\
& \quad+\left|\int_{\mathbb{R}-A_{k}} \varphi^{*}(s) \cdot \frac{d\left(\psi \circ h_{(\eta, k)}\right)}{d s}(s) d s\right| .
\end{aligned}
$$

By definition of $h_{(\eta, k)}$, the function $\psi \circ h_{(\eta, k)}$ equals $\psi$ on $\mathbb{R}-A_{k}$ and hence, when $I=\mathbb{N}$,

$$
\lim _{k \rightarrow|I|} \int_{\mathbb{R}-A_{k}} \varphi^{*}(s) \cdot \frac{d\left(\psi \circ h_{(\eta, k)}\right)}{d s}(s) d s=\lim _{k \rightarrow|I|} \int_{\mathbb{R}-A_{k}} \varphi^{*}(s) \cdot \frac{d \psi}{d s}(s) d s=0
$$

and analogously when $k=|I|$,

$$
\int_{\mathbb{R}-A_{k}} \varphi^{*}(s) \cdot \frac{d\left(\psi \circ h_{(\eta, k)}\right)}{d s}(s) d s=\int_{\mathbb{R}-A_{k}} \varphi^{*}(s) \cdot \frac{d \psi}{d s}(s) d s=0 .
$$

Therefore, recalling that $\eta \leq \varepsilon$, the following inequality holds for every large enough $k$ (if $|I|=\infty$ ), and for $k=|I|($ if $|I|<\infty)$ :

$$
\begin{aligned}
& \left|\sum_{i=0}^{k-1} \psi\right|_{a_{i}}^{b_{i}} \cdot \varphi^{*}\left(t_{i}\right)-\int_{-\infty}^{+\infty} \varphi^{*}(s) \cdot \frac{d\left(\psi \circ h_{(\eta, k)}\right)}{d s}(s) d s \mid \\
& \leq 2 \varepsilon \cdot\left(V_{\psi}+\max \psi-\min \psi+4 \cdot \max \left|\frac{d \psi}{d t}\right| \cdot \max |\varphi|\right)+2 \varepsilon
\end{aligned}
$$


Hence

$$
\begin{aligned}
& \left|\sum_{i=0}^{|I|-1} \psi\right|_{a_{i}}^{b_{i}} \cdot \varphi^{*}\left(t_{i}\right)-\int_{-\infty}^{+\infty} \varphi^{*}(s) \cdot \frac{d\left(\psi \circ h_{(\eta, k)}\right)}{d s}(s) d s \mid \\
& \leq 2 \varepsilon \cdot\left(V_{\psi}+\max \psi-\min \psi+4 \cdot \max \left|\frac{d \psi}{d t}\right| \cdot \max |\varphi|+1\right) \\
& \quad+\left|\sum_{i=k}^{|I|-1} \psi\right|_{a_{i}}^{b_{i}} \cdot \varphi^{*}\left(t_{i}\right) \mid
\end{aligned}
$$

(here and in the sequel, when $I=\mathbb{N}$, we set $|I|-1=\infty$ ).

Since $F(\varphi, \psi)=\left.\sum_{i=0}^{|I|-1} \psi\right|_{a_{i}} ^{b_{i}} \cdot \varphi^{*}\left(t_{i}\right)$ and it is finite, if $k$ is large enough (in case $|I|=\infty$ ), or $k=|I|$ (in case $|I|<\infty$ ) we get $\left|\sum_{i=k}^{|I|-1} \psi\right|_{a_{i}}^{b_{i}} \cdot \varphi^{*}\left(t_{i}\right) \mid \leq 2 \varepsilon$. Hence

$$
\begin{gathered}
\left|F(\varphi, \psi)-\int_{-\infty}^{+\infty} \varphi^{*}(s) \cdot \frac{d\left(\psi \circ h_{(\eta, k)}\right)}{d s}(s) d s\right| \\
\leq 2 \varepsilon \cdot\left(V_{\psi}+\max \psi-\min \psi+4 \cdot \max \left|\frac{d \psi}{d t}\right| \cdot \max |\varphi|+2\right) .
\end{gathered}
$$

Inequality (9) proves that for every $\bar{\varepsilon}>0$, an orientation-preserving diffeomorphism $h^{+} \in H_{\psi}$ exists such that

$$
\left|\int_{-\infty}^{+\infty} \varphi^{*}(t) \cdot \frac{d\left(\psi \circ h^{+}\right)}{d t}(t) d t\right| \geq \int_{-\infty}^{+\infty} \varphi^{*}(t) \cdot \frac{d\left(\psi \circ h^{+}\right)}{d t}(t) d t \geq F(\varphi, \psi)-\bar{\varepsilon}
$$

By replacing $\psi$ with $-\psi$ and observing that $H_{-\psi}=H_{\psi}$ we get, for every $\bar{\varepsilon}>0$, an orientation-preserving diffeomorphism $h^{-} \in H_{\psi}$ such that

$$
\left|\int_{-\infty}^{+\infty} \varphi^{*}(t) \cdot \frac{d\left(\psi \circ h^{-}\right)}{d t}(t) d t\right| \geq-\int_{-\infty}^{+\infty} \varphi^{*}(t) \cdot \frac{d\left(\psi \circ h^{-}\right)}{d t}(t) d t \geq F(\varphi,-\psi)-\bar{\varepsilon} .
$$

Therefore the inequality

$$
\sup _{h \in H_{\psi}}\left|\int_{-\infty}^{+\infty} \varphi^{*}(t) \cdot \frac{d(\psi \circ h)}{d t}(t) d t\right| \geq \max \{F(\varphi, \psi), F(\varphi,-\psi)\}
$$

holds. This implies our claim.

The next result, proved by applying the previous lemma, motivates the introduction of the functional $F$.

Proposition 4.5. $\|\varphi\|_{[\psi]}=\sup _{\hat{\psi} \in[\psi]} \max \{F(\varphi, \hat{\psi}), F(\varphi,-\hat{\psi})\}$.

Proof. Lemma 4.4 implies that

$$
\begin{aligned}
& \|\varphi\|_{[\psi]}=\sup _{\hat{\psi} \in[\psi]}\left|\int_{-\infty}^{+\infty} \varphi^{*}(t) \cdot \frac{d \hat{\psi}}{d t}(t) d t\right| \\
= & \sup _{\hat{\psi} \in[\psi]} \sup _{h \in H_{\hat{\psi}}}\left|\int_{-\infty}^{+\infty} \varphi^{*}(t) \cdot \frac{d(\hat{\psi} \circ h)}{d t}(t) d t\right|=\sup _{\hat{\psi} \in[\psi]} \max \{F(\varphi, \hat{\psi}), F(\varphi,-\hat{\psi})\} .
\end{aligned}
$$


4.3. Standard RPI-norms as absolute values of linear combinations of Dirac deltas. The next theorem simplifies the computation of the standard RPInorms, bypassing the concept of basepoints for the pair $(\varphi, \psi)$. First we define a new set $\mathcal{T}(\varphi, \psi)$ based on the natural ordering $\preceq$, previously introduced on the set $\mathcal{J}(\psi)$. We recall that $J_{i} \preceq J_{j}$ if and only if $a_{i} \leq a_{j}$. This order does not need to coincide with the order induced by the index $i$. We also recall that the set $I$ indexing $\mathcal{J}(\psi)$ can be assumed to be either the finite set $\{0,1 \ldots, n-1\}$ or the set $\mathbb{N}$.

Definition 4.6. Let $\varphi, \psi \in A S^{1}(\mathbb{R})-\{\mathbf{0}\}$, and let $[a, b]$ be the smallest closed interval containing the support of $\frac{d \varphi^{*}}{d t}$. We shall denote by $\mathcal{T}(\varphi, \psi)$ the set of all the sequences $\left(\tau_{i}\right)$ of points of $[a, b]$ such that $J_{i_{1}} \preceq J_{i_{2}}$ implies $\tau_{i_{1}} \leq \tau_{i_{2}}$, for every $i_{1}, i_{2} \in I$, and $\tau_{i}=b$ if $i \notin I$. The sequences in $\mathcal{T}(\varphi, \psi)$ will be said to be compatible with $(\varphi, \psi)$. The terms $\tau_{i}=b$ with $i \notin I$ will be called dummy terms of the sequence $\left(\tau_{i}\right) \in \mathcal{T}(\varphi, \psi)$.

Remark 4.7. When $I=\mathbb{N}$, if a set of basepoints $\left\{t_{i}\right\}_{i \in I}$ is given, then the sequence $\left(t_{i}\right)$ is a compatible sequence itself. When $I$ is finite, starting from a set of basepoints, we can obtain a compatible sequence by adding infinitely many dummy terms $\tau_{i}=b$ to our finite sequence. As a matter of fact, the dummy terms will not be used in our computations.

Remark 4.8. For every $\left(\tau_{i}\right) \in \mathcal{T}(\varphi, \psi)$, the series $\left.\sum_{i \in I} \psi\right|_{a_{i}} ^{b_{i}} \cdot \varphi^{*}\left(\tau_{i}\right)$ converges, since $\left|\sum_{i \in I} \psi\right|_{a_{i}}^{b_{i}} \cdot \varphi^{*}\left(\tau_{i}\right)|\leq \max | \varphi \mid \cdot V_{\psi}$.

Theorem 4.9. If $\varphi, \psi \neq \mathbf{0}$, then $\|\varphi\|_{[\psi]}=\sup _{\left(\tau_{i}\right) \in \mathcal{T}(\varphi, \psi)}\left|\sum_{i \in I} \psi\right|_{a_{i}}^{b_{i}} \cdot \varphi^{*}\left(\tau_{i}\right) \mid$.

Proof. If $\hat{\psi} \in[\psi]$, an orientation-preserving diffeomorphism $h \in D_{+}^{1}(\mathbb{R})$ exists such that $\psi=\hat{\psi} \circ h$, and $\mathcal{J}(\hat{\psi})=\left\{h\left(J_{i}\right)\right\}_{i \in I}$. For each index $i$, we define the open interval $\left(\alpha_{i}, \beta_{i}\right)$ by setting $\left(\alpha_{i}, \beta_{i}\right)=h\left(J_{i}\right)$. Let us choose a set $\left\{\hat{t}_{i}^{+}\right\}$of basepoints for the pair $(\varphi, \hat{\psi})$ by taking each $\hat{t}_{i}^{+}$in the closure of the interval $h\left(J_{i}\right)$. Analogously, let us choose a set $\left\{\hat{t}_{i}^{-}\right\}$of basepoints for the pair $(\varphi,-\hat{\psi})$ by taking each $\hat{t}_{i}^{-}$in the closure of the interval $h\left(J_{i}\right)$.

Because of the definition of $F$, we obtain that

$$
\begin{gathered}
F(\varphi, \hat{\psi})=\left.\sum_{i \in I} \hat{\psi}\right|_{\alpha_{i}} ^{\beta_{i}} \cdot \varphi^{*}\left(\hat{t}_{i}^{+}\right)=\left.\sum_{i \in I} \psi\right|_{a_{i}} ^{b_{i}} \cdot \varphi^{*}\left(\hat{t}_{i}^{+}\right), \\
F(\varphi,-\hat{\psi})=-\left.\sum_{i \in I} \hat{\psi}\right|_{\alpha_{i}} ^{\beta_{i}} \cdot \varphi^{*}\left(\hat{t}_{i}^{-}\right)=-\left.\sum_{i \in I} \psi\right|_{a_{i}} ^{b_{i}} \cdot \varphi^{*}\left(\hat{t}_{i}^{-}\right) .
\end{gathered}
$$

Therefore,

$$
\max \{F(\varphi, \hat{\psi}), F(\varphi,-\hat{\psi})\} \leq \max \left\{\left.\left.\left|\sum_{i \in I} \psi\right|\right|_{a_{i}} ^{b_{i}} \cdot \varphi^{*}\left(\hat{t}_{i}^{+}\right)|,| \sum_{i \in I} \psi\right|_{a_{i}} ^{b_{i}} \cdot \varphi^{*}\left(\hat{t}_{i}^{-}\right) \mid\right\} .
$$

Let $\left(\hat{t}_{i}^{+}\right)$be a compatible sequence for $(\varphi, \hat{\psi})$ obtained from the set of basepoints $\left\{\hat{t}_{i}^{+}\right\}$, and analogously, let $\left(\hat{t}_{i}^{-}\right)$be a compatible sequence for $(\varphi,-\hat{\psi})$ obtained from the set of basepoints $\left\{\hat{t}_{i}^{-}\right\}$(recall Remark 4.7). It is easy to see that $\left(\hat{t}_{i}^{+}\right)$and $\left(\hat{t}_{i}^{-}\right)$ are compatible sequences also for $(\varphi, \psi)$. Therefore, for any $\hat{\psi} \in[\psi]$, the inequality

$$
\max \{F(\varphi, \hat{\psi}), F(\varphi,-\hat{\psi})\} \leq \sup _{\left(\tau_{i}\right) \in \mathcal{T}(\varphi, \psi)}\left|\sum_{i \in I} \psi\right|_{a_{i}}^{b_{i}} \cdot \varphi^{*}\left(\tau_{i}\right) \mid
$$


holds. From Prop. 4.5 the inequality

$$
\|\varphi\|_{[\psi]} \leq \sup _{\left(\tau_{i}\right) \in \mathcal{T}(\varphi, \psi)}\left|\sum_{i \in I} \psi\right|_{a_{i}}^{b_{i}} \cdot \varphi^{*}\left(\tau_{i}\right) \mid
$$

follows.

On the other hand, because of the continuity of $\varphi$, for every $\left(\tau_{i}\right) \in \mathcal{T}(\varphi, \psi)$, every positive integer $k \leq|I|$ and every $\varepsilon>0$, an orientation-preserving diffeomorphism $h_{k, \varepsilon} \in D_{+}^{1}(\mathbb{R})$ exists, such that the distance between the number $\varphi^{*}\left(\tau_{i}\right)$ and each value in the set $\varphi^{*}\left(h_{k, \varepsilon}\left(J_{i}\right)\right)$ is not greater than $\varepsilon$, for $i \leq k-1$ (it is sufficient to choose a diffeomorphism taking each $J_{i}$ into an interval contained in a small neighborhood of $\left.\tau_{i}\right)$. We point out that here we are using the hypothesis that $\left(\tau_{i}\right)$ is a sequence compatible with $(\varphi, \psi)$.

Let us consider $\hat{\psi}_{k, \varepsilon}=\psi \circ h_{k, \varepsilon}^{-1}$, and choose a set $\left\{\hat{t}_{i}^{+}\right\}$of basepoints for the pair $\left(\varphi, \hat{\psi}_{k, \varepsilon}\right)$ and a set $\left\{\hat{t}_{i}^{-}\right\}$of basepoints for the pair $\left(\varphi,-\hat{\psi}_{k, \varepsilon}\right)$. For each index $i \leq k-1$, we define the open interval $\left(\alpha_{i}^{\prime}, \beta_{i}^{\prime}\right)$ by setting $\left(\alpha_{i}^{\prime}, \beta_{i}^{\prime}\right)=h_{k, \varepsilon}\left(J_{i}\right)$. As before, we observe that $\mathcal{J}\left(\hat{\psi}_{k, \varepsilon}\right)=\left\{h_{k, \varepsilon}\left(J_{i}\right)\right\}_{i \in I}$ and that $\hat{t}_{i}^{+}, \hat{t}_{i}^{-}$belong to the closure of $h_{k, \varepsilon}\left(J_{i}\right)$ for every index $i \leq k-1$. Since $\left|\varphi^{*}\left(\hat{t}_{i}^{+}\right)-\varphi^{*}\left(\tau_{i}\right)\right| \leq \varepsilon$ and $\left|\varphi^{*}\left(\hat{t}_{i}^{-}\right)-\varphi^{*}\left(\tau_{i}\right)\right| \leq \varepsilon$, from $\left.\hat{\psi}_{k, \varepsilon}\right|_{\alpha_{i}^{\prime}} ^{\beta_{i}^{\prime}}=\left.\psi\right|_{a_{i}} ^{b_{i}}$ we get

$$
\left|\sum_{i=0}^{k-1} \hat{\psi}_{k, \varepsilon}\right|_{\alpha_{i}^{\prime}}^{\beta_{i}^{\prime}} \cdot \varphi^{*}\left(\hat{t}_{i}^{+}\right)-\left.\sum_{i=0}^{k-1} \psi\right|_{a_{i}} ^{b_{i}} \cdot \varphi^{*}\left(\tau_{i}\right) \mid \leq \varepsilon \cdot V_{\psi}
$$

and

$$
\left|\sum_{i=0}^{k-1} \hat{\psi}_{k, \varepsilon}\right|_{\alpha_{i}^{\prime}}^{\beta_{i}^{\prime}} \cdot \varphi^{*}\left(\hat{t}_{i}^{-}\right)-\left.\sum_{i=0}^{k-1} \psi\right|_{a_{i}} ^{b_{i}} \cdot \varphi^{*}\left(\tau_{i}\right) \mid \leq \varepsilon \cdot V_{\psi} .
$$

Furthermore,

$$
\left.\left|\sum_{i=k}^{|I|-1} \hat{\psi}_{k, \varepsilon}\right|_{\alpha_{i}^{\prime}}^{\beta_{i}^{\prime}} \cdot \varphi^{*}\left(\hat{t}_{i}^{+}\right)|\leq \max | \varphi\left|\cdot \sum_{i=k}^{|I|-1}\right| \hat{\psi}_{k, \varepsilon}\right|_{\alpha_{i}^{\prime}} ^{\beta_{i}^{\prime}}|=\max | \varphi\left|\cdot \sum_{i=k}^{|I|-1}\right| \psi||_{a_{i}}^{b_{i}} \mid .
$$

Since $\psi$ is a function of bounded variation, if $k$ is large enough we get

$$
\left|\sum_{i=k}^{|I|-1} \hat{\psi}_{k, \varepsilon}\right|_{\alpha_{i}^{\prime}}^{\beta_{i}^{\prime}} \cdot \varphi^{*}\left(\hat{t}_{i}^{+}\right) \mid \leq \varepsilon
$$

(Here and in the following, $k$ large enough means $k=|I|$ if $|I|$ is finite, and in this case every empty summation is assumed to take the value 0. .)

Analogously, if $k$ is large enough we get

$$
\left.\left|\sum_{i=k}^{|I|-1} \hat{\psi}_{k, \varepsilon}\right|_{\alpha_{i}^{\prime}}^{\beta_{i}^{\prime}} \cdot \varphi^{*}\left(\hat{t}_{i}^{-}\right)|\leq \varepsilon, \quad| \sum_{i=k}^{|I|-1} \psi\right|_{a_{i}} ^{b_{i}} \cdot \varphi^{*}\left(\tau_{i}\right) \mid \leq \varepsilon .
$$

Therefore for every $\varepsilon>0$ we can find a large enough index $k$ such that

$$
\left|\sum_{i \in I} \hat{\psi}_{k, \varepsilon}\right|_{\alpha_{i}^{\prime}}^{\beta_{i}^{\prime}} \cdot \varphi^{*}\left(\hat{t}_{i}^{+}\right)-\left.\sum_{i \in I} \psi\right|_{a_{i}} ^{b_{i}} \cdot \varphi^{*}\left(\tau_{i}\right) \mid \leq \varepsilon \cdot V_{\psi}+2 \varepsilon
$$


and

$$
\left|\sum_{i \in I} \hat{\psi}_{k, \varepsilon}\right|_{\alpha_{i}^{\prime}}^{\beta_{i}^{\prime}} \cdot \varphi^{*}\left(\hat{t}_{i}^{-}\right)-\left.\sum_{i \in I} \psi\right|_{a_{i}} ^{b_{i}} \cdot \varphi^{*}\left(\tau_{i}\right) \mid \leq \varepsilon \cdot V_{\psi}+2 \varepsilon .
$$

By recalling the definition of $F$ we obtain

$$
\begin{gathered}
F\left(\varphi, \hat{\psi}_{k, \varepsilon}\right)+\varepsilon \cdot V_{\psi}+2 \varepsilon \geq\left.\sum_{i \in I} \psi\right|_{a_{i}} ^{b_{i}} \cdot \varphi^{*}\left(\tau_{i}\right), \\
F\left(\varphi,-\hat{\psi}_{k, \varepsilon}\right)+\varepsilon \cdot V_{\psi}+2 \varepsilon \geq-\left.\sum_{i \in I} \psi\right|_{a_{i}} ^{b_{i}} \cdot \varphi^{*}\left(\tau_{i}\right) .
\end{gathered}
$$

These last two inequalities and the arbitrariness of $\varepsilon$ imply that

$$
\sup _{\hat{\psi} \in[\psi]} \max \{F(\varphi, \hat{\psi}), F(\varphi,-\hat{\psi})\} \geq\left|\sum_{i \in I} \psi\right|_{a_{i}}^{b_{i}} \cdot \varphi^{*}\left(\tau_{i}\right) \mid
$$

for any $\left(\tau_{i}\right) \in \mathcal{T}(\varphi, \psi)$. It follows that

$$
\sup _{\hat{\psi} \in[\psi]} \max \{F(\varphi, \hat{\psi}), F(\varphi,-\hat{\psi})\} \geq \sup _{\left(\tau_{i}\right) \in \mathcal{T}(\varphi, \psi)}\left|\sum_{i \in I} \psi\right|_{a_{i}}^{b_{i}} \cdot \varphi^{*}\left(\tau_{i}\right) \mid .
$$

From Prop. 4.5 the inequality

$$
\|\varphi\|_{[\psi]} \geq \sup _{\left(\tau_{i}\right) \in \mathcal{T}(\varphi, \psi)}\left|\sum_{i \in I} \psi\right|_{a_{i}}^{b_{i}} \cdot \varphi^{*}\left(\tau_{i}\right) \mid
$$

follows. Hence our statement is proved.

4.4. Optimal sequences in $\mathcal{T}(\varphi, \psi)$. The previous Theorem 4.9 raises an interesting issue: is the sup equaling $\|\varphi\|_{[\psi]}$ actually a max? In Prop. 4.12 we shall give an affirmative answer to this question.

We consider the following definition:

Definition 4.10. Every sequence $\left(\bar{\tau}_{i}\right) \in \mathcal{T}(\varphi, \psi)$ such that $\|\varphi\|_{[\psi]}=$ $\left.\left|\sum_{i \in I} \psi\right|\right|_{a_{i}} ^{b_{i}} \cdot \varphi^{*}\left(\bar{\tau}_{i}\right) \mid$ is said to be optimal for $(\varphi, \psi)$. The set of all optimal sequences for $(\varphi, \psi)$ will be denoted by $\mathcal{O}(\varphi, \psi)$.

In the sequel, optimal sequences will be obtained as the limit of convergent subsequences of sequences in $\mathcal{T}(\varphi, \psi)$. Therefore we shall need the following lemma.

Lemma 4.11. From each sequence $\left(T^{n}\right)$ of sequences that belong to $\mathcal{T}(\varphi, \psi)$ it is possible to extract a subsequence that converges pointwise to a sequence $\bar{T} \in \mathcal{T}(\varphi, \psi)$.

Proof. Consider the smallest interval $[a, b]$ containing the compact support of $\frac{d \varphi^{*}}{d t}$. Let $\left(T^{n}\right)$ be a sequence of sequences belonging to $\mathcal{T}(\varphi, \psi)$. For every fixed $n$, $T^{n}=\left(t_{i}^{n}\right)$ with $t_{i}^{n} \in[a, b]$. Hence, the sequence $\left(T^{n}\right)$ admits a subsequence $\left(T^{n_{r}^{0}}\right)$ (varying $r$ ) such that $\left(t_{0}^{n_{r}^{0}}\right)$ converges to some $\bar{\tau}_{0} \in[a, b]$. The sequence $\left(T^{n_{r}^{0}}\right)$ admits a subsequence $\left(T^{n_{r}^{1}}\right)$ such that $\left(t_{1}^{n_{r}^{1}}\right)$ converges to some $\bar{\tau}_{1} \in[a, b]$. Since $\left(T^{n_{r}^{1}}\right)$ is a subsequence of $\left(T^{n_{r}^{0}}\right)$, it still holds that $\left(t_{0}^{n_{r}^{1}}\right)$ converges to $\bar{\tau}_{0}$. By iterating this argument, for every $s \in \mathbb{N}$, we can extract a subsequence $\left(T^{n_{r}^{s+1}}\right)$ from $\left(T_{r}^{n_{r}^{s}}\right)$ such that the sequence $\left(t_{i}^{n_{r}^{s+1}}\right)$ converges to $\bar{\tau}_{i} \in[a, b]$ (varying $r$ ) for 
every fixed $i$ with $0 \leq i \leq s+1$. By construction, the diagonal sequence $\left(T^{n_{r}^{r}}\right)$ is a subsequence of $\left(T^{n}\right)$. Furthermore, for every $s \in \mathbb{N},\left(T^{n_{r}^{r}}\right)$ is a subsequence of $\left(T^{n_{r}^{s}}\right)$ if we ignore the first $s$ terms of both these sequences. Hence $\lim _{r \rightarrow+\infty} t_{i}^{n_{r}^{r}}=\bar{\tau}_{i}$ for every fixed $i \in \mathbb{N}$. Finally, $\left(\bar{\tau}_{i}\right) \in \mathcal{T}(\varphi, \psi)$ since each $\bar{\tau}_{i} \in[a, b]$, and for every $i_{1}, i_{2} \in I$, if $J_{i_{1}} \preceq J_{i_{2}}$, then $t_{i_{1}}^{n_{r}^{r}} \leq t_{i_{2}}^{n_{r}^{r}}$ for every $r$, implying that $\lim _{r \rightarrow+\infty} t_{i_{1}}^{n_{r}^{r}} \leq$ $\lim _{r \rightarrow+\infty} t_{i_{2}}^{n_{r}^{r}}$. Hence the sequence $\left(T^{n_{r}^{r}}\right)$ proves our statement.

In the following pages, each sequence obtained by the method described in the proof of the previous lemma will be said to be "obtained by a diagonalization process".

Proposition 4.12. If $\varphi, \psi \neq \mathbf{0}$, then $\mathcal{O}(\varphi, \psi)$ is not empty.

Proof. On the basis of Theorem 4.9, for each $n \in \mathbb{N}$ we can take a sequence $T^{n}=$ $\left(t_{i}^{n}\right) \in \mathcal{T}(\varphi, \psi)$ in such a way that $\|\varphi\|_{[\psi]}=\lim _{n \rightarrow \infty}\left|\sum_{i \in I} \psi\right|_{a_{i}}^{b_{i}} \cdot \varphi^{*}\left(t_{i}^{n}\right) \mid$. By Lemma 4.11, the sequence of sequences $\left(T^{n}\right)$ admits a subsequence $\left(T^{n_{r}}\right)$ that pointwise converges to a sequence $\bar{T}=\left(\bar{\tau}_{i}\right)$ compatible with $(\varphi, \psi)$. If we denote each sequence $T^{n_{r}}$ by $\left(t_{i}^{n_{r}}\right)$ (varying $i$ ), the following equalities hold:

$$
\left.\left.\left|\sum_{i \in I} \psi\right|_{a_{i}}^{b_{i}} \cdot \varphi^{*}\left(\bar{\tau}_{i}\right)|=| \sum_{i \in I} \psi\right|_{a_{i}} ^{b_{i}} \cdot \lim _{r \rightarrow \infty} \varphi^{*}\left(t_{i}^{n_{r}}\right)\left|=\lim _{r \rightarrow \infty}\right| \sum_{i \in I} \psi\right|_{a_{i}} ^{b_{i}} \cdot \varphi^{*}\left(t_{i}^{n_{r}}\right) \mid=\|\varphi\|_{[\psi]},
$$

where the second equality follows from the fact that $\psi$ has bounded variation.

However, a stronger result holds, stating that optimal sequences for $(\varphi, \psi)$ exist, containing only critical points for $\varphi^{*}$. For its proof, we need a preliminary lemma.

Lemma 4.13. From each sequence $\left(T^{n}\right)$ of sequences in $\mathcal{O}(\varphi, \psi)$ it is possible to extract a subsequence that converges pointwise to a sequence $\bar{T} \in \mathcal{O}(\varphi, \psi)$.

Proof. By Lemma 4.11, $\left(T^{n}\right)$ admits a subsequence $\left(T^{n_{r}}\right)$ that converges pointwise to a sequence $\bar{T} \in \mathcal{T}(\varphi, \psi)$. By recalling that $\psi$ has bounded variation, it is easy to verify that $\bar{T} \in \mathcal{O}(\varphi, \psi)$ (cf. the equalities (10) in the previous proof of Proposition 4.12).

Now we can prove the following result, improving Proposition 4.12

Proposition 4.14. If $\varphi, \psi \neq \mathbf{0}$, then a sequence $\left(\hat{\tau}_{i}\right) \in \mathcal{O}(\varphi, \psi)$ exists, where each $\hat{\tau}_{i}$ is a critical point of $\varphi^{*}$.

Proof. Proposition 4.12 shows that the set $\mathcal{O}(\varphi, \psi)$ of all optimal sequences in $\mathcal{T}(\varphi, \psi)$ is not empty. Let $K_{\varphi^{*}}$ be the set of all critical points of $\varphi^{*}$. If $T=\left(\tau_{i}\right) \in$ $\mathcal{O}(\varphi, \psi)$, we define the weight $w(T)=\sum_{i \in I} \gamma_{i} \cdot|\psi|_{a_{i}}^{b_{i}} \mid$, where $\gamma_{i}=\min \left\{\tau_{i}-x \mid x \in\right.$ $K_{\varphi^{*}}, x \leq \tau_{i}$ \} (in other words, $\gamma_{i}$ is the distance between $\tau_{i}$ and the first critical point of $\varphi^{*}$ on its left). This positive term series converges, since it is smaller than $\left.(b-a) \cdot \sum_{i \in I}|\psi|\right|_{a_{i}} ^{b_{i}} \mid \leq(b-a) \cdot V_{\psi}$.

For any $n \in \mathbb{N}$ we can take a sequence $T^{n}=\left(\tau_{i}^{n}\right) \in \mathcal{O}(\varphi, \psi)$ in such a way that $\lim _{n \rightarrow \infty} w\left(T^{n}\right)=\inf _{T \in \mathcal{O}(\varphi, \psi)} w(T)$. By Lemma 4.13, we can extract from $\left(T^{n}\right)$ a subsequence converging pointwise to an optimal sequence $\widehat{T}=\left(\hat{\tau}_{i}\right)$.

Let us set $\gamma_{i}^{n}=\min \left\{\tau_{i}^{n}-x \mid x \in K_{\varphi^{*}}, x \leq \tau_{i}^{n}\right\}$ for every $n, i \in \mathbb{N}$, and $\widehat{\gamma}_{i}=$ $\min \left\{\hat{\tau}_{i}-x \mid x \in K_{\varphi^{*}}, x \leq \hat{\tau}_{i}\right\}$ for every $i \in \mathbb{N}$. Since the set $K_{\varphi^{*}}$ is closed, we can easily prove that, for every $i \in \mathbb{N}$, either $\widehat{\gamma}_{i}=\lim _{n \rightarrow \infty} \gamma_{i}^{n}$ or $\widehat{\gamma}_{i}=0$, although 
$\lim _{n \rightarrow \infty} \gamma_{i}^{n} \neq 0$. By recalling once again that $\psi$ has bounded variation, it follows that $\lim _{n \rightarrow \infty} w\left(T^{n}\right) \geq w(\widehat{T})$. Since $\lim _{n \rightarrow \infty} w\left(T^{n}\right)=\inf _{T \in \mathcal{O}(\varphi, \psi)} w(T)$, we get $w(\widehat{T})=\inf _{T \in \mathcal{O}(\varphi, \psi)} w(T)$.

Now we prove by contradiction that $w(\widehat{T})=0$. Assume $w(\widehat{T})>0$. Then an index $j \in I$ exists such that $\hat{\tau}_{j} \notin K_{\varphi^{*}}$ and, since $K_{\varphi^{*}}$ is closed, we can find an $\eta>0$ for which the closure of the open interval $U=\left(\hat{\tau}_{j}-\eta, \hat{\tau}_{j}+\eta\right)$ does not meet $K_{\varphi^{*}}$. We want to show that we can move all points of $\widehat{T}$ in $U$ leftwards, and get an optimal sequence with a weight that is strictly less than $w(\widehat{T})$. This will generate our contradiction.

In order to do that, let us consider a $C^{1}$-function $\rho: \mathbb{R} \rightarrow \mathbb{R}$ such that

- $\rho=0$ outside $U$;

- $\rho>0$ in $U$;

- $\max _{\bar{U}}\left|\frac{d \rho}{d t}\right|<\min _{\bar{U}}\left|\frac{d \varphi^{*}}{d t}\right|$.

The last hypothesis guarantees that the function $\varphi^{+}=\varphi^{*}+\rho$ is a (possibly orientation-reversing) diffeomorphism from $U$ onto its image $\varphi^{+}(U)$. Since $\varphi^{+}(U)=\varphi^{*}(U)$, we can consider the function from $U$ to $U$ that takes each point $t$ to the unique point $t^{\prime}$ such that $\varphi^{*}\left(t^{\prime}\right)=\varphi^{+}(t)$ (observe that either both $\varphi^{+}$and $\varphi^{*}$ are strictly increasing in $U$ or both $\varphi^{+}$and $\varphi^{*}$ are strictly decreasing in $U$ ). We can extend this function to a function $h^{+}: \mathbb{R} \rightarrow \mathbb{R}$ by defining it as equal to the identity outside $U$. We can immediately verify that $h^{+}$is an orientation-preserving diffeomorphism, since $\frac{d \varphi^{*}}{d t}$ and $\frac{d \varphi^{+}}{d t}$ take the same sign in $U$. Analogously, the function $\varphi^{-}=\varphi^{*}-\rho$ is a diffeomorphism from $U$ onto its image $\varphi^{-}(U)=\varphi^{*}(U)$. Hence we can consider the function from $U$ to $U$ that takes each point $t$ to the unique point $t^{\prime}$ such that $\varphi^{*}\left(t^{\prime}\right)=\varphi^{-}(t)$. We can extend this function to a function $h^{-}: \mathbb{R} \rightarrow \mathbb{R}$ by defining it as equal to the identity outside $U$, and $h^{-}$is an orientation-preserving diffeomorphism.

Now, let us define two new sequences $\left(\tau_{i}^{+}\right)$and $\left(\tau_{i}^{-}\right)$. For every $i \in \mathbb{N}$ we set $\tau_{i}^{+}=h^{+}\left(\hat{\tau}_{i}\right), \tau_{i}^{-}=h^{-}\left(\hat{\tau}_{i}\right)$, so that $\varphi^{*}\left(\tau_{i}^{+}\right)=\varphi^{+}\left(\hat{\tau}_{i}\right)$ and $\varphi^{*}\left(\tau_{i}^{-}\right)=\varphi^{-}\left(\hat{\tau}_{i}\right)$.

Since $h^{+}$is an orientation-preserving diffeomorphism, $\hat{\tau}_{i} \leq \hat{\tau}_{j}$ if and only if $\tau_{i}^{+} \leq$ $\tau_{j}^{+}$. That means that also $\left(\tau_{i}^{+}\right)$is a sequence compatible with $(\varphi, \psi)$. Analogously, also $\left(\tau_{i}^{-}\right)$is a sequence compatible with $(\varphi, \psi)$. Since the sequence $\left(\hat{\tau}_{i}\right)$ is optimal, if $\left.\sum_{i \in I} \psi\right|_{a_{i}} ^{b_{i}} \cdot \varphi^{*}\left(\hat{\tau}_{i}\right) \geq 0$, the following statements hold:

$$
\begin{gathered}
\left.\sum_{i \in I} \psi\right|_{a_{i}} ^{b_{i}} \cdot \varphi^{*}\left(\hat{\tau}_{i}\right)-\left.\sum_{i \in I} \psi\right|_{a_{i}} ^{b_{i}} \cdot \varphi^{*}\left(\tau_{i}^{+}\right)=-\left.\sum_{i \in I} \psi\right|_{a_{i}} ^{b_{i}} \cdot \rho\left(\hat{\tau}_{i}\right) \geq 0, \\
\left.\sum_{i \in I} \psi\right|_{a_{i}} ^{b_{i}} \cdot \varphi^{*}\left(\hat{\tau}_{i}\right)-\left.\sum_{i \in I} \psi\right|_{a_{i}} ^{b_{i}} \cdot \varphi^{*}\left(\tau_{i}^{-}\right)=\left.\sum_{i \in I} \psi\right|_{a_{i}} ^{b_{i}} \cdot \rho\left(\hat{\tau}_{i}\right) \geq 0
\end{gathered}
$$

and hence $\left.\sum_{i \in I} \psi\right|_{a_{i}} ^{b_{i}} \cdot \rho\left(\hat{\tau}_{i}\right)=0$.

On the other hand, if $\left.\sum_{i \in I} \psi\right|_{a_{i}} ^{b_{i}} \cdot \varphi^{*}\left(\hat{\tau}_{i}\right)<0$, the optimality of $\left(\hat{\tau}_{i}\right)$ implies the following statements:

$$
\begin{aligned}
& -\left.\sum_{i \in I} \psi\right|_{a_{i}} ^{b_{i}} \cdot \varphi^{*}\left(\hat{\tau}_{i}\right)+\left.\sum_{i \in I} \psi\right|_{a_{i}} ^{b_{i}} \cdot \varphi^{*}\left(\tau_{i}^{+}\right)=\left.\sum_{i \in I} \psi\right|_{a_{i}} ^{b_{i}} \cdot \rho\left(\hat{\tau}_{i}\right) \geq 0, \\
& -\left.\sum_{i \in I} \psi\right|_{a_{i}} ^{b_{i}} \cdot \varphi^{*}\left(\hat{\tau}_{i}\right)+\left.\sum_{i \in I} \psi\right|_{a_{i}} ^{b_{i}} \cdot \varphi^{*}\left(\tau_{i}^{-}\right)=-\left.\sum_{i \in I} \psi\right|_{a_{i}} ^{b_{i}} \cdot \rho\left(\hat{\tau}_{i}\right) \geq 0,
\end{aligned}
$$

and hence $\left.\sum_{i \in I} \psi\right|_{a_{i}} ^{b_{i}} \cdot \rho\left(\hat{\tau}_{i}\right)=0$ 
Therefore, in any case, $\left.\sum_{i \in I} \psi\right|_{a_{i}} ^{b_{i}} \cdot \rho\left(\hat{\tau}_{i}\right)=0$, implying that

$$
\left.\sum_{i \in I} \psi\right|_{a_{i}} ^{b_{i}} \cdot \varphi^{*}\left(\hat{\tau}_{i}\right)=\left.\sum_{i \in I} \psi\right|_{a_{i}} ^{b_{i}} \cdot \varphi^{*}\left(\tau_{i}^{+}\right)=\left.\sum_{i \in I} \psi\right|_{a_{i}} ^{b_{i}} \cdot \varphi^{*}\left(\tau_{i}^{-}\right) .
$$

It follows that also the sequences $T^{+}=\left(\tau_{i}^{+}\right)$and $T^{-}=\left(\tau_{i}^{-}\right)$belong to $\mathcal{O}(\varphi, \psi)$. Moreover, since $\rho(t)>0$ if $t \in U$, it holds that either $h^{+}$or $h^{-}$moves every point in $U$ leftwards (according to whether $\frac{d \varphi^{*}}{d t}$ is negative or positive in $U$, respectively), while both of them do not move the points outside $U$. Therefore either $w\left(T^{+}\right)<$ $w(\widehat{T})$ or $w\left(T^{-}\right)<w(\widehat{T})$ must hold, against our hypotheses. Hence the equality $w(\widehat{T})=0$ is proved. It follows that $\widehat{\gamma}_{i}=0$ for every index $i \in \mathbb{N}$; i.e., every $\hat{\tau}_{i}$ is a critical point for $\varphi^{*}$.

Proposition 4.14 allows us to immediately obtain the next useful result, strengthening Theorem 4.9. We first state a new definition.

Definition 4.15. Let $\varphi, \psi \in A S^{1}(\mathbb{R})-\{\mathbf{0}\}$. We denote by $\mathcal{C}(\varphi, \psi)$ the set of all sequences $\left(\hat{\tau}_{i}\right) \in \mathcal{O}(\varphi, \psi)$ such that $\hat{\tau}_{i}$ is a critical point of $\varphi^{*}$ for every index $i \in I$. We shall say that these sequences are the optimal critical sequences for $(\varphi, \psi)$.

Theorem 4.16 (Representation Theorem). Let $\varphi, \psi \in A S^{1}(\mathbb{R})-\{\mathbf{0}\}$. Then

$$
\|\varphi\|_{[\psi]}=\max _{\left(\hat{\tau}_{i}\right) \in \mathcal{C}(\varphi, \psi)}\left|\sum_{i \in I} \psi\right|_{a_{i}}^{b_{i}} \cdot \varphi^{*}\left(\hat{\tau}_{i}\right) \mid .
$$

Remark 4.17. Another way of expressing Theorem 4.16 is to state that the standard RPI-norm $\|\varphi\|_{[\psi]}$ equals the value $\max _{\left(\hat{\tau}_{i}\right) \in \mathcal{C}(\varphi, \psi)}\left|\sum_{i \in I} \psi\right|_{a_{i}}^{b_{i}} \cdot \delta_{\hat{\tau}_{i}}\left(\varphi^{*}\right) \mid$. In other words, the previous Theorem 4.16 makes available an equivalent discrete definition for standard RPI-norms. This definition allows for easier computations.

We conclude this section with a remark.

Remark 4.18. If a $k$-tuple $\left(m_{0}, \ldots, m_{k-1}\right) \in \mathbb{R}^{k}$ with $m_{i} \neq 0$ for at least one index $i$ is given, then a function $\psi \in A S^{1}(\mathbb{R})-\{\mathbf{0}\}$ exists such that, for every $\varphi \in A S^{1}(\mathbb{R})$, the value $\max _{\tau_{0} \leq \tau_{1} \leq \ldots \leq \tau_{k-1}}\left|\sum_{i=0}^{k-1} m_{i} \cdot \delta_{\tau_{i}}\left(\varphi^{*}\right)\right|$ equals the standard RPI-norm $\|\varphi\|_{[\psi]}$. In fact, the Representation Theorem shows that it is sufficient to choose some points $a_{0}<b_{0}<a_{1}<b_{1}<\ldots<a_{k-1}<b_{k-1}$ and an almost sigmoidal $C^{1}$-function $\psi$ such that $\psi$ is monotone in $\left(a_{i}, b_{i}\right)$ and $\left.\psi\right|_{a_{i}} ^{b_{i}}=m_{i}$ for every $0 \leq i \leq k-1$, while $\frac{d \psi}{d t}=0$ outside the set $\bigcup_{i=0}^{k-1}\left[a_{i}, b_{i}\right]$.

In other words, this means that any finite linear combination of Dirac deltas corresponds to a standard RPI-norm. This is a partial converse of Remark 4.17, stating that any standard RPI-norm corresponds to a (not necessarily finite) linear combination of Dirac deltas. It might be interesting to know under which hypotheses the statement seen in Remark 4.18 is true for series of Dirac deltas.

\section{Relationship Between RPI-NORMS AND STANDARD RPI-NORMS}

A piecewise monotone almost sigmoidal function is an almost sigmoidal function that is monotone in each connected component of the complement of a finite set. In this section we shall prove a key result in this paper, showing that all the RPInorms of piecewise monotone $C_{c}^{1}$-functions are determined by standard RPI-norms (Theorem 5.8). 
Before dealing with the technical details of our proofs, it may be useful to sketch the underlying ideas. The basic question to be answered could be formulated in this way: "How can we use the information contained in the standard RPI-norms in order to reconstruct the function $\varphi$ ?" In order to make this point clear, let us consider, for instance, a function $\psi$ that is associated with the linear combination of Dirac deltas $\Sigma_{3}=\delta_{t_{0}}-\delta_{t_{1}}+\delta_{t_{2}}$, where the values $t_{0}, t_{1}, t_{2}$ are set equal to three suitable critical points of $\varphi^{*}$, according to the Representation Theorem 4.16. In order to get some more information about $\varphi$, we have to change $\psi$ (and consequently $\left.\Sigma_{3}\right)$. The simplest way to change $\psi$ and $\Sigma_{3}$ is to slightly perturb one of the three weights $1,-1,1$ in our linear combination of deltas. E.g., we can consider the linear combination $\Sigma_{3}^{\varepsilon}=(1+\varepsilon) \delta_{t_{0}}-\delta_{t_{1}}+\delta_{t_{2}}$, associated with a suitable function $\psi_{\varepsilon}$. For a small enough $\varepsilon$, the choice of $t_{0}, t_{1}, t_{2}$ for which $\left|\Sigma_{3}^{\varepsilon}\left(\varphi^{*}\right)\right|$ is maximum allows also $\left|\Sigma_{3}\left(\varphi^{*}\right)\right|$ to attain its maximum value. This "invariance of the basepoints $t_{0}, t_{1}, t_{2}$ with respect to small changes of the weights", and the fact that $\Sigma_{3}^{\varepsilon}\left(\varphi^{*}\right)$ and $\Sigma_{3}\left(\varphi^{*}\right)$ take the same sign, will allow us to write the following equalities:

$$
\begin{aligned}
\|\varphi\|_{\left[\psi_{\varepsilon}\right]}-\|\varphi\|_{[\psi]}=\left|\Sigma_{3}^{\varepsilon}\left(\varphi^{*}\right)\right|-\left|\Sigma_{3}\left(\varphi^{*}\right)\right| & =\varepsilon \cdot \delta_{t_{0}}\left(\varphi^{*}\right) \cdot \operatorname{sign}\left(\Sigma_{3}\left(\varphi^{*}\right)\right) \\
& =\varepsilon \cdot \varphi^{*}\left(t_{0}\right) \cdot \operatorname{sign}\left(\Sigma_{3}\left(\varphi^{*}\right)\right) .
\end{aligned}
$$

It follows that the function $\|\varphi\|_{\left[\psi_{\varepsilon}\right]}$ is differentiable with respect to $\varepsilon$ and that $\frac{d\|\varphi\|_{\left[\psi_{\varepsilon}\right]}}{d \varepsilon}(0)$ equals $\varphi^{*}\left(t_{0}\right) \cdot \operatorname{sign}\left(\Sigma_{3}\left(\varphi^{*}\right)\right)$. So, we get that the value $v_{0}$ taken by $\varphi^{*}$ at the critical point $t_{0}$ equals $\frac{d\|\varphi\|_{\left[\psi_{\varepsilon}\right]}}{d \varepsilon}(0) \cdot \operatorname{sign}\left(\Sigma_{3}\left(\varphi^{*}\right)\right)$.

We can repeat the above procedure to obtain the values taken by $\varphi^{*}$ at the other two critical points $t_{1}$ and $t_{2}$. Hence, so far, we know that $\varphi^{*}$ is a function that takes the values $v_{0}, v_{1}$ and $v_{2}$ in this order, when $t$ varies from $-\infty$ to $+\infty$. By taking functions $\psi$ with an increasing number of oscillations (i.e., $\psi$ corresponding to $\left.\sum_{i=0}^{n-1}(-1)^{i} \delta_{t_{i}}\right)$, we obtain more and more information about the values of $\varphi^{*}$ at critical points. Since $\varphi$ is piecewise monotone, if the number of oscillations of $\psi$ is large enough, then $\varphi^{*}$ is monotone between two suitable critical points $t_{i}$ and $t_{i+1}$ of $\varphi^{*}$. Obviously, we do not have enough information to locate the points $t_{i}$, but we are able to reconstruct the oscillations of $\varphi^{*}$, up to reparametrization, sign and an arbitrarily small error with respect to the variation norm. Roughly speaking, these are the ideas we are going to use.

First of all we recall the formal definition of a piecewise monotone function.

Definition 5.1. We say that $f: \mathbb{R} \rightarrow \mathbb{R}$ is piecewise monotone if a finite set $W \subset \mathbb{R}$ exists such that $f$ is monotone in each connected component of the complement of $W$. Each such a set $W$ will be said to be a separating set for $f$. If $W$ is also minimal with respect to inclusion, it will be said to be a minimal separating set for $f$. We define $l(f)$ as the minimum of the cardinalities of the separating sets for $f$.

Obviously, $l(S)=0$ and $l(\Lambda)=1$. Note that if $f \in C_{c}^{1}(\mathbb{R})$ and $f \neq \mathbf{0}$, then $l(f) \geq$ 1. It is easy to show that all minimal separating sets for $f$ take the same cardinality $l(f)$. This follows from the next simple proposition (we omit the immediate proof):

Proposition 5.2. Let $f: \mathbb{R} \rightarrow \mathbb{R}$ be a piecewise monotone function. Let $W=$ $\left\{t_{0}, \ldots, t_{m}\right\}$ and $W^{\prime}=\left\{t_{0}^{\prime}, \ldots, t_{n}^{\prime}\right\}$ be two separating sets for $f$, with $t_{0}<t_{1}<$ $\ldots<t_{m}$ and $W^{\prime}$ minimal. If for some $i$ and $j$ it holds that $t_{j}<t_{i}^{\prime}<t_{j+1}$, then $f$ is constant either in $\left[t_{j}, t_{i}^{\prime}\right]$ or in $\left[t_{i}^{\prime}, t_{j+1}\right]$. 
We also observe that the concept of piecewise monotone almost sigmoidal $C^{1}$ function is invariant under reparametrization, and that $l(\varphi)=l\left(\varphi^{*}\right)$. Moreover, the points of a minimal separating set for $\varphi^{*}$ are necessarily critical points for $\varphi^{*}$.

In the rest of this section, when $\varphi$ is a piecewise monotone almost sigmoidal $C^{1}$-function with non-empty compact support (i.e., $\varphi \neq \mathbf{0}$ ), we let $[a, b]$ denote the minimal interval containing the support of $\varphi^{*}$. Moreover, if $\left\{t_{0}, \ldots, t_{l(\varphi)-1}\right\}$ is a minimal separating set for $\varphi^{*}$, we assume it is increasingly ordered and we define $c=\min _{0 \leq i \leq l(\varphi)}\left|\varphi\left(t_{i}\right)-\varphi\left(t_{i-1}\right)\right|$, where we set $t_{-1}=a$ and $t_{l(\varphi)}=b$. This meaning of the symbols $t_{-1}$ and $t_{l(\varphi)}$ will be maintained in the following pages.

Before proceeding, we need to introduce a new family of functions.

Definition 5.3. Let $n \geq 1$. For every vector $e=\left(\varepsilon_{0}, \ldots, \varepsilon_{n-1}\right) \in \mathbb{R}^{n}$ we define the functions $S_{n}, S_{n}^{e}: \mathbb{R} \rightarrow \mathbb{R}$ by setting $S_{n}(t)=\sum_{i=0}^{n-1}(-1)^{i} S(t-2 i)$ and $S_{n}^{e}(t)=$ $\sum_{i=0}^{n-1}\left((-1)^{i}+\varepsilon_{i}\right) S(t-2 i)$.

In plain words, the function $S_{n}^{e}$ is a perturbation of the function $S_{n}$. In particular, for $e=(0, \ldots, 0)$, the function $S_{n}^{e}$ equals the function $S_{n}$. We observe that the functions $S_{n}^{e}$ are piecewise polynomial and belong to $A S^{1}(\mathbb{R})$. We also note that $\|\varphi\|_{\left[S_{n}\right]} \leq\|\varphi\|_{\left[S_{n+1}\right]}$ for every $n \geq 1$ and every $\varphi \in A S^{1}(\mathbb{R})$.

The following lemma is a key passage towards the proof of the Reconstruction Theorem 5.8 for piecewise monotone functions in $C_{c}^{1}(\mathbb{R})$.

Lemma 5.4. Let $\varphi \neq \mathbf{0}$ be a piecewise monotone $C^{1}$-function with compact support. Let $[a, b]$ denote the minimal interval containing the support of $\varphi^{*}$. If $\left\{t_{0}, \ldots\right.$, $\left.t_{l(\varphi)-1}\right\}$ is an (increasingly ordered) minimal separating set for $\varphi^{*}$ and $c=$ $\min _{0 \leq i \leq l(\varphi)}\left|\varphi\left(t_{i}\right)-\varphi\left(t_{i-1}\right)\right|\left(\right.$ where we set $t_{-1}=a$ and $\left.t_{l(\varphi)}=b\right)$, then

(1) $\|\varphi\|_{\left[S_{n}\right]}=\left|\sum_{i=0}^{l(\varphi)-1}(-1)^{i} \cdot \varphi^{*}\left(t_{i}\right)\right|=\frac{1}{2} \cdot V_{\varphi}$ for any $n \geq l(\varphi)$;

(2) $\|\varphi\|_{\left[S_{n}\right]} \leq\|\varphi\|_{\left[S_{l(\varphi)}\right]}-c$, for $1 \leq n<l(\varphi)$.

Proof. We start by proving (1). Let us consider the finite set $\mathcal{J}\left(S_{n}\right)$ of all maximal open intervals of $\mathbb{R}$ where $\frac{d S_{n}}{d t}$ does not vanish. We note that $\mathcal{J}\left(S_{n}\right)=\left\{J_{i}\right\}_{i \in I}$, where $J_{i}=(-1+2 i, 1+2 i), I=\{0, \ldots, n-1\}$, and $\left.S_{n}\right|_{-1+2 i} ^{1+2 i}=(-1)^{i}$.

From the Bounding Lemma 2.17for functions in $C_{c}^{1}(\mathbb{R})$, we obtain that $\|\varphi\|_{\left[S_{n}\right]} \leq$ $V_{\varphi} / 2$ (we point out that Remark 4.17 implies $\|\Lambda\|_{\left[S_{n}\right]}=1$ ). Observing that $\left|\sum_{i=0}^{l(\varphi)-1}(-1)^{i} \cdot \varphi^{*}\left(t_{i}\right)\right|=V_{\varphi} / 2$, we easily conclude that $\left(t_{0}, \ldots, t_{l(\varphi)-1}, b, b, b, \ldots\right)$ is an optimal sequence for $\left(\varphi, S_{n}\right)$, and that $\|\varphi\|_{\left[S_{n}\right]}=V_{\varphi} / 2$ for any $n \geq l(\varphi)$.

We now prove $(2)$. Let $1 \leq n<l(\varphi)$. Since $\|\varphi\|_{\left[S_{n}\right]} \leq\|\varphi\|_{\left[S_{n+1}\right]}$, it is sufficient to prove that $\|\varphi\|_{\left[S_{l(\varphi)-1}\right]} \leq\|\varphi\|_{\left[S_{l(\varphi)}\right]}-c$.

If $l(\varphi)=2$, then $\max \varphi \geq c,-\min \varphi \geq c$ so that

$$
\|\varphi\|_{\left[S_{l(\varphi)}\right]}-\|\varphi\|_{\left[S_{l(\varphi)-1}\right]}=\|\varphi\|_{[\Lambda]}-\|\varphi\|_{[S]}=\max \varphi-\min \varphi-\max |\varphi| \geq c .
$$

Let us now assume that $l(\varphi) \geq 3$. Let $T=\left(\tau_{i}\right)$ be an optimal sequence for $\left(\varphi, S_{l(\varphi)-1}\right)$, increasingly ordered so that $\|\varphi\|_{\left[S_{l(\varphi)-1}\right]}=\left|\sum_{i=0}^{l(\varphi)-2}(-1)^{i} \cdot \varphi^{*}\left(\tau_{i}\right)\right|$. The key point of the proof relies on understanding where to place $\tau_{0}, \tau_{1}, \ldots, \tau_{l(\varphi)-2}$, in order to achieve optimality. 
First of all, we can assume that

i): the only repeated point in the sequence $T$ is $b$

since consecutive points appear with opposite weights, and that

ii): each $\tau_{i}$ belongs to $\left\{t_{0}, t_{1}, \ldots, t_{l(\varphi)-1}\right\} \cup\{a, b\}$.

Indeed, if statement ii) were false we could easily find a better sequence than $T$ by using the monotonicity of $\varphi^{*}$ outside the set $\left\{t_{0}, t_{1}, \ldots, t_{l(\varphi)-1}\right\}$, and hence $T$ would not be optimal.

Now we note that $\tau_{l(\varphi)-1}=b$, since $\tau_{l(\varphi)-1}$ is the first dummy point of $T \in$ $\mathcal{T}\left(\varphi, S_{l(\varphi)-1}\right)$ (recall Definition 4.6). Therefore, if $\tau_{0}=a$ we obtain

$$
\sum_{i=0}^{l(\varphi)-2}(-1)^{i} \cdot \varphi^{*}\left(\tau_{i}\right)=-\sum_{i=0}^{l(\varphi)-2}(-1)^{i} \cdot \varphi^{*}\left(\tau_{i+1}\right)
$$

because $\varphi^{*}(a)=\varphi^{*}(b)=0$. Hence, possibly by replacing the optimal sequence $\left(\tau_{i}\right)$ with the new optimal sequence $\left(\tau_{i+1}\right)$, we can substitute ii) with

iii): each $\tau_{i}$ belongs to $\left\{t_{0}, t_{1}, \ldots, t_{l(\varphi)-1}\right\} \cup\{b\}$.

Possibly changing $\varphi$ with $-\varphi$, we can assume that

iv): $\sum_{i=0}^{l(\varphi)-2}(-1)^{i} \cdot \varphi^{*}\left(\tau_{i}\right) \geq 0$.

Now two mutually exclusive cases are possible:

A): $\varphi^{*}$ is increasing in $\left[a, t_{0}\right]$;

B): $\varphi^{*}$ is decreasing in $\left[a, t_{0}\right]$.

We can prove the following property (in the following we shall set $t_{l(\varphi)}=b$ ):

$\mathbf{v - A ) : ~ I n ~ c a s e ~} \boldsymbol{A})$, for each $i$ with $0 \leq i \leq l(\varphi)-2$ exactly one index $j$ exists such that $0 \leq j \leq l(\varphi)$ and $\tau_{i}=t_{j}$. Furthermore, $i-j$ is even.

The new statement is that $i-j$ is even. In order to prove $\mathbf{v - A}$ ) by contradiction, let us assume that $i$ is even and $j$ is odd. Since $\mathbf{A}$ ) holds and $j$ is odd, $\varphi^{*}$ is decreasing in $\left[t_{j-1}, t_{j}\right]$ (this follows from the definition of minimal separating set). Although $\varphi^{*}$ may not be strictly decreasing in $\left[t_{j-1}, t_{j}\right]$, the definition of minimal separating set for $\varphi^{*}$ implies that $\varphi^{*}\left(t_{j}\right)<\varphi^{*}\left(t_{j-1}\right)$. Hence $(-1)^{i} \cdot \varphi^{*}\left(\tau_{i}\right)=\varphi^{*}\left(t_{j}\right)<$ $\varphi^{*}\left(t_{j-1}\right)=(-1)^{i} \cdot \varphi^{*}\left(t_{j-1}\right)$. Now, let us assume that $i$ is odd and $j$ is even. Since A) holds and $j$ is even, $\varphi^{*}$ is increasing in $\left[t_{j-1}, t_{j}\right]$. Hence $(-1)^{i} \cdot \varphi^{*}\left(\tau_{i}\right)=-\varphi^{*}\left(t_{j}\right)<$ $-\varphi^{*}\left(t_{j-1}\right)=(-1)^{i} \cdot \varphi^{*}\left(t_{j-1}\right)$. Therefore, if $i$ and $j$ did not have the same parity, then the sequence $T$ would not be optimal, since $(-1)^{i} \cdot \varphi^{*}\left(\tau_{i}\right)<(-1)^{i} \cdot \varphi^{*}\left(t_{j-1}\right)$ and we could obtain a better sequence by redefining $\tau_{i}=t_{j-1}$. This proves that $i-j$ is even in case $\mathbf{A}$ ).

Moreover, we can prove the following property (once again, we shall set $t_{l(\varphi)}=b$ ):

$\mathbf{v - B ) : ~ I n ~ c a s e ~} \boldsymbol{B})$, for each $i$ with $0 \leq i \leq l(\varphi)-2$, exactly one index $j$ exists such that $0 \leq j \leq l(\varphi)$ and $\tau_{i}=t_{j}$. Furthermore, $i-j$ is odd.

The new statement is that $i-j$ is odd. In order to prove $\mathbf{v - B}$ ) by contradiction, let us assume that both $i$ and $j$ are even. Since B) holds and $j$ is even, $\varphi^{*}$ is decreasing in $\left[t_{j-1}, t_{j}\right]$ (this follows from the definition of minimal separating set). Although $\varphi^{*}$ may not be strictly decreasing in $\left[t_{j-1}, t_{j}\right]$, the definition of minimal separating set for $\varphi^{*}$ implies that $\varphi^{*}\left(t_{j}\right)<\varphi^{*}\left(t_{j-1}\right)$. Hence $(-1)^{i} \cdot \varphi^{*}\left(\tau_{i}\right)=\varphi^{*}\left(t_{j}\right)<$ $\varphi^{*}\left(t_{j-1}\right)=(-1)^{i} \cdot \varphi^{*}\left(t_{j-1}\right)$. Now, let us assume that both $i$ and $j$ are odd. Since $\left.\mathbf{B}\right)$ holds and $j$ is odd, $\varphi^{*}$ is increasing in $\left[t_{j-1}, t_{j}\right]$. Hence $(-1)^{i} \cdot \varphi^{*}\left(\tau_{i}\right)=-\varphi^{*}\left(t_{j}\right)<$ $-\varphi^{*}\left(t_{j-1}\right)=(-1)^{i} \cdot \varphi^{*}\left(t_{j-1}\right)$. Therefore, if $i$ and $j$ had the same parity, the sequence 
$T$ would not be optimal, since $(-1)^{i} \cdot \varphi^{*}\left(\tau_{i}\right)<(-1)^{i} \cdot \varphi^{*}\left(t_{j-1}\right)$, and we could obtain a better sequence by redefining $\tau_{i}=t_{j-1}$. This proves that $i-j$ is odd in case $\mathbf{B}$ ).

Properties v-A) and v-B) imply that both in case $\mathbf{A}$ ) and in case $\mathbf{B}$ ),

$\mathbf{v})$ : for each $i$ with $0 \leq i \leq l(\varphi)-2$, exactly one index $j$ exists such that $0 \leq j \leq l(\varphi)$ and $\tau_{i}=t_{j}$. Furthermore, if $i$ is even, then $\varphi^{*}\left(t_{j}\right)>\varphi^{*}\left(t_{j-1}\right)$, while if $i$ is odd, then $\varphi^{*}\left(t_{j}\right)<\varphi^{*}\left(t_{j-1}\right)$ (here we set $t_{-1}=a$ ).

Indeed, if $i$ is even and $\mathbf{A}$ ) holds, property $\mathbf{v}-\mathbf{A}$ ) implies that $j$ is even, so that $\varphi^{*}$ is increasing in $\left[t_{j-1}, t_{j}\right]$. If $i$ is even and $\mathbf{B}$ ) holds, property $\mathbf{v}-\mathbf{B}$ ) implies that $j$ is odd, so that $\varphi^{*}$ is increasing in $\left[t_{j-1}, t_{j}\right]$. If $i$ is odd and $\mathbf{A}$ ) holds, property $\mathbf{v - A}$ ) implies that $j$ is odd, so that $\varphi^{*}$ is decreasing in $\left[t_{j-1}, t_{j}\right]$. If $i$ is odd and $\mathbf{B}$ ) holds, property $\mathbf{v - B}$ ) implies that $j$ is even, so that $\varphi^{*}$ is decreasing in $\left[t_{j-1}, t_{j}\right]$. In summary, if $i$ is even, then $\varphi^{*}$ is increasing in $\left[t_{j-1}, t_{j}\right]$, while if $i$ is odd, then $\varphi^{*}$ is decreasing in $\left[t_{j-1}, t_{j}\right]$. This proves property $\left.\mathbf{v}\right)$.

Now we can prove that

vi): $\tau_{l(\varphi)-3} \neq b$ (and hence $\tau_{i} \neq b$ for every $i \leq l(\varphi)-3$ ).

In order to check vi), let us proceed by contradiction and assume that $\tau_{l(\varphi)-3}=b$. Note that necessarily $\tau_{l(\varphi)-2}=\tau_{l(\varphi)-1}=b$. Since the number of points $\tau_{i}$ that are different from $b$ is at most $l(\varphi)-3$, at least three points of the separating set $\left\{t_{0}, t_{1}, \ldots, t_{l(\varphi)-1}\right\}$ do not coincide with any $\tau_{i}$. Let us consider the largest index $k \leq l(\varphi)-1$ such that $t_{k}$ does not belong to the set $\left\{\tau_{i}\right\}$. Since, by definition, $\left.t_{k+1} \in\left\{\tau_{i}\right\}, \mathbf{v}-\mathbf{A}\right)$ and $\left.\mathbf{v}-\mathbf{B}\right)$ imply that $t_{k-1}$ does not belong to the set $\left\{\tau_{i}\right\}$. Indeed, properties $\mathbf{v}-\mathbf{A}$ ) and $\mathbf{v}-\mathbf{B}$ ) guarantee that, for every index $i$, the points $\tau_{i}$ and $\tau_{i+1}$ are separated by an even number of points $t_{j}$ (possibly 0 ). Let us define $\bar{\iota}=\min \left\{i: \tau_{i}=t_{k+1}\right\}$ (we set $t_{l(\varphi)}=b$ ) and consider the sequence $T^{\prime}=\left(\tau_{i}^{\prime}\right.$ ) obtained by setting $\tau_{i}^{\prime}=\tau_{i}$ if $i<\bar{\iota}, \tau_{\bar{\iota}}^{\prime}=t_{k-1}, \tau_{\bar{\iota}+1}^{\prime}=t_{k}$ and $\tau_{i}^{\prime}=\tau_{i-2}$ if $i \geq \bar{\iota}+2$. In other words, $T^{\prime}$ differs from $T$ by the insertion of the pair of points $t_{k-1}$ and $t_{k}$. Since $\tau_{\bar{\iota}}=t_{k+1}$ with $\bar{\iota} \leq l(\varphi)-3$, by applying $\left.\mathbf{v}\right)$ we have $\varphi^{*}\left(t_{k+1}\right)>\varphi^{*}\left(t_{k}\right)$ if $\bar{\iota}$ is even, and $\varphi^{*}\left(t_{k+1}\right)<\varphi^{*}\left(t_{k}\right)$ if $\bar{\iota}$ is odd. As a consequence, $\varphi^{*}\left(t_{k-1}\right)>\varphi^{*}\left(t_{k}\right)$ if $\bar{\iota}$ is even, and $\varphi^{*}\left(t_{k-1}\right)<\varphi^{*}\left(t_{k}\right)$ if $\bar{\iota}$ is odd. Therefore, both for $\bar{\iota}$ even and for $\bar{\iota}$ odd we get

$$
(-1)^{\bar{\iota}} \varphi^{*}\left(\tau_{\bar{\iota}}^{\prime}\right)+(-1)^{\bar{\tau}+1} \varphi^{*}\left(\tau_{\bar{\iota}+1}^{\prime}\right)=(-1)^{\bar{\iota}}\left(\varphi^{*}\left(t_{k-1}\right)-\varphi^{*}\left(t_{k}\right)\right)>0 .
$$

Therefore, we have that

$$
\begin{aligned}
\sum_{i=0}^{l(\varphi)-2}(-1)^{i} \cdot \varphi^{*}\left(\tau_{i}\right) & =\sum_{i=0}^{\bar{l}-1}(-1)^{i} \cdot \varphi^{*}\left(\tau_{i}\right)+\sum_{i=\bar{L}}^{l(\varphi)-2}(-1)^{i} \cdot \varphi^{*}\left(\tau_{i}\right) \\
& =\sum_{i=0}^{\bar{l}-1}(-1)^{i} \cdot \varphi^{*}\left(\tau_{i}^{\prime}\right)+\sum_{i=\bar{\iota}+2}^{l(\varphi)}(-1)^{i} \cdot \varphi^{*}\left(\tau_{i}^{\prime}\right) \\
& <\sum_{i=0}^{l(\varphi)}(-1)^{i} \cdot \varphi^{*}\left(\tau_{i}^{\prime}\right),
\end{aligned}
$$

contradicting the optimality of $T$. Hence property vi) is proved.

The previous property vi) shows that $\left\{\tau_{0}, \tau_{1}, \ldots, \tau_{l(\varphi)-3}\right\} \subseteq\left\{t_{0}, t_{1}, \ldots, t_{l(\varphi)-1}\right\}$, and hence exactly $l(\varphi)-2$ points in the separating set must belong to the set 
$\left\{\tau_{0}, \tau_{1}, \ldots, \tau_{l(\varphi)-3}\right\}$. From all this we deduce that the only dispositions allowed for $\tau_{0}, \tau_{1}, \ldots, \tau_{l(\varphi)-2}$ are the following ones:

(1) $\tau_{0}=t_{1}, \tau_{1}=t_{2}, \ldots, \tau_{l(\varphi)-2}=t_{l(\varphi)-1}$;

(2) $\tau_{0}=t_{0}, \tau_{1}=t_{1}, \ldots, \tau_{l(\varphi)-2}=t_{l(\varphi)-2}$;

(3) an index $k$ with $1 \leq k \leq l(\varphi)-1$ exists such that $\left\{t_{0}, \ldots, t_{l(\varphi)-1}\right\}=$ $\left\{\tau_{0}, \ldots, \tau_{l(\varphi)-3}\right\} \cup\left\{t_{k-1}, t_{k}\right\}$.

The first two cases happen when $\tau_{l(\varphi)-2} \neq b$, the last case when $\tau_{l(\varphi)-2}=b$. Note that, if $\tau_{l(\varphi)-2} \neq b$, exactly $l(\varphi)-1$ points in the separating set must belong to the set $\left\{\tau_{0}, \tau_{1}, \ldots, \tau_{l(\varphi)-2}\right\}$, and recall once again that, for every index $i$, the points $\tau_{i}$ and $\tau_{i+1}$ are separated by an even number of points $t_{j}$ (possibly 0 ). Hence the only $t_{j}$ 's that can be missing in the set $\left\{\tau_{0}, \tau_{1}, \ldots, \tau_{l(\varphi)-2}\right\}$ are $t_{0}$ and $t_{l(\varphi)-1}$. This observation produces the cases (1) and (2). If $\tau_{l(\varphi)-2}=b$, a gap of two consecutive $t_{j}$ 's is possible, implying case (3).

In the first case, $\operatorname{since} \operatorname{sign}\left(\varphi^{*}\left(t_{0}\right)\right)=\operatorname{sign}\left(\sum_{i=0}^{l(\varphi)-1}(-1)^{i} \cdot \varphi^{*}\left(t_{i}\right)\right)$ (we can easily verify this equality both in cases A) and B)) and $c \leq\left|\varphi^{*}\left(t_{0}\right)\right| \leq\|\varphi\|_{\left[S_{1}\right]} \leq\|\varphi\|_{\left[S_{l(\varphi)}\right]}$, we have that

$$
\begin{aligned}
\|\varphi\|_{\left[S_{l(\varphi)-1}\right]} & =\left|\sum_{i=0}^{l(\varphi)-2}(-1)^{i} \cdot \varphi^{*}\left(\tau_{i}\right)\right|=\left|\sum_{i=1}^{l(\varphi)-1}(-1)^{i} \cdot \varphi^{*}\left(t_{i}\right)\right| \\
& =\left|\sum_{i=0}^{l(\varphi)-1}(-1)^{i} \cdot \varphi^{*}\left(t_{i}\right)-\varphi^{*}\left(t_{0}\right)\right| \\
& =|| \sum_{i=0}^{l(\varphi)-1}(-1)^{i} \cdot \varphi^{*}\left(t_{i}\right)|-| \varphi^{*}\left(t_{0}\right)|=|\|\varphi\|_{\left[S_{l(\varphi)}\right]}-\left|\varphi^{*}\left(t_{0}\right)\right| \mid \\
& =\|\varphi\|_{\left[S_{l(\varphi)}\right]}-\left|\varphi^{*}\left(t_{0}\right)\right| \leq\|\varphi\|_{\left[S_{l(\varphi)}\right]}-c .
\end{aligned}
$$

In the second case, the claim is proved analogously, since $c \leq\left|\varphi^{*}\left(t_{l(\varphi)-1}\right)\right| \leq$ $\|\varphi\|_{\left[S_{1}\right]} \leq\|\varphi\|_{\left[S_{l(\varphi)}\right]}$ and

$$
\operatorname{sign}\left((-1)^{l(\varphi)-1} \varphi^{*}\left(t_{l(\varphi)-1}\right)\right)=\operatorname{sign}\left(\sum_{i=0}^{l(\varphi)-1}(-1)^{i} \cdot \varphi^{*}\left(t_{i}\right)\right)
$$

This last equality immediately follows from the equality

$$
\operatorname{sign}\left(\varphi^{*}\left(t_{0}\right)\right)=\operatorname{sign}\left(\sum_{i=0}^{l(\varphi)-1}(-1)^{i} \cdot \varphi^{*}\left(t_{i}\right)\right)
$$

by replacing the function $\varphi^{*}(t)$ with the function $(-1)^{l(\varphi)-1} \varphi^{*}(-t)$ and inverting the order of the points in the separating set. 
Therefore, in case (2), we have that

$$
\begin{aligned}
& \|\varphi\|_{\left[S_{l(\varphi)-1}\right]}=\left|\sum_{i=0}^{l(\varphi)-2}(-1)^{i} \cdot \varphi^{*}\left(\tau_{i}\right)\right|=\left|\sum_{i=0}^{l(\varphi)-2}(-1)^{i} \cdot \varphi^{*}\left(t_{i}\right)\right| \\
& =\left|\sum_{i=0}^{l(\varphi)-1}(-1)^{i} \cdot \varphi^{*}\left(t_{i}\right)-(-1)^{l(\varphi)-1} \varphi^{*}\left(t_{l(\varphi)-1}\right)\right| \\
& =|| \sum_{i=0}^{l(\varphi)-1}(-1)^{i} \cdot \varphi^{*}\left(t_{i}\right)|-| \varphi^{*}\left(t_{l(\varphi)-1}\right) \mid \leq\|\varphi\|_{\left[S_{l(\varphi)}\right]}-c .
\end{aligned}
$$

Finally, in case (3),

$$
\begin{aligned}
\|\varphi\|_{\left[S_{l(\varphi)-1}\right]} & =\left|\sum_{i=0}^{l(\varphi)-2}(-1)^{i} \cdot \varphi^{*}\left(\tau_{i}\right)\right| \\
& =\left|\sum_{i=0}^{l(\varphi)-1}(-1)^{i} \cdot \varphi^{*}\left(t_{i}\right)-\left((-1)^{k-1} \varphi^{*}\left(t_{k-1}\right)+(-1)^{k} \varphi^{*}\left(t_{k}\right)\right)\right| \\
& =|| \sum_{i=0}^{l(\varphi)-1}(-1)^{i} \cdot \varphi^{*}\left(t_{i}\right)|-|(-1)^{k-1} \varphi^{*}\left(t_{k-1}\right)+(-1)^{k} \varphi^{*}\left(t_{k}\right) \mid \\
& \leq\|\varphi\|_{\left[S_{l(\varphi)}\right]}-c,
\end{aligned}
$$

because

$$
\operatorname{sign}\left((-1)^{k-1} \varphi^{*}\left(t_{k-1}\right)+(-1)^{k} \varphi^{*}\left(t_{k}\right)\right)=\operatorname{sign}\left(\sum_{i=0}^{l(\varphi)-1}(-1)^{i} \cdot \varphi^{*}\left(t_{i}\right)\right)
$$

(as can easily be verified both in case $\mathbf{A}$ ) and case $\mathbf{B}$ )) and

$$
c \leq\left|(-1)^{k-1} \varphi^{*}\left(t_{k-1}\right)+(-1)^{k} \varphi^{*}\left(t_{k}\right)\right| \leq\|\varphi\|_{\left[S_{2}\right]} \leq\|\varphi\|_{\left[S_{l(\varphi)}\right]} .
$$

An immediate consequence of the previous lemma is the following result, allowing us to deduce the value of $l(\varphi)$ from the knowledge of the standard RPI-norms $\|\varphi\|_{\left[S_{n}\right]}, n \geq 1$, when $\varphi$ is piecewise monotone and belongs to $C_{c}^{1}(\mathbb{R})$.

Corollary 5.5. Let $\varphi \neq 0$ be a piecewise monotone $C^{1}$-function with compact support. The value $l(\varphi)$ is equal to the smallest integer $N$ such that $\|\varphi\|_{\left[S_{N}\right]}=$ $\|\varphi\|_{\left[S_{n}\right]}$ for every $n \geq N$.

In the following lemma, we consider the pairs $\left(\varphi, S_{l(\varphi)}^{e}\right)$ varying $e$, and we show that for every small enough $e$, they all admit the same optimal sequence.

Lemma 5.6. Let $\varphi \neq \mathbf{0}$ be a piecewise monotone $C^{1}$-function with compact support. Let $W=\left\{t_{0}, \ldots, t_{l(\varphi)-1}\right\}$ be an (increasingly ordered) minimal separating set for 
$\varphi^{*}$. For every $e=\left(\varepsilon_{0}, \ldots, \varepsilon_{l(\varphi)-1}\right) \in \mathbb{R}^{l(\varphi)}$ with $\max _{i}\left|\varepsilon_{i}\right|<\min \left\{\frac{c}{2 \cdot l(\varphi) \cdot \max |\varphi|}, 1\right\}$, it holds that $\left(t_{0}, \ldots, t_{l(\varphi)-1}, b, b, b, \ldots\right)$ is an optimal sequence for $\left(\varphi, S_{l(\varphi)}^{e}\right)$, i.e.,

$$
\left.\left|\sum_{i=0}^{l(\varphi)-1}\left((-1)^{i}+\varepsilon_{i}\right) \cdot \varphi^{*}\left(t_{i}\right)\right|=\|\varphi\|_{\left[S_{l(\varphi)}^{e}\right]}\right]
$$

Proof. First of all we note that $c>0$ by definition. Let us consider the finite set $\mathcal{J}\left(S_{l(\varphi)}^{e}\right)$ of all maximal open intervals of $\mathbb{R}$ where $\frac{d S_{l(\varphi)}^{e}}{d t}$ does not vanish. Since $\max \left|\varepsilon_{i}\right|<1$, we have that $\mathcal{J}\left(S_{l(\varphi)}^{e}\right)=\left\{J_{i}\right\}_{i \in I}$, where $J_{i}=(-1+2 i, 1+2 i)$, $I=\{0, \ldots, l(\varphi)-1\}$, and $\left.S_{l(\varphi)}^{e}\right|_{-1+2 i} ^{1+2 i}=(-1)^{i}+\varepsilon_{i}$.

Consider an optimal sequence for $\left(\varphi, S_{l(\varphi)}^{e}\right), T=\left(\tau_{0}, \ldots, \tau_{l(\varphi)-1}, b, b, b, \ldots\right)$ (increasingly ordered). As we have already done in the proof of Lemma 5.4. we can assume that $\left\{\tau_{i}\right\} \subseteq W \cup\{a, b\}$, and that the only repeated point in $T$ is $b$. We claim that, necessarily, $\left(\tau_{0}, \ldots, \tau_{l(\varphi)-1}\right)=\left(t_{0}, \ldots, t_{l(\varphi)-1}\right)$. Otherwise, either at least one point at the beginning of $\left(\tau_{0}, \ldots, \tau_{l(\varphi)-1}\right)$ is equal to $a$, or at least one point at the end of the same $l(\varphi)$-tuple is equal to $b$. In this case, $\left\{\tau_{0}, \ldots, \tau_{l(\varphi)-1}\right\}-\{a, b\}$ is properly included in $\left\{t_{0}, \ldots, t_{l(\varphi)-1}\right\}$, so that $\left|\sum_{i=0}^{l(\varphi)-1}(-1)^{i} \varphi^{*}\left(\tau_{i}\right)\right| \leq\|\varphi\|_{\left[S_{l(\varphi)-1}\right]}$, since $\varphi^{*}(a)=\varphi^{*}(b)=0$. Hence Lemma 5.4 implies that

$$
\begin{aligned}
\left|\sum_{i=0}^{l(\varphi)-1}(-1)^{i} \varphi^{*}\left(t_{i}\right)\right|-\left|\sum_{i=0}^{l(\varphi)-1}(-1)^{i} \varphi^{*}\left(\tau_{i}\right)\right| & =\|\varphi\|_{\left[S_{l(\varphi)}\right]}-\left|\sum_{i=0}^{l(\varphi)-1}(-1)^{i} \varphi^{*}\left(\tau_{i}\right)\right| \\
& \geq\|\varphi\|_{\left[S_{l(\varphi)}\right]}-\|\varphi\|_{\left[S_{l(\varphi-1)}\right]} \geq c
\end{aligned}
$$

and thus

$$
\begin{array}{r}
\left|\sum_{i=0}^{l(\varphi)-1}\left((-1)^{i}+\varepsilon_{i}\right) \cdot \varphi^{*}\left(t_{i}\right)\right|-\left|\sum_{i=0}^{l(\varphi)-1}\left((-1)^{i}+\varepsilon_{i}\right) \cdot \varphi^{*}\left(\tau_{i}\right)\right| \\
\geq c-2 \cdot l(\varphi) \cdot \max |\varphi| \cdot \max _{i}\left|\varepsilon_{i}\right|>0,
\end{array}
$$

contradicting the optimality of the sequence $T$.

We now show that the standard RPI-norms of $\varphi$, with respect to $S_{l(\varphi)}^{e}$, allow us to obtain the values of $\varphi$ at the points of each minimal separating set.

Lemma 5.7. Let $\varphi \neq \mathbf{0}$ be a piecewise monotone $C^{1}$-function with compact support. Let us consider an (increasingly ordered) minimal separating set $W=\left\{t_{0}, \ldots\right.$, $\left.t_{l(\varphi)-1}\right\}$ for $\varphi^{*}$. For every $e=\left(\varepsilon_{0}, \ldots, \varepsilon_{l(\varphi)-1}\right) \in \mathbb{R}^{l(\varphi)}$ with $\max _{i}\left|\varepsilon_{i}\right|$ $<\min \left\{\frac{c}{2 \cdot l(\varphi) \cdot \max |\varphi|}, 1\right\}$ it holds that

(1) the function $\|\varphi\|_{\left[S_{l(\varphi)}^{e}\right]}$ is differentiable in the variables $\varepsilon_{0}, \ldots, \varepsilon_{l(\varphi)-1}$;

(2) $\varphi^{*}\left(t_{i}\right)=s \cdot \frac{\partial}{\partial \varepsilon_{i}}\|\varphi\|_{\left[S_{l(\varphi)}^{e}\right]}(0)$ for $0 \leq i \leq l(\varphi)-1$, where $s$ is the sign of $\sum_{i=0}^{l(\varphi)-1}(-1)^{i} \cdot \varphi^{*}\left(t_{i}\right)$. 
Proof. From Lemma 5.6 it follows that for every $e=\left(\varepsilon_{0}, \ldots, \varepsilon_{l(\varphi)-1}\right) \in \mathbb{R}^{l(\varphi)}$ with $\left|\varepsilon_{0}\right|, \ldots,\left|\varepsilon_{l(\varphi)-1}\right|<\min \left\{\frac{c}{2 \cdot l(\varphi) \cdot \max |\varphi|}, 1\right\}$ it holds that $\|\varphi\|_{\left[S_{l(\varphi)}^{e}\right]}=$ $\left|\sum_{i=0}^{l(\varphi)-1}\left((-1)^{i}+\varepsilon_{i}\right) \cdot \varphi^{*}\left(t_{i}\right)\right|$. As a consequence, the function $\|\varphi\|_{\left[S_{l(\varphi)}^{e}\right]}$ is differentiable in each variable $\varepsilon_{i}$ and

$$
\frac{\partial}{\partial \varepsilon_{i}}\|\varphi\|_{\left[S_{l(\varphi)}^{e}\right]}(0)=s \cdot \varphi^{*}\left(t_{i}\right)
$$

for $0 \leq i \leq l(\varphi)-1$, where $s$ is the sign of $\sum_{i=0}^{l(\varphi)-1}(-1)^{i} \cdot \varphi^{*}\left(t_{i}\right)$ (note that $s$ does not depend on $e$ ).

We are now ready to prove that given a piecewise monotone $C^{1}$-function $\varphi$ with compact support, it is possible to construct a piecewise polynomial almost sigmoidal $C^{1}$-function $\hat{\varphi}$, such that $\hat{\varphi}$ approximates $\varphi$ in the total variation norm up to reparametrization and sign, and $\|\varphi\|=\|\hat{\varphi}\|$ for any RPI-norm on $A S^{1}(\mathbb{R})$. The key point here is that this construction is based just on the knowledge of the values taken by the standard RPI-norms at $\varphi$. In other words, the RPI-norms of piecewise monotone functions in $C_{c}^{1}(\mathbb{R})$ are determined by the standard RPI-norms.

Theorem 5.8 (Reconstruction Theorem for piecewise monotone functions in $\left.C_{c}^{1}(\mathbb{R})\right)$. Assume that $\varphi$ is a piecewise monotone $C^{1}$-function with compact support. Let $e=\left(\varepsilon_{0}, \ldots, \varepsilon_{l(\varphi)-1}\right) \in \mathbb{R}^{l(\varphi)}$, where $\left|\varepsilon_{0}\right|, \ldots,\left|\varepsilon_{l(\varphi)-1}\right| \leq \varepsilon$. If $0<\varepsilon<$ $\min \left\{\frac{c}{2 \cdot l(\varphi) \cdot \max |\varphi|}, 1\right\}$, we can define the piecewise polynomial $C^{1}$-function $\widehat{\varphi} \in$ $A S^{1}(\mathbb{R})$ by setting

$\widehat{\varphi}(t)=\frac{\partial\|\varphi\|_{\left[S_{l(\varphi)}^{e}\right]}}{\partial \varepsilon_{l(\varphi)-1}}(0) \cdot S(t)+\sum_{j=1}^{l(\varphi)-1}\left(\frac{\partial\|\varphi\|_{\left[S_{l(\varphi)}^{e}\right]}}{\partial \varepsilon_{l(\varphi)-j-1}}(0)-\frac{\partial\|\varphi\|_{\left[S_{l(\varphi)}^{e}\right]}}{\partial \varepsilon_{l(\varphi)-j}}(0)\right) \cdot S(t-2 j)$,

so that the following statements hold:

a): there exists an orientation-preserving $C^{1}$-diffeomorphism $h: \mathbb{R} \rightarrow \mathbb{R}$ such that either $V_{\varphi \circ h-\widehat{\varphi}} \leq \varepsilon$ or $V_{-\varphi \circ h-\widehat{\varphi}} \leq \varepsilon ;$

b): $\|\varphi\|=\|\hat{\varphi}\|$ for any RPI-norm on $A S^{1}(\mathbb{R})$.

Proof. Let us assume $\varphi \neq \mathbf{0}$; otherwise, the claims are trivial. Let $\left\{t_{0}, t_{1}, \ldots\right.$, $\left.t_{l(\varphi)-1}\right\}$ be a minimal separating set for $\varphi^{*}$, with $t_{0}<t_{1}<\ldots<t_{l(\varphi)-1}$. By applying Lemma [5.7, for $i=0, \ldots, l(\varphi)-1, \varphi^{*}\left(t_{i}\right)=s \cdot \frac{\partial\|\varphi\|_{\left[S_{l(\varphi)}^{e}\right]}}{\partial \varepsilon_{i}}(0)$, with $s=\operatorname{sign}\left(\sum_{i=0}^{l(\varphi)-1}(-1)^{i} \cdot \varphi^{*}\left(t_{i}\right)\right)$. Thus, the norms $\|\varphi\|_{\left[S_{l(\varphi)}^{e}\right]}$ (varying $e$ ) allow us to determine, up to the sign, the values of $\varphi^{*}$ at the points $t_{0}, t_{1}, \ldots, t_{l(\varphi)-1}$. Furthermore, we have that

$$
s \cdot \widehat{\varphi}(t)=\varphi\left(-t_{l(\varphi)-1}\right) \cdot S(t)+\sum_{j=1}^{l(\varphi)-1}\left(\varphi\left(-t_{l(\varphi)-j-1}\right)-\varphi\left(-t_{l(\varphi)-j}\right)\right) \cdot S(t-2 j) .
$$

We observe that $\varphi$ is monotone in each of the intervals $\left(-\infty,-t_{l(\varphi)-1}\right],\left[-t_{l(\varphi)-1}\right.$, $\left.-t_{l(\varphi)-2}\right], \ldots,\left[-t_{1},-t_{0}\right],\left[-t_{0},+\infty\right)$. From Proposition 2.16 it follows that, for any $\varepsilon>0$, there exists an $A S^{1}(\mathbb{R})$ function $\varphi_{\varepsilon}$ and an orientation-preserving $C^{1}$ diffeomorphism $h: \mathbb{R} \rightarrow \mathbb{R}$ such that $V_{\varphi_{\varepsilon}-\varphi} \leq \varepsilon$ and $\varphi_{\varepsilon} \circ h=s \cdot \widehat{\varphi}$. Therefore,

$$
V_{s \cdot(\varphi \circ h)-\widehat{\varphi}}=V_{\varphi \circ h-s \cdot \widehat{\varphi}}=V_{\varphi \circ h-\varphi_{\varepsilon} \circ h}=V_{\varphi-\varphi_{\varepsilon}} \leq \varepsilon,
$$


proving the first claim. Furthermore, by the Bounding Lemma,

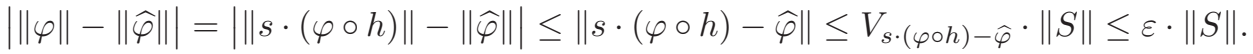

Thus, passing to the limit for $\varepsilon$ tending to 0 , also the second claim is proved.

\section{OPEN PROBLEMS AND CONCLUSIONS}

In this paper we have studied the main properties of the reparametrization invariant norms on $A S^{1}(\mathbb{R})$, focusing on the key role of standard RPI-norms. We have proved that these norms allow for the reconstruction of any piecewise monotone $C^{1}$-function with compact support up to reparametrization and sign, thus determining the value of any other RPI-norm on the same function.

However, many problems remain open. First of all, the theory has been developed just for the space $A S^{1}(\mathbb{R})$, and our last results also require the assumption that the considered functions are piecewise monotone and have compact support. Extensions to less regular spaces could be desirable, especially since the $C^{1}$ assumption makes our normed space not complete.

Moreover, we have left open the question about the existence of reparametrization invariant norms not obtainable as the sup of standard RPI-norms.

We postpone research on these issues to other papers.

\section{ACKNOWLEDGMENTS}

We wish to thank L. Ambrosio, N. Arcozzi, A. Baldi, A. Cohen, D. Guidetti, S. Masnou, D. Morbidelli, P. Papini and B. Volzone for their helpful suggestions. The authors are solely responsible for any possible errors. Thanks to M. Ferri for his indispensable support and friendship.

This paper is dedicated to Matteo, Marta and Tommaso.

\section{REFERENCES}

1. L. Ambrosio, N. Fusco, D. Pallara, Functions of bounded variation and free discontinuity problems, Oxford Mathematical Monographs, Oxford, 2000. MR.1857292(2003a:49002)

2. F. L. Bauer, J. Stoer and C. Witzgall, Absolute and monotonic norms, Numerische Mathematik, 3 no. 1 (1961), 257-264. MR0130104 (23:B3136)

3. J. Bergh, J. Löfström, Interpolation Spaces, Grundlehren der mathematischen Wissenschaften 223, 1976.

4. P. Donatini and P. Frosini, Natural pseudodistances between closed manifolds, Forum Mathematicum 16 (2004), 695-715. MR2096683 (2005g:58019)

5. P. Donatini and P. Frosini, Lower bounds for natural pseudodistances via size functions, Archives of Inequalities and Applications 2 (2004), 1-12. MR2043046 (2004m:58015)

6. P. Donatini and P. Frosini, Natural pseudodistances between closed surfaces, J. Eur. Math. Soc. (JEMS) 9 (2007), 231-253. MR2293959

7. A. Dumitrescu and G. Rote, On the Fréchet distance of a set of curves In: Proceedings of the 16th Canadian Conference on Computational Geometry (CCCG'04), Montreal, August 9-11, 2004, 162-165.

8. T. Holmstedt, J. Peetre, On certain functionals arising in the theory of interpolation spaces, J. Functional Anal. 4 (1969), 88-94. MR0241966 (39:3301)

9. K. Jarosz, Uniqueness of translation invariant norms, J. Funct. Anal. 174 (2000), no. 2, 417-429. MR 1768981 (2001e:46092)

10. P. W. Michor and D. Mumford, Riemannian geometries on spaces of plane curves, J. Eur. Math. Soc. (JEMS) 8 (2006), 1-48. MR2201275 (2007a:58007) 
11. E. Moreno and A. R. Villena, Uniqueness of dilation invariant norms, Proc. Amer. Math. Soc. 132 (2004), no. 7, 2067-2073. MR2053979 (2005b:46063)

12. B. Sévennec, Normes invariantes par diffomorphismes sur $C^{k}(X)$ (French) [Diffeomorphisminvariant norms on $C^{k}(X)$ ], Ann. Fac. Sci. Toulouse Math. (6) 7 (1998), no. 2, 335-355. MR $1656173(99 \mathrm{j}: 58022)$

Arces, Università di Bologna, via Toffano 2/2, I-40135 Bologna, Italia - And - Dipartimento di Matematica, Università di Bologna, P.zza di Porta S. Donato 5, I-40126 Bologna, Italia

E-mail address: frosini@dm.unibo.it

Dismi, Università di Modena e Reggio Emilia, via Amendola 2, Pad. Morselli, I-42100 RegGio Emilia, Italia 